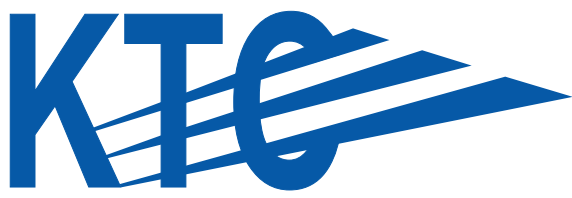

KENTUCKY TRANSPORTATION CENTER

\title{
GUIDELINES FOR ROAD DIET CONVERSIONS
}

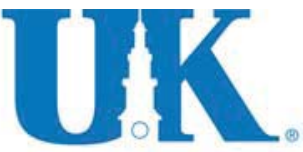

U N I V E R S I T Y O F

KENTUCKY

College of Engineering 


\section{K担}

\section{OUR MISSION}

We provide services to the transportation community through research, technology transfer and education.

We create and participate in partnerships

to promote safe and effective transportation systems.

\section{OUR VALUES}

\section{Teamwork}

Listening and communicating along with courtesy and respect for others.

\section{Honesty and Ethical Behavior}

Delivering the highest quality products and services.

\section{Continuous Improvement}

In all that we do. 
Research Report

KTC-11-19 / SPR415-11-1F

\title{
GUIDELINES FOR ROAD DIET CONVERSIONS
}

\section{Final Report}

\author{
by \\ Nikiforos Stamatiadis \\ Professor \\ Adam Kirk \\ Research Engineer \\ Chen Wang \\ Graduate Research Assistant \\ Andrea Cull \\ Graduate Research Assistant \\ And \\ Nithin Agarwal \\ Graduate Research Assistant \\ Department of Civil Engineering \\ and \\ Kentucky Transportation Center \\ College of Engineering \\ University of Kentucky \\ Lexington, Kentucky
}

The contents of this report reflect the views of the authors who are responsible for the facts and accuracy of the data presented herein. The contents do not necessarily reflect the official views of policies of the University of Kentucky, the Kentucky Transportation Cabinet, or the Federal Highway Administration. This report does not constitute a standard, specification, or regulation. The inclusion of manufacture names and trade names is for identification purposes and is not to be considered an endorsement.

November 2011 


\begin{tabular}{|c|c|c|c|c|}
\hline $\begin{array}{l}\text { 1. Report Number } \\
\text { KTC-11-19 / SPR415-11-1F }\end{array}$ & \multicolumn{2}{|c|}{ 2. Government Accession No. } & \multicolumn{2}{|c|}{ 3. Recipient's Catalog No. } \\
\hline \multirow{2}{*}{\multicolumn{3}{|c|}{$\begin{array}{l}\text { 4. Title and Subtitle } \\
\text { Guidelines for Road Diet Conversions }\end{array}$}} & \multicolumn{2}{|l|}{$\begin{array}{l}\text { 5. Report Date } \\
\text { November } 2011\end{array}$} \\
\hline & & & \multicolumn{2}{|c|}{ 6. Performing Organization Code } \\
\hline \multicolumn{3}{|c|}{$\begin{array}{l}\text { 7. Author(s) } \\
\text { N. Stamatiadis, A. Kirk, C. Wang, and A. Cull }\end{array}$} & \multicolumn{2}{|c|}{$\begin{array}{l}\text { 8. Performing Organization Report No. } \\
\text { KTC-11-19 / SPR415-11-1F }\end{array}$} \\
\hline \multirow{2}{*}{\multicolumn{3}{|c|}{$\begin{array}{l}\text { 9. Performing Organization Name and Address } \\
\text { Kentucky Transportation Center } \\
\text { College of Engineering } \\
\text { University of Kentucky } \\
\text { Lexington, Kentucky 40506-0281 }\end{array}$}} & \multicolumn{2}{|l|}{ 10. Work Unit No. } \\
\hline & & & \multicolumn{2}{|c|}{$\begin{array}{l}\text { 11. Contract or Grant No. } \\
\text { SPR316 }\end{array}$} \\
\hline \multirow{2}{*}{\multicolumn{3}{|c|}{$\begin{array}{l}\text { 12. Sponsoring Agency Name and Address } \\
\text { Kentucky Transportation Cabinet } \\
200 \text { Mero Street } \\
\text { Frankfort, KY }\end{array}$}} & \multicolumn{2}{|c|}{$\begin{array}{l}\text { 13. Type of Report and Period Covered: } \\
\text { Final Report; } 07 / 10-12 / 11\end{array}$} \\
\hline & & & \multicolumn{2}{|c|}{ 14. Sponsoring Agency Code } \\
\hline \multicolumn{5}{|l|}{ 15. Supplementary Notes } \\
\hline \multicolumn{5}{|c|}{$\begin{array}{l}\text { 16. Abstract } \\
\text { The purpose of this report is to document the development of the guidelines for the road diet } \\
\text { conversions prepared for the Kentucky Transportation Cabinet. The guidelines consider operational } \\
\text { and safety aspects of the conversions to assist in the preliminary determination of whether a road diet } \\
\text { conversion is appropriate for a given roadway segment. The guidelines provide suggested cross } \\
\text { section designs, transition to and from the road diet section and a flow chart for determining the } \\
\text { appropriate actions. }\end{array}$} \\
\hline \multicolumn{2}{|c|}{$\begin{array}{l}\text { 17. Key Words } \\
\text { Intersection Design, Roundabout, Inside left-turn, median } \\
\text { u-turn, superstreet, continuous flow, jughandle, bowtie, } \\
\text { intersection evaluation }\end{array}$} & \multicolumn{3}{|c|}{$\begin{array}{l}\text { 18. Distribution Statement } \\
\text { Unlimited }\end{array}$} \\
\hline $\begin{array}{l}\text { 19. Security Classification (report) } \\
\text { Unclassified }\end{array}$ & $\begin{array}{l}\text { 20. Security Clas } \\
\text { page) Unclas }\end{array}$ & $\begin{array}{l}\text { fication (this } \\
\text { ified }\end{array}$ & 21. No. of Pg: 94 & 22. Price $\$ 0$ \\
\hline
\end{tabular}




\section{TABLE OF CONTENTS}

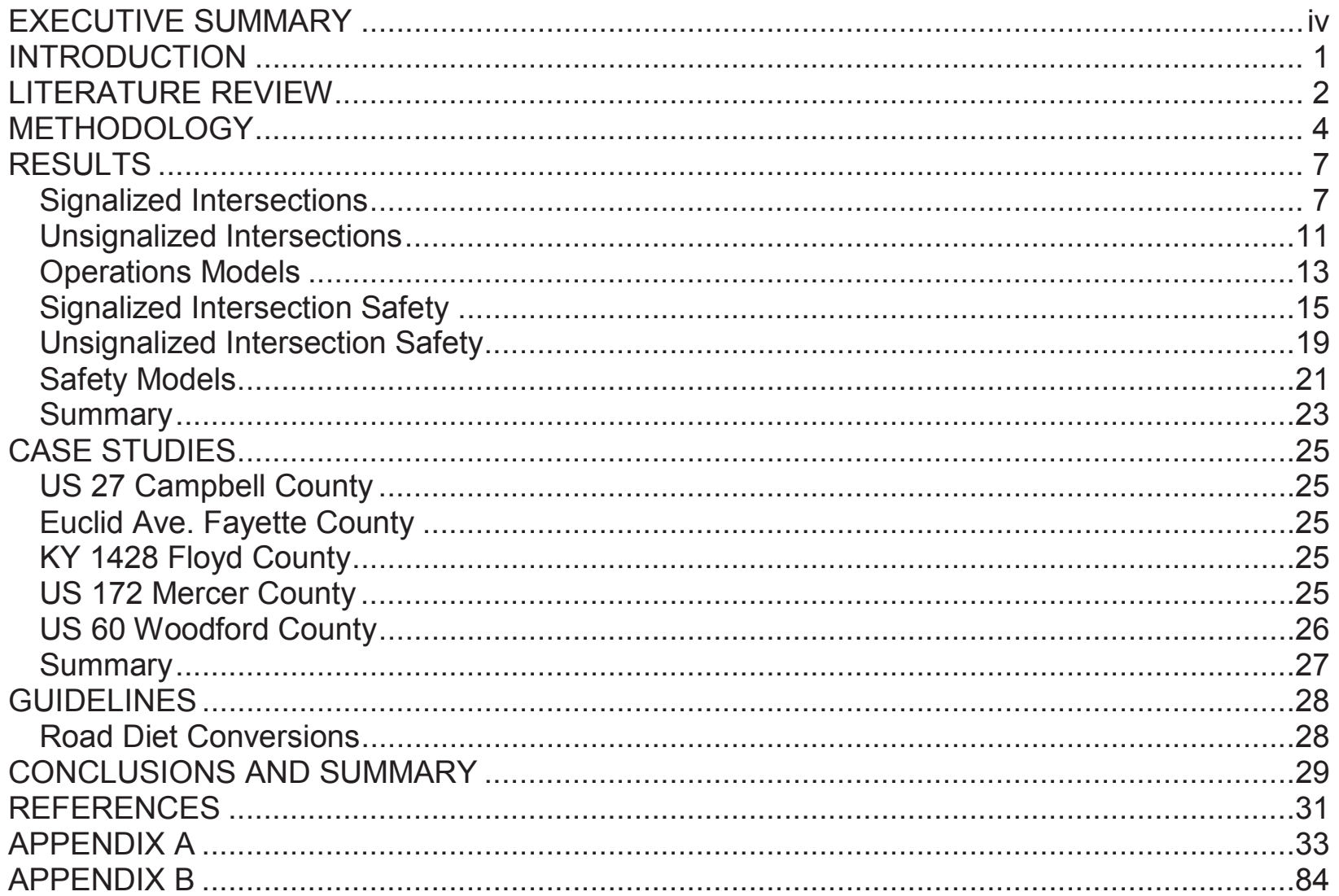

\section{LIST OF FIGURES}

Figure 1 Signalized intersection delay differences (3-4), 300 vph side street volume ............... 7 Figure 2 Signalized intersection delay differences (3-4), 700 vph side street volume .............. 8 Figure 3 Signalized intersection delay differences (3-4), 1,300 vph side street volume ............ 9 Figure 4 Signalized intersection average queue differences (3-4), 300 vph side street volume .10 Figure 5 Signalized intersection average queue differences (3-4), 700 vph side street volume 10 Figure 6 Signalized intersection average queue differences (3-4), 1,300 vph side street volume

Figure 7 Unsignalized intersection side street delay differences (3-4), $300 \mathrm{vph}$ side street

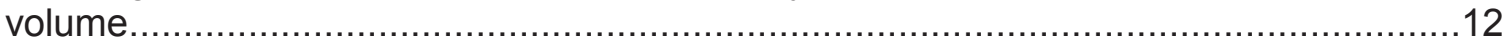

Figure 8 Unsignalized intersection side-street delay differences (3-4), $700 \mathrm{vph}$ side street volume.

Figure 9 Guideline for operational performance at signalized intersections ..........................14

Figure 10 Difference in main street rear end conflicts between four- and three-lane signalized intersections (3-4), by side street volume, 5 percent left turns .................................16

Figure 11 Difference in main street rear end conflicts between four- and three-lane signalized intersections (3-4), by side street volume, 20 percent left turns ...............................17

Figure 12 Difference in main street rear end conflicts between four- and three-lane signalized intersections (3-4), by side street volume, 35 percent left turns ................................18

Figure 13 Difference in main street lane change conflicts between four- and three-lane signalized intersections (3-4), 700 vph side street volume. 19 
Figure 14 Difference in main street rear end conflicts between four- and three-lane unsignalized intersections (3-4), 700 vph side street volume........................................................20

Figure 15 Difference in main street lane change conflicts between four- and three-lane signalized intersections (3-4), $700 \mathrm{vph}$ side street volume....

Figure 16 Guidelines for rear end conflicts by main and side street volume and percent left-turns

Figure 17 Guidelines for lane change conflicts by major/minor street volumes and percent left17 Guidelines for lane change conflicts by major/minor street volumes and percent left-

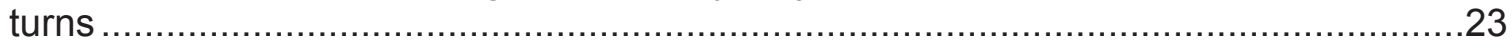

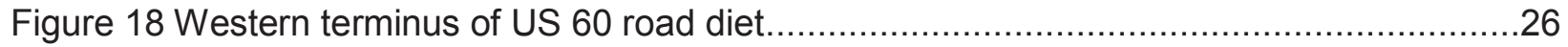

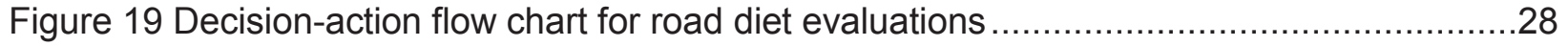

\section{LIST OF TABLES}

Table 1 Level of service and maximum sum of critical lane volumes at signalized intersections.. 4

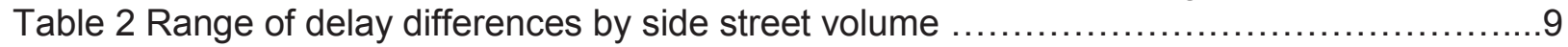

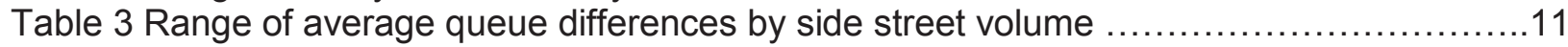

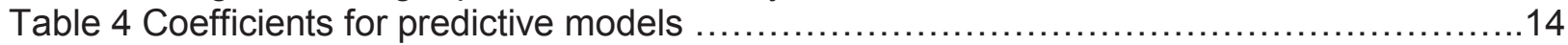

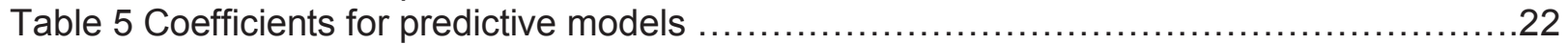




\section{TABLE OF CONTENTS}

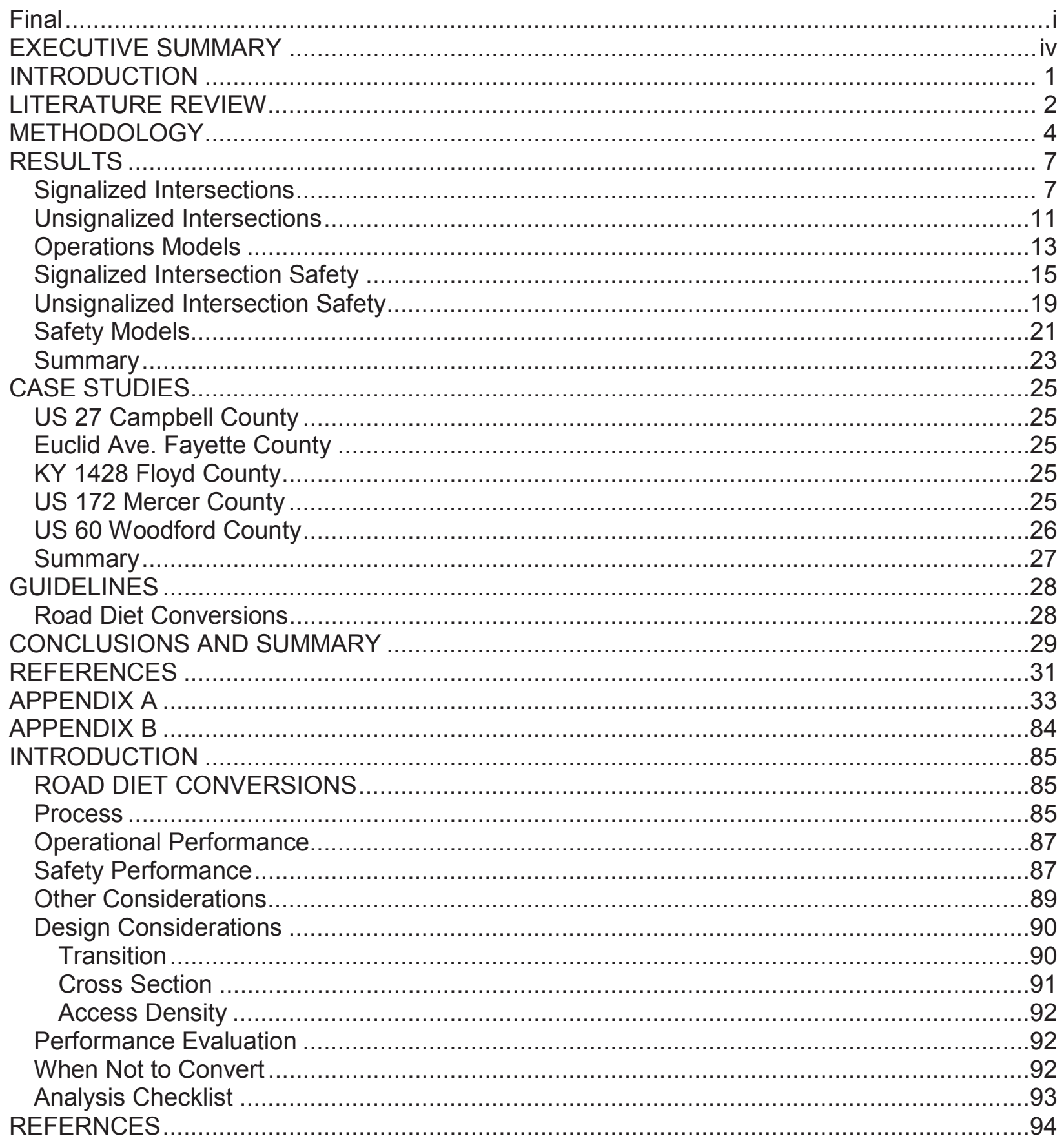

\section{LIST OF FIGURES}

Figure 1 Signalized intersection delay differences (3-4), 300 vph side street volume ............... 7

Figure 2 Signalized intersection delay differences (3-4), 700 vph side street volume ............... 8

Figure 3 Signalized intersection delay differences (3-4), 1,300 vph side street volume ............ 9

Figure 4 Signalized intersection average queue differences (3-4), 300 vph side street volume .10

Figure 5 Signalized intersection average queue differences (3-4), 700 vph side street volume 10 
Figure 6 Signalized intersection average queue differences (3-4), 1,300 vph side street volume 11

Figure 7 Unsignalized intersection side street delay differences (3-4), $300 \mathrm{vph}$ side street volume

Figure 8 Unsignalized intersection side-street delay differences (3-4), $700 \mathrm{vph}$ side street volume....

Figure 9 Guideline for operational performance at signalized intersections

Figure 10 Difference in main street rear end conflicts between four- and three-lane signalized intersections (3-4), by side street volume, 5 percent left turns

Figure 11 Difference in main street rear end conflicts between four- and three-lane signalized intersections (3-4), by side street volume, 20 percent left turns

Figure 12 Difference in main street rear end conflicts between four- and three-lane signalized intersections (3-4), by side street volume, 35 percent left turns ................................18

Figure 13 Difference in main street lane change conflicts between four- and three-lane signalized intersections (3-4), 700 vph side street volume....

Figure 14 Difference in main street rear end conflicts between four- and three-lane unsignalized intersections (3-4), 700 vph side street volume.

Figure 15 Difference in main street lane change conflicts between four- and three-lane signalized intersections (3-4), 700 vph side street volume.

Figure 16 Guidelines for rear end conflicts by main and side street volume and percent left-turns

Figure 17 Guidelines for lane change conflicts by major/minor street volumes and percent left-

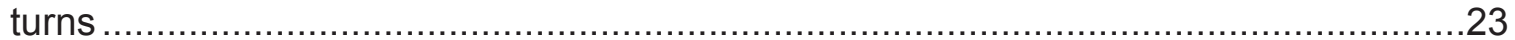

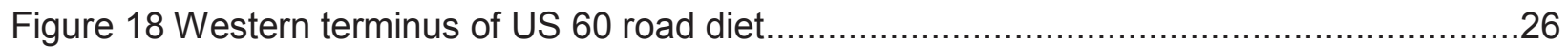

Figure 19 Decision-action flow chart for road diet evaluations .........................................28

\section{LIST OF TABLES}

Table 1 Level of service and maximum sum of critical lane volumes at signalized intersections.. 4

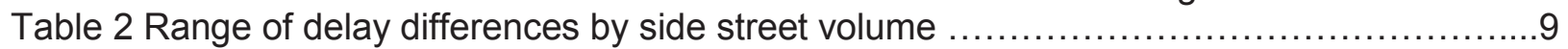

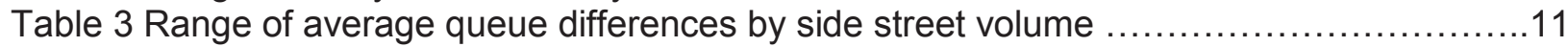

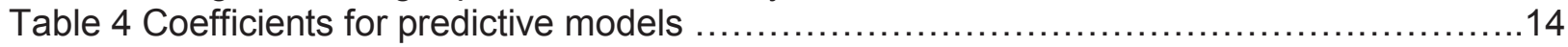

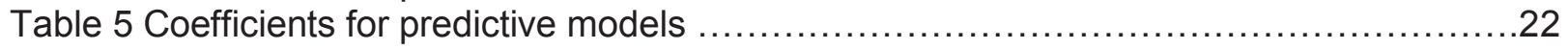




\section{TABLE OF CONTENTS}

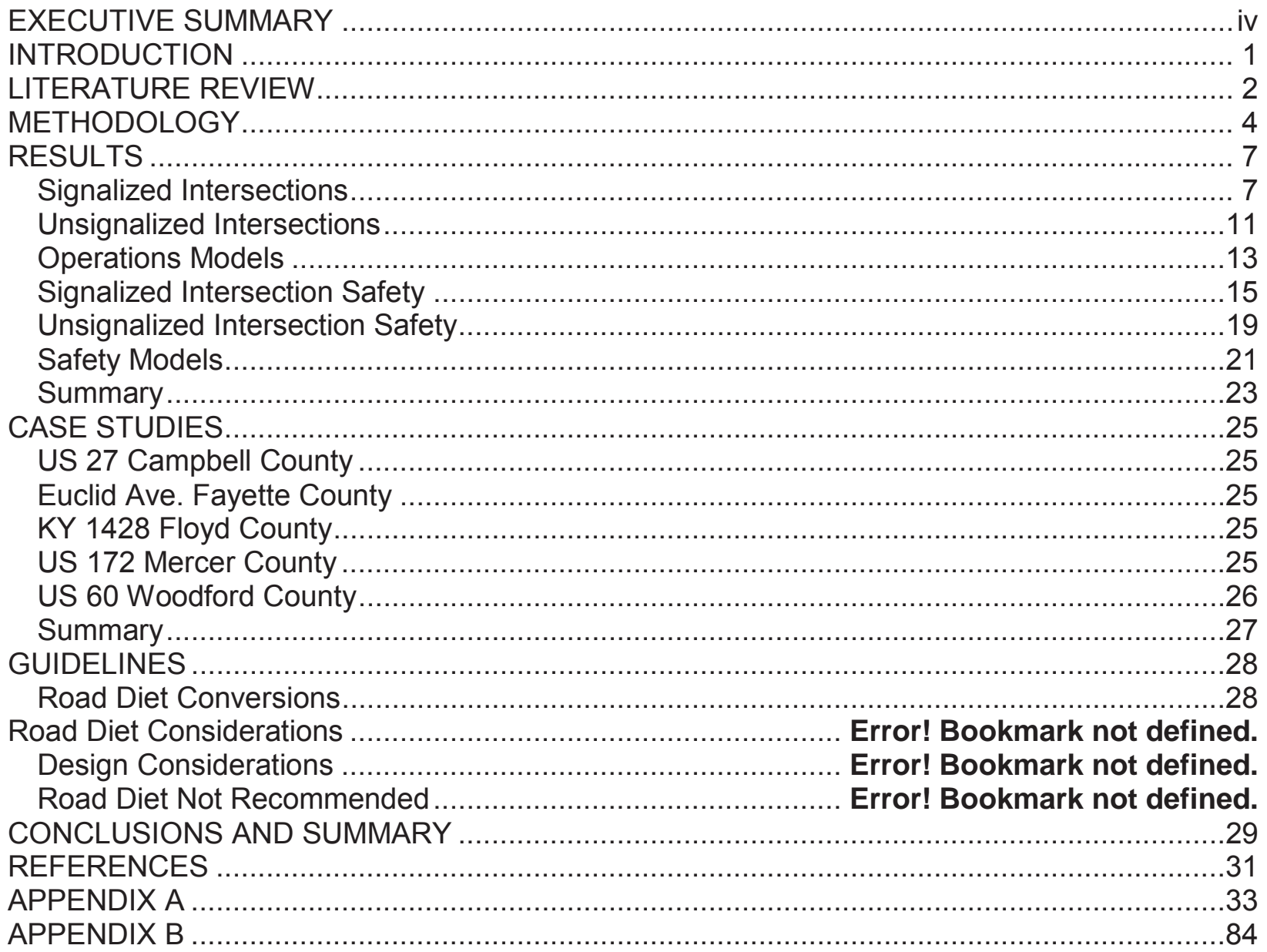

\section{LIST OF FIGURES}

Figure 1 Signalized intersection delay differences (3-4), 300 vph side street volume .............. 7 Figure 2 Signalized intersection delay differences (3-4), 700 vph side street volume ............... 8 Figure 3 Signalized intersection delay differences (3-4), 1,300 vph side street volume ............ 9 Figure 4 Signalized intersection average queue differences (3-4), 300 vph side street volume .10 Figure 5 Signalized intersection average queue differences (3-4), 700 vph side street volume 10 Figure 6 Signalized intersection average queue differences (3-4), 1,300 vph side street volume

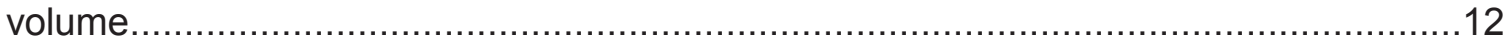

Figure 8 Unsignalized intersection side-street delay differences (3-4), $700 \mathrm{vph}$ side street

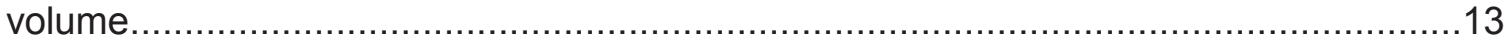

Figure 9 Guideline for operational performance at signalized intersections ...........................14

Figure 10 Difference in main street rear end conflicts between four- and three-lane signalized intersections (3-4), by side street volume, 5 percent left turns ...............................16

Figure 11 Difference in main street rear end conflicts between four- and three-lane signalized intersections (3-4), by side street volume, 20 percent left turns 
Figure 12 Difference in main street rear end conflicts between four- and three-lane signalized intersections (3-4), by side street volume, 35 percent left turns ..................................18

Figure 13 Difference in main street lane change conflicts between four- and three-lane signalized intersections (3-4), 700 vph side street volume.

Figure 14 Difference in main street rear end conflicts between four- and three-lane unsignalized

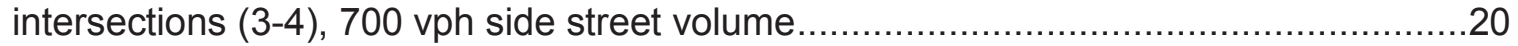

Figure 15 Difference in main street lane change conflicts between four- and three-lane signalized intersections (3-4), 700 vph side street volume. 21

Figure 16 Guidelines for rear end conflicts by main and side street volume and percent left-turns

Figure 17 Guidelines for lane change conflicts by major/minor street volumes and percent left-

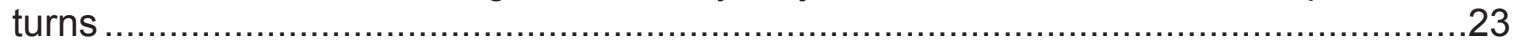

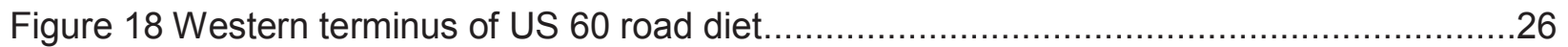

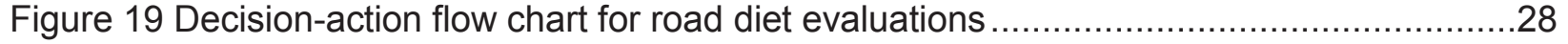

\section{LIST OF TABLES}

Table 1 Level of service and maximum sum of critical lane volumes at signalized intersections..4

Table 2 Range of delay differences by side street volume $\ldots \ldots \ldots \ldots \ldots \ldots \ldots \ldots \ldots \ldots \ldots \ldots \ldots$

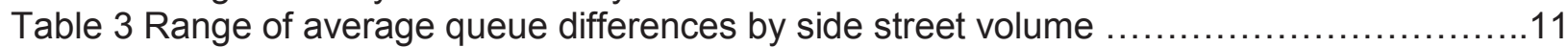

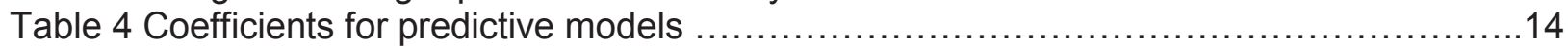

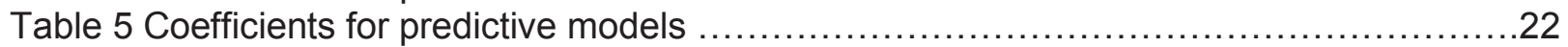




\section{EXECUTIVE SUMMARY}

The need for innovative solutions in addressing mobility and safety concerns in an environment with budgetary constraints is paramount. Road diets are one such innovative solution, seeking to develop multimodal corridors while retaining the original right of way. Road diets usually involve restriping a four-lane undivided road as a three-lane road with two through lanes and a two-way left-turn lane. The extra roadway width can be converted to widen existing lanes, create bicycle lanes, supply on street parking, widen sidewalks, or provide opportunities for landscaped median islands. The main focus of past research on road diets has been on evaluating their safety and very little exists in providing design guidance on when such conversions will work. This report provides such guidance in determining the appropriateness of road diet conversions and identified parameters to be considered during such evaluations.

Typically, road diet conversions will operate at acceptable levels as long as the signalized intersections do not present any operational problems. The development of the guidelines analysis focused on evaluating and comparing the operation of three- and four-lane roads at signalized intersections. The critical aspect in this approach is to determine the scenarios and provide basic guidance as to when the road diet conversion is appropriate. The research team used methodologies that could be used to directly link the traffic demand, i.e., design hour volumes and turning movement volumes, to the comparative operation of the two designs. A series of simulations were undertaken to establish the required guidelines for road diet conversions. These simulations were performed with the CORridor SIMulation (CORSIM) and VISSIM software as well as the Safety Surrogate Assessment Model (SSAM).

An important aspect of the work completed here is the extension of the usable range of volumes where road diets could be beneficial. Past work recommended their application for roads with an ADT up to 17,000 vehicles per day (vpd). The current research indicates that such conversions could work for roads with significantly greater volumes, up to 23,000 vpd. Moreover, the findings here identify the effect of the side street volume, indicating that both volumes need to be considered when determining whether such a conversion should be considered.

The simulation results from CORSIM support the assumption that a four-lane option will have a critical lane volume lower than that of a three-lane alternative. This could indicate an operational advantage of the four-lane option when compared to that of the three-lane. For signalized intersections, there are certain conditions where the road diet could be the preferred option over a four-lane alternative. The data also indicates that there is an effect of the side street volume in determining these conditions and that the combination of main and side street volumes needs to be considered in determining whether the road diet is appropriate. Another aspect that could be of interest regarding the operational efficiency of road diets is the resulting queue lengths. An evaluation was completed for this aspect using the same data. The analysis showed that in general the road diet produced smaller or the same average queue lengths than the four-lane roads.

A similar analysis for unsignalized intersections showed that all delays along the major road were very small and the three-lane option had slightly higher delays than the four-lane option. However, these differences were very small and most were less than $2.5 \mathrm{sec} / \mathrm{veh}$ with the majority (56 percent of the cases) of the delay differences being $1 \mathrm{sec} / \mathrm{veh}$ or less. The significant differences in the unsignalized intersections were noted for the delays along the side street. For all scenarios tested, the delays on the side street for a road diet conversion were smaller than those observed for the four-lane road. 
An analysis of the safety aspects of road diets was also conducted using the simulation models. It should be noted that the analysis presented uses a surrogate measure for safety, i.e. potential number of conflicts, and not crashes. The simulation results showed that, in general, the number of rear end conflicts at the signalized intersections will increase with a road diet, when left-turn percentages are low, and will decrease with a road diet when left-turn percentages are high. This was an expected result, since the left-turn vehicles can block the through lane increasing the probability of rear end conflicts.

The analysis for the lane change conflicts showed that at low volumes neither the 3-lane or 4lane alternative had a high number of conflicts, and the road diet produced less lane change conflicts at higher volumes. This was an expected result, since the number of lanes is reduced and thus the opportunity for such conflicts is also reduced. Left-turn percentage was not identified as a significant predictor for lane change conflicts.

Angle crashes were not modeled using conflict analysis due to software limitations, but case studies evaluated in Kentucky and presented below indicate a general reduction in angle crashes as the result of road diets.

Four road diet conversions in Kentucky were identified and evaluated to determine their overall effects and identify elements that could also assist in the development of the guidelines. In general, it was concluded that the four cases evaluated are successful road diet conversions. The installations covered roadway lengths between 0.82 to 1.41 miles and a variety of conditions. The ADT range of 7,400 to 13,700 vehicles per day also indicates a wide range of volumes where road diets have been implemented. In three of the four cases, safety improved significantly and sideswipe and rear end crashes showed large reductions. There were no significant congestion issues resulting from the installation, and traffic volumes showed a slight decrease after the conversion. One of the cases that showed an increase in crashes after the installation points to the need for developing proper designs for the transition into and out of the road diet. This is critical to avoid potential safety problems associated with merging of traffic and thus creating an inappropriate environment. The same case also identifies access management as an issue for review to avoid potential safety problems.

A set of guidelines was developed using the analysis completed here. One of the important findings of this research is the expansion of the usable range for road diets. Prior experience has limited their application to roadways with an ADT of 17,000 vehicles per day. This research identifies the importance of side street volumes and supports the utilization of road diets on roadways with volumes up to 23,000 vehicles per day. A flow chart identifying required steps to be undertaken to evaluate whether a road diet conversion is appropriate is also provided as part of the guidelines. Problems correctable with a road diet are also identified and a list of required elements to be studied for an in-depth evaluation of road diet installation is also presented. 


\section{INTRODUCTION}

Rural and urban roadways are becoming increasingly congested throughout the US and other countries and solutions are sought that could facilitate mobility along these facilities. A typical approach for solving this problem has been the addition of lanes but this approach has limited success. At the same time, roadway widening is a very expensive and environmentally disruptive practice that frequently offers only short term relief. The need for innovative solutions in addressing mobility and safety concerns in an environment with budgetary constraints is paramount. Such innovative solutions seek to develop multimodal corridors while retaining the original right of way and among them is the concept of road diet, where the number of travel lanes is reduced. Road diets usually involve restriping a four-lane undivided road as a threelane road with two through lanes and a two-way left-turn lane. The extra roadway width can be converted to widen existing lanes, create bicycle lanes, supply on street parking, widen sidewalks, or provide opportunities for landscaped median islands.

The road diet approach is a relatively new concept that may improve mobility on a roadway without requiring additional right-of-way. Often the inside through lane on a four-lane undivided roadway acts like a de facto turn-lane, diminishing through capacity. The introduction of a twoway left-turn lane (TWLTL) can often meet the left-turn demand for both directions of travel. Therefore, road diets are a design tool that can be used within existing right of way at a very low cost to improve mobility, and they frequently have no or few negative impacts.

Recently completed studies throughout the US indicate that this alternative is successful in improving both safety and operations along corridors. Such conversions are often considered when there are a significant number of crashes associated with a large number of left-turning vehicles with no turning lanes present. These conversions not only improve the mobility for vehicles, but for non-motorized users (pedestrians and bicyclists) as well, and they also enhance their safety.

Past research has focused on evaluating mainly the safety of road diets. However, there is practically no literature providing guidance on the details of the designs or information as to when such conversions will work. The need to identify criteria to be considered for establishing road diets is critical and should be addressed. This report provides such guidance in determining the appropriateness of road diet conversions and identified parameters to be considered during such evaluations. 


\section{LITERATURE REVIEW}

Past research has primarily focused on evaluating the safety of road diets. However, there is practically no literature providing guidance on the details of the geometric designs or information as to when such conversions will work from an operational standpoint. A review of all State DOT design manuals and guides did not reveal any guidance regarding road diet conversions.

Road diets can improve operating efficiency and safety for all users. The operational efficiency is the result of removing left-turning traffic and thus avoiding queues behind such vehicles while at the same time lane changes are eliminated (due to the single through lane). The potential for lower operating speeds typically exists in such conversions but this could be viewed as beneficial for the safety of the segment, resulting in lower severity crashes and providing longer reaction time (Huang et al. 2002). The safety of other users could also improve. Bicyclists can have a separate lane which typically improves safety and pedestrians have shorter widths to cross.

A more recent study in lowa evaluated crash data for 30 sites (15 road diets and 15 comparison sites) over a 23 year period (Pawlovich et al 2006). The study concluded that, after conversion, there was a 25.2 percent reduction in crash frequency and an 18.8 percent reduction in crash rate. Another study using Minnesota road diet conversions also supported safety gains for such roadways (Gates et al 2007). Their study showed reductions of approximately 44 percent in the total number of crashes. In addition, the results showed reductions for injury crashes (45.7 percent) and right angle crashes (37 percent). Reductions were also noted for rear end and leftturn crashes but these reductions were not statistically significant. The study also evaluated before-and-after speeds showing reductions both in mean and $85^{\text {th }}$-percentile speeds after the roadway conversion.

A study evaluated recent road diet conversions and developed crash modification factors (CMF) for such conversions (FHWA 2010). The study identifies basic benefits of road diets, such as speed reduction, improved safety, and reduced vehicle-pedestrian conflicts. A main goal of the study was to update an earlier study that documented an 18.8 percent reduction in crash rates for road diet conversions (FHWA 2004). The study used a Bayesian approach to compare untreated sites to those where a road diet was implemented. The results clearly indicate that there are safety benefits from these conversions and the magnitude of the benefits depends on the characteristics of the site. The study found an average of 29 percent reduction in total crashes for the treated sites which was attributed to the road diet. Other results include an average reduction of $5 \mathrm{mph}$ in the average speed of motorists and a decrease in percent of drivers driving over $5 \mathrm{mph}$ above the speed limit. The study recommends a CMF of 0.71 for road diet conversions indicating a 29 percent reduction in overall crashes after a road diet installation.

Road diets have been used on roads with an average daily traffic (ADT) of up to 25,000 (Burden and Lagerwey 1999). Other studies have also indicated that capacity is not affected by the elimination of the lane and often no increase in congestion is observed (Welch 1999). An issue of some concern is the potential for diverting traffic to other streets parallel to the converted facility, and this is an aspect that should be monitored over time when evaluating such an option (Rosales 2006).

Improvements in livability conditions and associated benefits are elements to be considered during road diet conversions, as review of past case studies has indicated (Rosales and Knapp 2005). The reduction of the roadway width will make it easier for pedestrians to cross the road 
at both signalized and unsignalized intersections, increase feeling of a "safer and more comfortable" street, encourage an increase in pedestrian and bicyclist traffic, and encourage economic growth and redevelopment at a quicker pace (Rosales and Knapp 2005).

The first attempt in defining guidelines for road diet conversions was completed in 2001 (Knapp and Giese 2001). This effort relied on evaluating before-after conditions on existing road diet projects completed at that time and documented several of the benefits from such conversions. The case studies reviewed showed that the $85^{\text {th }}$-percentile speeds were in general reduced by less than five miles per hour and there was a significant decrease for speeds above the posted speed limit. The review also identified safety improvements with total crash reductions between 17 to 62 percent. The 13 roadway conversions reviewed had ADT volumes of 8,400 to 24,000 vehicles per day (vpd). Simulation was also used to identify potential factors that could be used in determining whether a road diet conversion would be appropriate. The study recommended that a road diet conversion could be considered feasible for roads with an ADT between 15,000 and 17,500 vpd. The authors also emphasized that individual detailed analysis is required once such conversions are considered feasible in order to evaluate the corridor conditions and details, such as access frequency and left-turn percentages. The study also identified a list of elements that need to be considered during the evaluation process.

A more recent attempt to improve on the prior guidelines for road diets was completed in 2006 (Rosales 2006). The main effort was to assess exiting road diets and identify the livability benefits from such roadway conversions considering improved mobility for all users and enhanced street character. The resulting effort developed a step by step process in planning, analysis, and implementation of road diet projects and provided a guide for decision-makers. However, the processes developed remained very general in nature and did not provide specific guidance regarding volumes or left-turn percentages indicating when such a project could result in improved operational and safety conditions.

The review concluded that the literature is inadequate with respect to providing guidance on implementing road diets. The need to identify criteria to be considered for establishing road diets is critical and should be addressed. Issues regarding geometric features of the road to be converted and operational elements to be analyzed should be identified. 


\section{METHODOLOGY}

Typically, road diet conversions will operate at acceptable levels as long as the signalized intersections do not present any operational problems (Welch 1999). Therefore, this analysis focused on evaluating and comparing the operation of three- and four-lane roads at signalized intersections. The critical aspect in this approach is to determine the scenarios and provide basic guidance as to when the road diet conversion is appropriate. The research team used methodologies that could be used to directly link the traffic demand, i.e. turning movement volumes, to the comparative operation of the two designs.

The most promising approach that could be used here for evaluating and comparing the two alternatives was determined to be Critical Lane Analysis (CLA). Critical Lane Analysis, as developed by Messer and Fambro (1977), uses the geometry of the intersection along with intersection traffic volumes as the basis for establishing a measure of potential performance and, by extent, of capacity. The method apportions traffic volumes to each of the available lanes and considers the phasing plans that could be appropriate to accommodate the intersection movements. A critical volume (i.e. heaviest lane volume or combination of lane volumes) for each phase is calculated and the sum of these volumes is the total critical volume for the intersection. This sum can then be directly related to the level of service definition for signalized intersections (Table 1). This methodology establishes the capacity of the intersection based on the volume of conflicting flows for different phasing options and geometry. In general, a critical sum of 1,400 vehicles per hour (vph) is assumed to allow for under capacity operation for a signalized intersection.

Table 1 Level of service and maximum sum of critical lane volumes at signalized intersections

\begin{tabular}{cccccc}
\hline Level of & Traffic Flow & Volume to & \multicolumn{3}{c}{ Critical Lane Volumes (vph) } \\
\cline { 4 - 6 } Service & Condition & Capacity Ratio & Two-Phase & Three-Phase & Multiphase \\
\hline A & Stable & $<.6$ & 900 & 855 & 825 \\
B & Stable & $<.7$ & 1050 & 1000 & 965 \\
C & Stable & $<.8$ & 1200 & 1140 & 1100 \\
D & Unstable & $<.85$ & 1275 & 1200 & 1175 \\
E & Capacity & $<1.0$ & 1500 & 1425 & 1375 \\
\hline
\end{tabular}

Source: Messer and Fambro, 1977

A series of simulations were undertaken to establish the required guidelines for road diet conversions. These simulations were performed with the CORridor SIMulation (CORSIM) and VISSIM software and were also evaluated using the Safety Surrogate Assessment Model (SSAM).

The literature identifies that road diets have been implemented for roadways with ADT as high as 24,000 vehicles per day (vpd). However, in Kentucky most of the existing conversions range between 7,400 to $13,700 \mathrm{vpd}$. Preliminary capacity analysis indicated a theoretical maximum capacity of 2,400 vph on a three-lane section. Therefore, this was used to set the upper threshold of evaluated traffic volumes. The $2,400 \mathrm{vph}$ threshold relates to an ADT of approximately 24,000 ADT assuming a $\mathrm{K}$ factor of 10 percent. ADTs ranging from 6,000 to $24,000 \mathrm{vpd}$ were evaluated in the study using an increment of 2,000 vpd. One directional split (50/50) was utilized. While 24,000 ADT was set as the maximum, these volumes produced unreliable and scattered results indicative of oversaturated conditions. Therefore, results and trends are only developed based on a maximum of 22,000 vpd or lower depending on side street volumes and signalization. 
Another aspect that could influence operations is the percentage of left-turns, since large numbers of left-turning vehicles have the potential to block traffic and cause the inside through lanes to operate as exclusive (de facto) left-turn lanes. A series of left-turn percentages were used ranging between 5 to 40 percent with increments of 5 percent. Finally, the percent of right turns used was set at 15 without any exclusive right turn lanes or bays. These values represent average operating parameters and are reflective of the majority of roadways.

The volumes of the cross streets were also varied to reflect possible trends and effects. These volumes varied between 3,000 to $13,000 \mathrm{vpd}$ with $2,000 \mathrm{vpd}$ increments. There was only one directional split (50/50) and a 10 percent estimate of left and right turns was used.

For unsignalized intersections, the main street volume was limited to 1,600 vph, since this would be the highest volume when the intersection will not require signalization based on Warrant 3 Peak Hour Volume (FHWA 2009). The same warrant also limits the maximum side street volume to $300 \mathrm{vph}$. Additional simulations were conducted for lower side street volumes of 100 vph and $200 \mathrm{vph}$ to allow for a complete evaluation of the unisgnalized intersection changes.

A total of 480 combinations were used for simulating signalized intersections, i.e. 10 volume scenarios for eight left-turn percentages and six cross street volumes. For unsignalized intersections, the total number of simulations was 144 , i.e. six volume scenarios for eight leftturn percentages and three cross street volumes.

An issue to be noted here is the fact that at certain times a four-lane roadway may operate as a three-lane road because the inside lanes will be required to accommodate significant left-turn volumes and act as left-turn lanes (a de facto left-turn lane). In this case, both the three and four-lane roads will produce the same critical volume for the main street, since this is the sum of the through traffic (allocated in one lane in both cases) and the left-turn traffic (also allocated in one lane). The presence of shared left and through lanes requires the use of left-turn equivalents (TRB 1985). These equivalents can then be used to define the point when the lane operations on a four-lane road shift from a shared lane to a de facto left-turn lane. This concept is demonstrated below, assuming a 50/50 split on the main street and equal percentages of leftturns. The critical volume for the four-lane option, $\mathrm{CV}_{4}$, and the three-lane, $\mathrm{CV}_{3}$, will be

$$
\begin{aligned}
& \mathrm{CV}_{4}=\mathrm{LT}+(\mathrm{LT}(\mathrm{LTE})+\mathrm{TH}) / 2 \text { and } \\
& \mathrm{CV}_{3}=\mathrm{LT}+\mathrm{TH} \\
& \text { where } \mathrm{LT}=\text { left-turn volume, } \\
& \mathrm{TH}=\text { through volume } \\
& \mathrm{LTE}=\text { left-turn equivalency factor. }
\end{aligned}
$$

Setting $\mathrm{CV}_{4}$ equal to $\mathrm{CV}_{3}$ derives the equation:

$$
L T+(L T(L T E)+T H) / 2=L T+T H \Rightarrow L T(L T E)=T H
$$

and since the total volume $V=L T+T H$, then $V=L T(L T E+1)$. In this case, if the $L T E$ is two, then the conversion will occur when left-turns exceed 33 percent; if the LTE is three then this will occur at 25 percent of left-turns; and if the LTE is four then this will occur at 20 percent of leftturns. The LTE is determined based on the magnitude of the opposing traffic, since it is assumed that left turns will block through traffic while waiting to complete the turn in the available gaps. Therefore, greater opposing volumes will result in fewer gaps and thus greater LTEs. It should be noted here that in these calculations, the critical volume of the four-lane road 
will always be lower than that of the three lanes, since the through traffic is distributed in more than one lane until the inside lane becomes a de facto left-turn. This analysis demonstrates that at low left-turn percentages, the four-lane roadway will operate more efficiently than a three-lane roadway, but at high percentages of left-turns there would be no fundamental difference in their operation.

In addition to the operational analysis, an evaluation of intersection safety was also completed using VISSIM and SSAM to determine the potential number of conflicts between the two options. SSAM is a tool developed by the Federal Highway Administration which analyzes vehicle trajectory output from the VISSIM micro-simulation model. SSAM identifies "conflicts" between vehicles, which are defined as instances of near misses between two vehicles. SSAM is capable of categorizing the conflicts as either rear-end or crossing angle and lane changing (sideswipe) crashes. It should be noted here that the estimate of conflicts is considered a safety surrogate measure and does reflect the actual number of crashes at an intersection or on a roadway segment. The number of conflicts is used to indicate potential relative changes in specific types of conflicts that could result in a crash. Two major categories are provided which include rear end and lane change conflicts. Lane change conflicts can lead to sideswipe crashes. Each potential conflict type was evaluated alone to determine the differential impact it could have on the safety performance of the two options.

The data obtained from both operational and safety analyses were used to develop predictive models that could be used in establishing potential guidelines for the implementation of road diets. The Statistical Package for the Social Sciences (SPSS) was utilized to develop these models. 


\section{RESULTS}

\section{Signalized Intersections}

The simulation results from CORSIM support the assumption that a four-lane option will have a critical lane volume lower than that of a three-lane alternative. This could indicate an operational advantage of the four-lane option when compared to that of the three-lane. These results are discussed below by examining the delay difference between the three- and four-lane options for a signalized intersection. The difference is calculated as the delay of the three-lane minus that of four-lane option. Therefore, a negative number indicates that the three-lane option has a lower delay than the four-lane option for the specific volume combination. Another issue to be noted in the analysis is that the peak hour volume used is the bidirectional traffic for the street.

The delay differences for the $300 \mathrm{vph}$ side street volume show different trends for each percent of left-turns evaluated (Figure 1). For low left-turn percentages, the trend shows that at low volumes the three-lane option is "better" than the four-lane (i.e. negative delay differences) and as the volumes increase the delay differences become smaller. At approximately 1,500 vph on the main street, the four-lane option becomes "better" (i.e. delay differences are positive) indicating that the delay with the three-lane option is greater than that of the four-lane. For the higher percentages of left-turns, delay differences remained practically the same as the volumes on the main street increased and for all combinations the four-lane option was "better" than the three-lane alternative. These data indicate that there is a region for low volumes on the main and side streets that the road diet option will result in an operational advantage, i.e. lower delays, than the four-lane option.

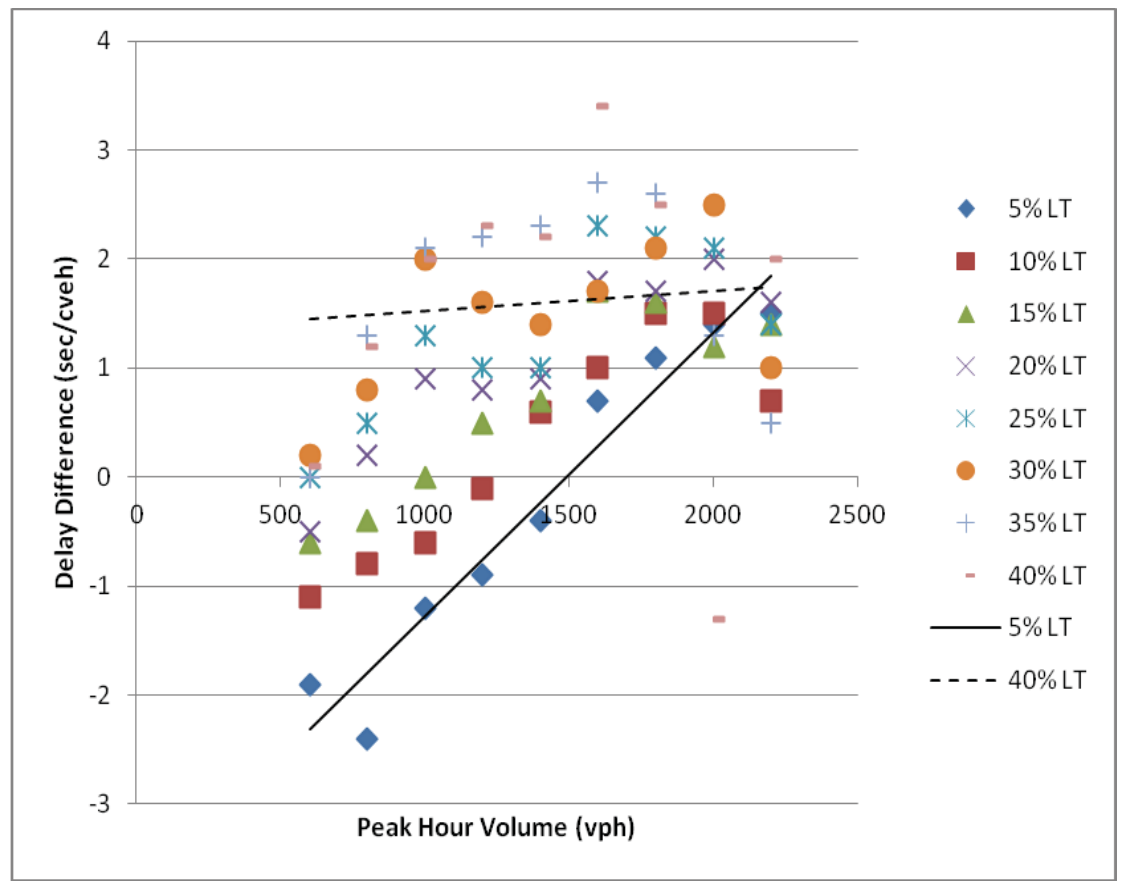

Figure 1 Signalized intersection delay differences (3-4), 300 vph side street volume

The cross street volume of 700 vph also showed similar trends for the low left-turn percentages (Figure 2). The data shows that at low volumes the three-lane option is "better" than the fourlane (i.e. negative delay differences) and as the volumes increase the delay differences become smaller. At approximately 1,750 vph on the main street, the four-lane option becomes "better" 
(i.e. delay differences are positive) indicating that the delay with the three-lane option is greater than that of the four-lane. For the higher percentages of left-turns, delay differences showed a reducing trend as the volumes in the main street increased and for all combinations the fourlane option was "better" than the three-lane alternative. The data indicates the advantage of the three-lane option for certain combinations of left-turn percentages and main street volume for this side street volume.

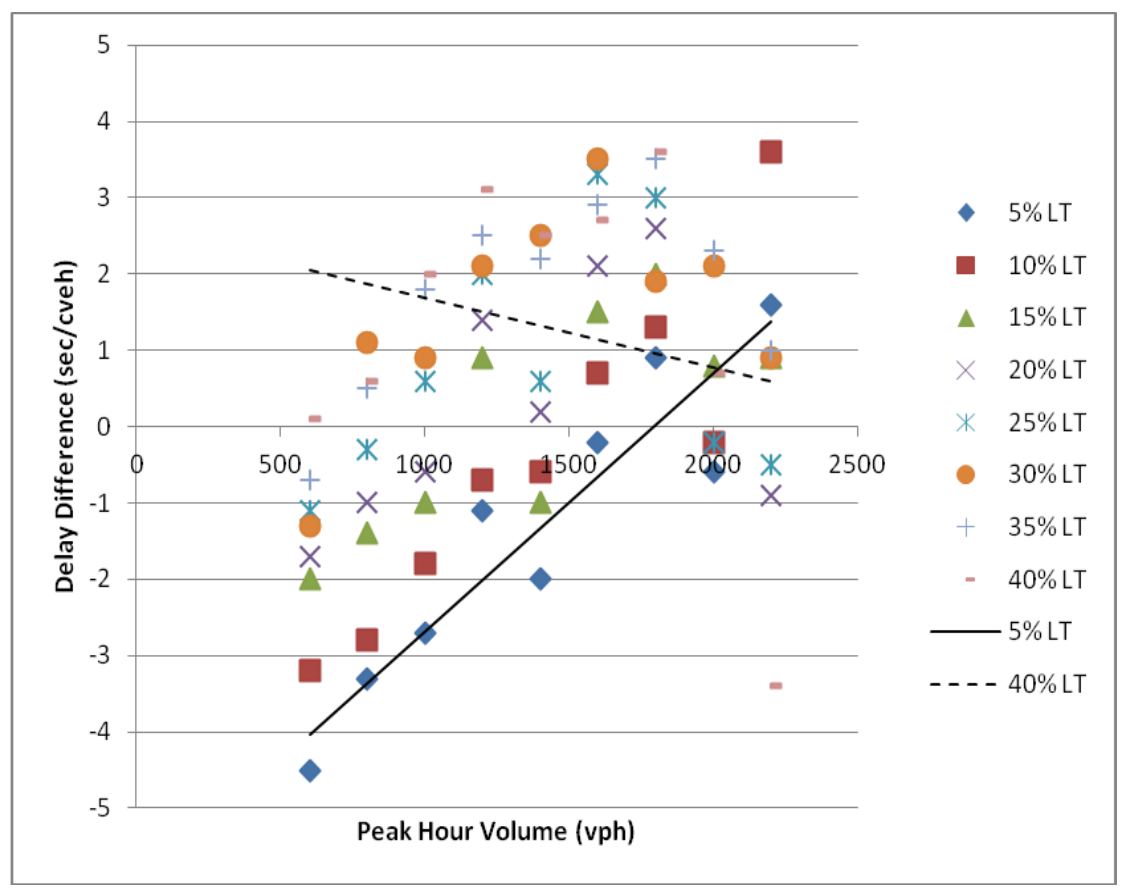

Figure 2 Signalized intersection delay differences (3-4), 700 vph side street volume

The effect of high side street volumes was evaluated to determine whether the noted trends hold (Figure 3). The data shows similar trends for both low and high left-turn percentages as those observed in the other side street volumes. It should be noted that this analysis only includes volumes up to 1,800 vph along the major street as the high side street volumes create lower capacity on the main street thus resulting in oversaturated conditions.

The data in Figures 1, 2, and 3 indicates that there are certain conditions where the road diet could be the preferred option over a four-lane alternative. The data also indicates that there is an effect of the side street volume in determining these conditions and that the combination of main and side street volumes needs to be considered in determining whether the road diet is appropriate. Finally, the left-turn percentage has an effect and this should be considered in conjunction with the traffic volumes.

Of interest is also the magnitude of the differences in delays observed in this analysis. Most delay differences were small (less than 5 seconds for the low side street volumes), indicating practically no significant operational gains or losses between the two options (Table 2). The average values for each side street volume for all left-turn percentages evaluated were less than $1.0 \mathrm{sec} / \mathrm{veh}$ indicating again that the delay differences are not significant. 


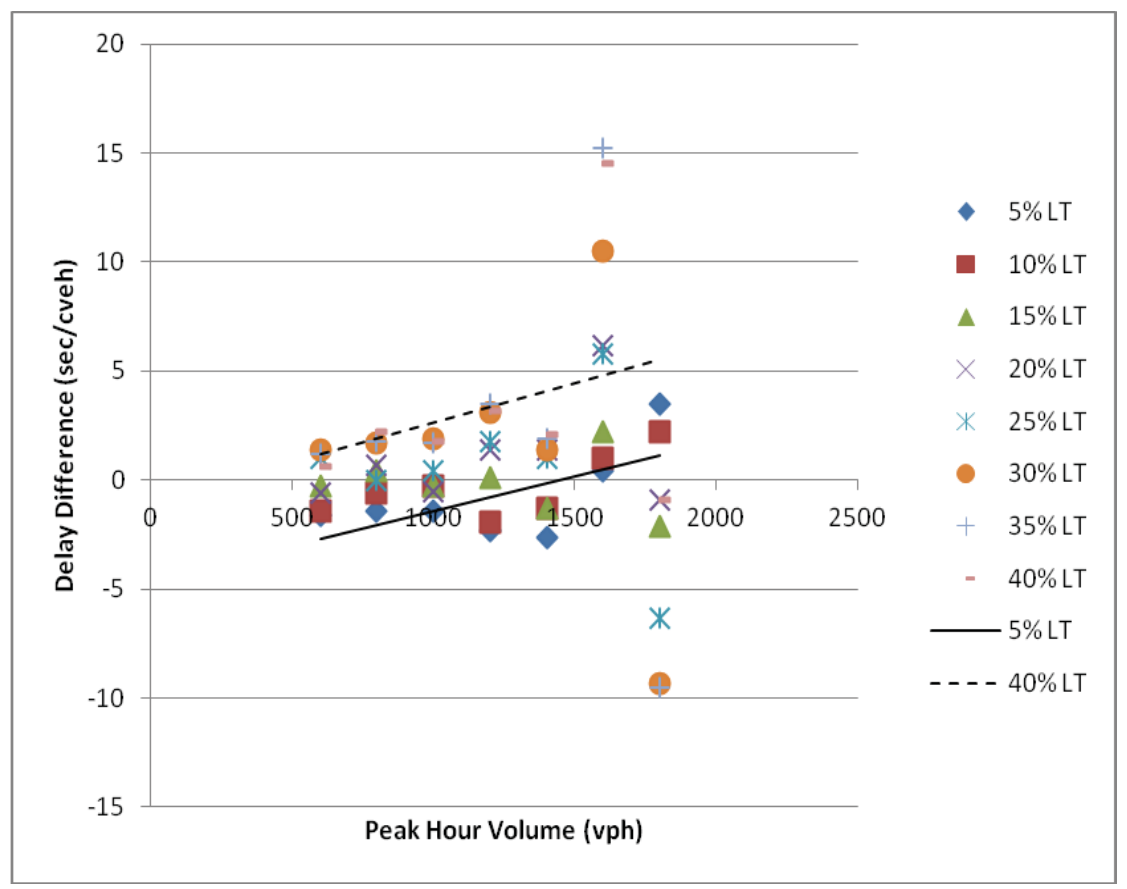

Figure 3 Signalized intersection delay differences (3-4), 1,300 vph side street volume

Table 2 Range of delay differences by side street volume

\begin{tabular}{cccc}
\hline $\begin{array}{c}\text { Side Street } \\
(\mathrm{vph})\end{array}$ & $\begin{array}{c}\text { Min } \\
(\mathrm{sec})\end{array}$ & $\begin{array}{c}\text { Max } \\
(\mathrm{sec})\end{array}$ & $\begin{array}{c}\text { Avg } \\
(\mathrm{sec})\end{array}$ \\
\hline 300 & -2.4 & 3.4 & 0.98 \\
700 & -4.5 & 3.6 & 0.50 \\
1300 & -9.5 & 15.5 & 0.94 \\
\hline
\end{tabular}

Another aspect that could be of interest regarding the operational efficiency of road diets is the resulting queue lengths. A queuing evaluation was completed using the same simulation scenarios presented above. The analysis showed that overall the road diet produced similar or smaller queue lengths than the four-lane roads. The effect of the side street volume was considered here as well and the results for the same three volumes used in the delay analysis are shown. It should be noted again that a negative number indicates that the three-lane road will produce shorter average queues than the four-lane option. The simulation output provides an estimate of the average queue by lane. The average queue difference here was taken as difference between the maximum average queue lengths for the three-lane, i.e. considering the queue lengths of the through and left-turn lane, to the maximum average queue length for the four-lane, where both lanes are evaluated.

For the 300 vph side street volume and low left-turn percentages scenario, the four-lane option will result in shorter average queues than the three-lane option (Figure 4). For the high left-turn percentages, the three-lane is a better option than the four-lane demonstrating lower queue lengths, and these gains increase (as the slope of the line indicates) with increasing main street volumes. 


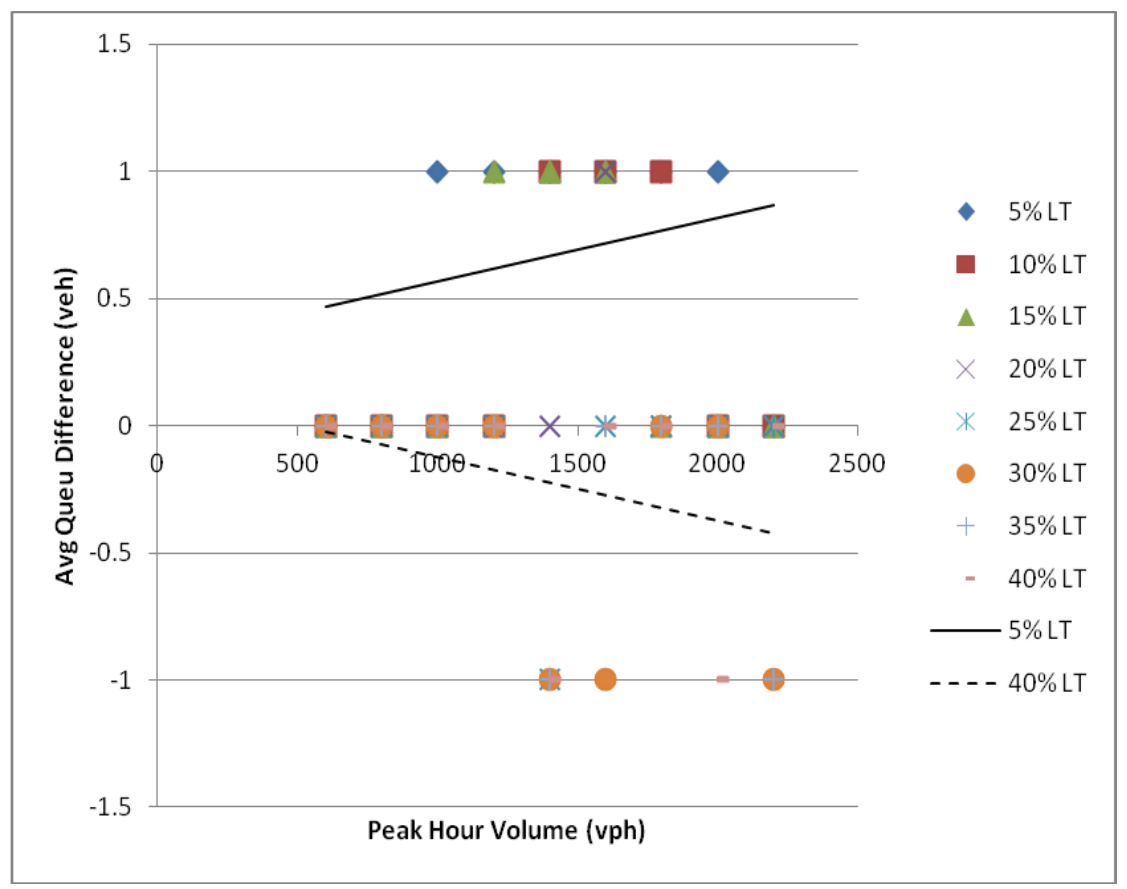

Figure 4 Signalized intersection average queue differences (3-4), 300 vph side street volume

The evaluation of the 700 vph side street volume showed a similar trend (Figure 5). Low left-turn percentages showed that the road diet is the better option for low main street volumes. At about $750 \mathrm{vph}$ the four-lane option shows shorter queues. High left-turn percentages showed that the road diet is the better option for all main street volumes and the gains increased with increasing main street volumes.

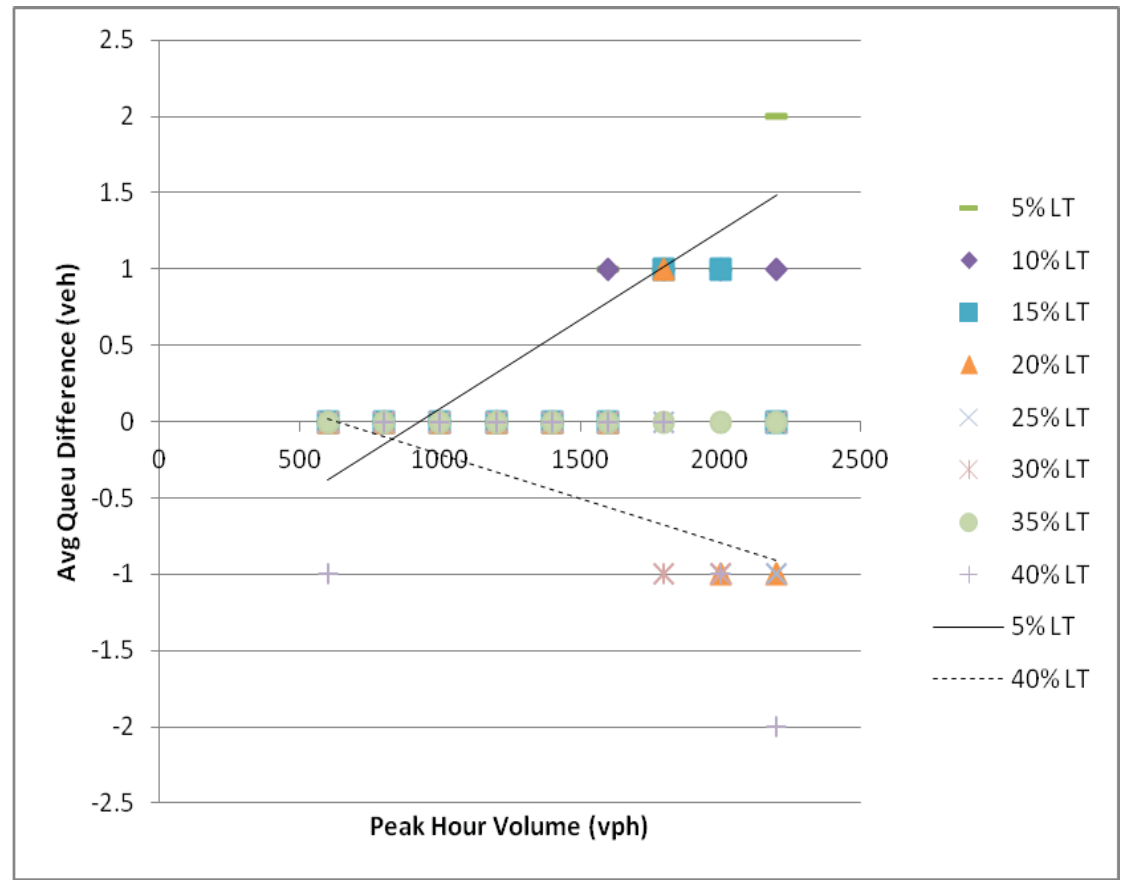

Figure 5 Signalized intersection average queue differences (3-4), 700 vph side street volume 
The consideration of the same data for the 1,300 vph side street volume produced similar trends but with larger differences between the two options (Figure 6). The data here again shows that the road diet will be a "better" option than the four-lane alternative for the high left-turn percentages, but the differences in queues were smaller than those noted in the other side street volume scenarios.

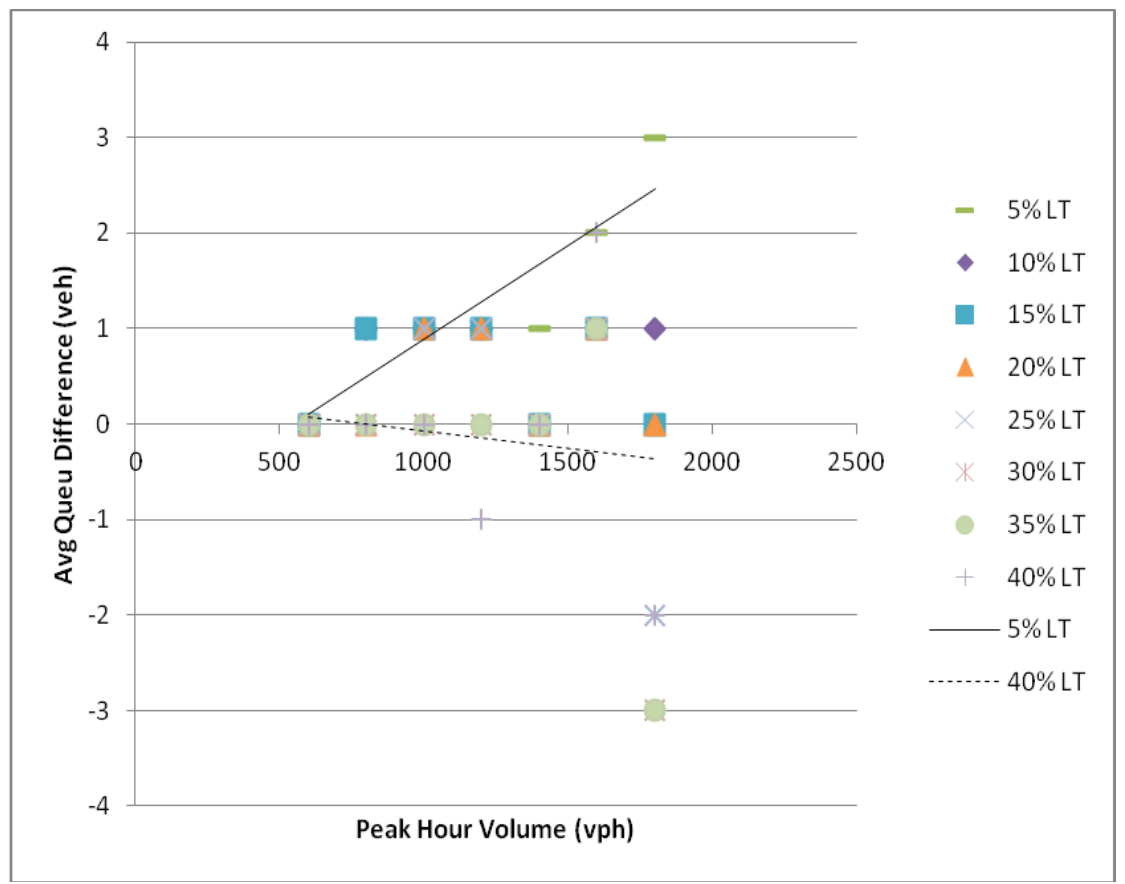

Figure 6 Signalized intersection average queue differences (3-4), 1,300 vph side street volume

A review of the range of values noted here also indicates that overall there are no significant differences between the two options when the average queue lengths are considered (Table 3). The largest differences were noted for the 1,300 vph side street volume scenario, and the averages for each side street volume were less than 0.5 vehicles, again virtually the same.

Table 3 Range of average queue differences by side street volume

\begin{tabular}{cccc}
\hline $\begin{array}{c}\text { Side Street } \\
\text { (vph) }\end{array}$ & $\begin{array}{c}\text { Min } \\
\text { (veh) }\end{array}$ & $\begin{array}{c}\text { Max } \\
\text { (veh) }\end{array}$ & $\begin{array}{c}\text { Avg } \\
\text { (veh) }\end{array}$ \\
\hline 300 & -1 & 1 & 0.07 \\
700 & -2 & 2 & 0.03 \\
1300 & -3 & 3 & 0.30 \\
\hline
\end{tabular}

\section{Unsignalized Intersections}

Delay and queuing analysis was also conducted for unsignalized intersections. In this case, all delays along the major road were very small (less than $4.5 \mathrm{sec} / \mathrm{veh}$ ) and the three-lane option had slightly higher delays than the four-lane option. However, these differences were very small and most were less than $2.5 \mathrm{sec} / \mathrm{veh}$, with the majority (56 percent of the cases) of the delay differences being $1 \mathrm{sec} / \mathrm{veh}$ or less. 
The significant differences in the unsignalized intersections were noted for the delays on the side street approaches. For all scenarios tested, the delays on the side street for a road diet conversion were smaller than those observed for the four-lane road. The data was analyzed in a similar manner as that of the signalized intersections to evaluate the effect of left-turn percentages in conjunction with the main street volumes. The delay difference noted here is computed in the same manner as above, i.e. delay of three-lane minus delay of four-lane option. In this case, also, negative differences indicate that the three-lane alternative results in lower delays than the four-lane alternative for the side street approach.

As shown in Figure 7, the road diet produces lower delays for the side street than the four-lane option for all left turn percentages with a total approach volume of $300 \mathrm{vph}$. The data also indicate that as the main street volumes increase, the differences in delays increase for all leftturn percentages. .

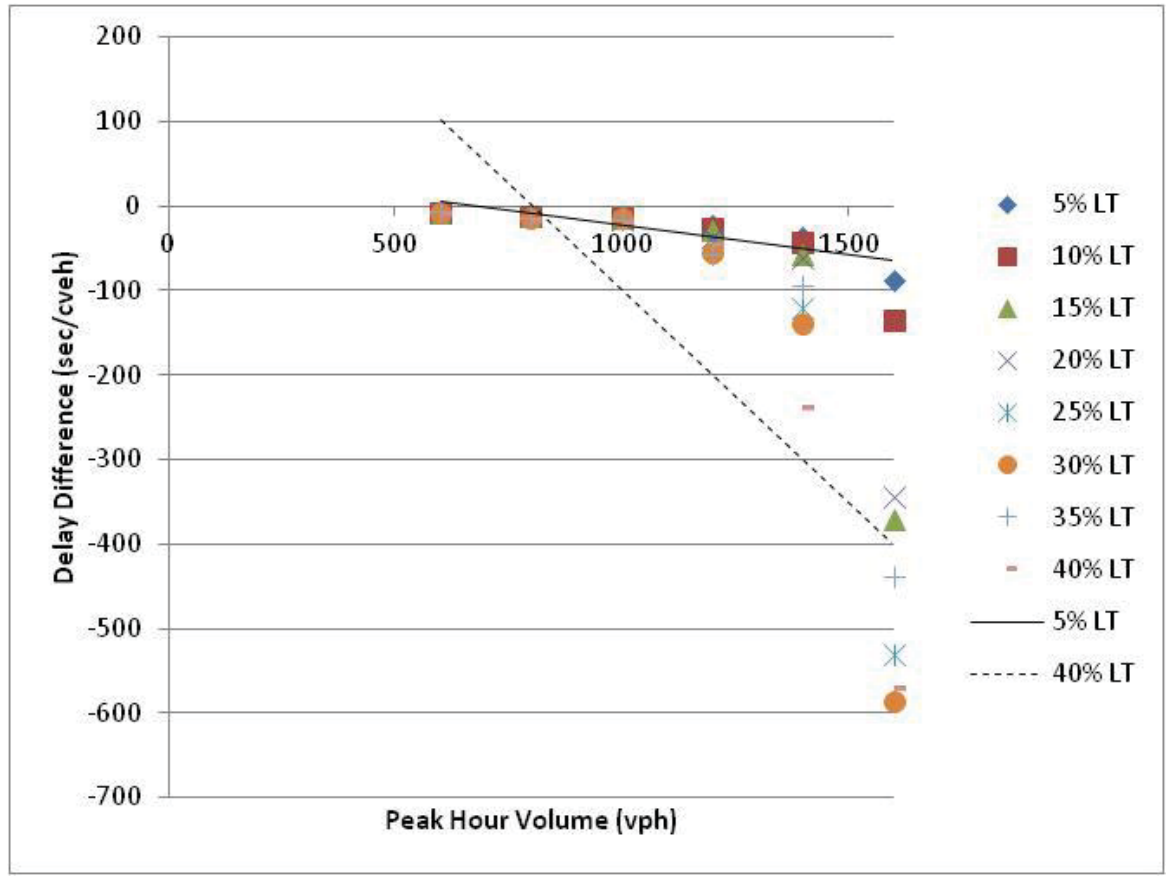

Figure 7 Unsignalized intersection side street delay differences (3-4), 300 vph side street volume

The same trend was noted for the lower side street volumes, where for all cases examined, the side street delays improved for any combination of main street volume and left-turn percentages and these differences increased as both volumes and left-turn percentages increased. It is apparent that significant gains could materialize from road diet conversions for unsignalized intersections; an aspect that may outweigh the minor delay increases at signalized intersections as was discussed above.

The average queue length for side street approaches was also examined to determine the potential effect of the road diet conversions. The data shows that in all cases the road diet will result in smaller queues (Figure 8). The data shows that as traffic volumes along the main street increase, the differences in the average queue length along the side street are diminishing. 
However, road diets maintain the advantage (i.e. shorter average queues on the side street) for almost all main street volumes examined. This indicates that benefits from road diets could materialize for most situations. It should be noted that for all side street volumes similar trends are observed, and only the trend of the $300 \mathrm{vph}$ side street volume is presented.

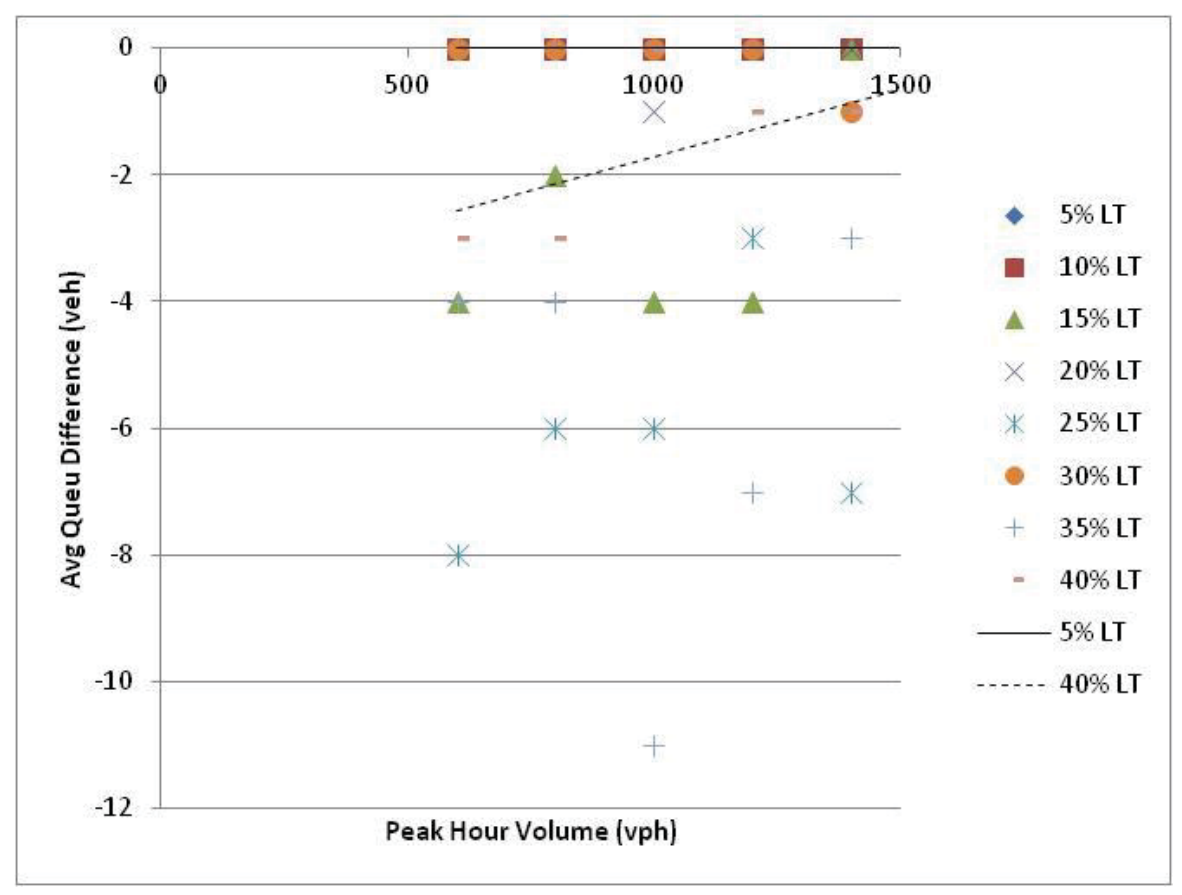

Figure 8 Unsignalized intersection average side street queue differences (3-4), 300 vph side street volume

\section{Operations Models}

The analysis completed here was also used to develop prediction models that could allow for establishing guidelines and identifying the conditions under which road diets could improve the operational efficiency of the roadway. The Statistical Package for Social Sciences (SPSS) was utilized to develop these models. Linear regression models were used to model the delay for each condition, i.e. three- and four-lane options, and identify the variables that could predict these differences. The variables considered include the volumes of the main and side streets as well as left-turn percentage.

The model for the three-lane included all three variables of concern and had a good predictive power with an $\mathrm{R}^{2}$ of 0.48 . The model coefficients are summarized in Table 4 . The four-lane model also utilized all three variables but the predictive power was lower $\left(R^{2}=0.28\right)$. The variables included in the equations reflect the effect of traffic volumes on delays and their signs indicate that increasing volumes will result in greater delays.

Table 4 Coefficients for predictive models 


\begin{tabular}{lcccc}
\hline \multirow{2}{*}{ Variable } & \multicolumn{2}{c}{ Three-lane } & \multicolumn{2}{c}{ Four-lane } \\
\cline { 2 - 5 } & Parameter & P value & Parameter & P value \\
\hline Intercept & -29.113 & 0.00 & -47.968 & 0.00 \\
Main street volume (vph) & 0.013 & 0.00 & 0.022 & 0.00 \\
Side street volume (vph) & 0.025 & $0 / 00$ & 0.037 & 0.00 \\
Left-turn percentage (number) & 0.313 & $0 / 00$ & 0.314 & 0.00 \\
\hline
\end{tabular}

These models can be used to define the scenarios where the three- and four-lane options produce a delay difference of zero, i.e. both options will perform equally well. This will produce the line of equality which can then be used to establish the regions where road diets are advisable and those where they are not. Figure 9 presents this concept where to the right of the line, road diets are not recommended, while to the left of the line road diets are considered appropriate.

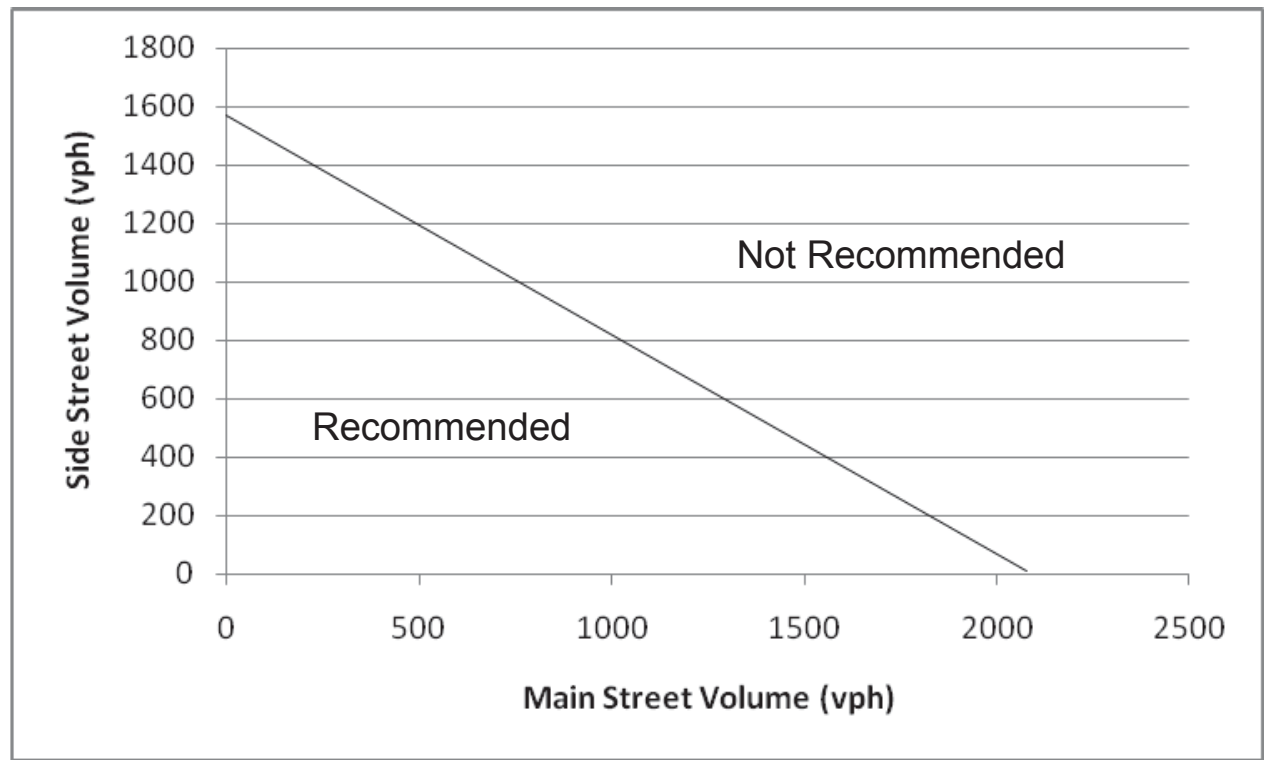

Figure 9 Guideline for operational performance at signalized intersections

The guideline developed is based only on main and side street volumes, since it was determined that the operation of the signalized intersection is the critical aspect for a road diet conversion. While left turn percentage was shown to be a significant parameter for the capacity and delay of an intersection, it has the same effect for both the four-lane and three-lane sections and therefore provides no differentiation between the alternatives. In other words, once the effect of the main and side street volumes on delays have been accounted for, the left-turn percentage does not contribute any additional delay to the difference between the two options.

As unsignalized access points do not interrupt traffic flow on the primary street, they are not a significant determinant of the success or failure or a road diet project. Even unsignalized access points with high volumes of left turn traffic on the major street will continue to operate at acceptable levels of service while signalized intersections may fail due to the reduced mainline capacity. The primary concern for unsignalized access points is overlapping left turn movements within the two-way left turn lane, which is avoided with the four-lane section. The existing KYTC 
auxiliary turn lane policy currently addresses this concern by recommending against the use of TWLTLs for access point densities greater than 85 access points per mile (KYTC 2009).

\section{Signalized Intersection Safety}

Safety analysis of road diet performance was also conducted using the same simulated scenarios through application of SSAM software. It should be noted that this analysis uses a surrogate measure for safety, i.e. number of conflicts in the traffic stream, and does not directly predict the number of crashes, though research has shown a direct correlation between the two measures (Gettman et al 2008). The results presented here also use the same relationship between the two options as was done with the operational analysis, i.e. negative differences in conflict numbers indicate that the three-lane option will perform better than the four-lane option, while positive numbers indicate better performance by the four-lane cross-section. This analysis only considered main street conflicts, since these are the safety impacts of major concern.

The use of the simulation models in developing a safety analysis in lieu of using before-andafter data was considered more appropriate for several reasons. First, the models allow for an evaluation of a greater range of volumes than the available case studies. Second, there are not many road diet implementations in Kentucky that would provide for a robust analysis and allow for drawing any conclusions regarding the safety implications of road diet installations. Third, the road diet implementations have a variety of other factors (such as varied number of access points, land use, turning percentages, and cross sectional elements) that could create greater uncertainty than the models with their controlled environment. Finally, safety consequences from the road diet implementation could not be easily separated from other issues stemming from prior problems (such as access control and improper transition to and from the road diet section) on the site.

Safety analysis was conducted for rear end and sideswipe conflicts. It did not include the analysis of angle crashes as the simulation methodology used is not capable of accurately reflecting angle crash exposure. The primary reason for this is that angle crashes often result from poor sight distance, which is not able to be modeled effectively within the simulation programs. A review of the case studies discussed in Appendix A indicates that road diets are effective in reducing left turn angle crashes resulting from offset left turn lanes. However, an increase in angle crashes was observed at an access point that was placed near the transition zone between the three- and four-lane sections. Therefore, road diets may be effective in addressing angle crash problems, but careful review of the individual site should be performed.

\section{Rear End Conflicts}

The simulation results analyzed indicated that road diets may have mixed results in terms of rear end conflicts. In general, rear end conflicts may increase as the result of road diets on roadways with a low volume of left turning vehicles (Figure 10). This increase is due to the decreased capacity of the through movement, brought about by the removal of one through lane. This finding is consistent with other studies which show an increase in rear end crashes with increasing volumes (Geedipaly et al 2010). 


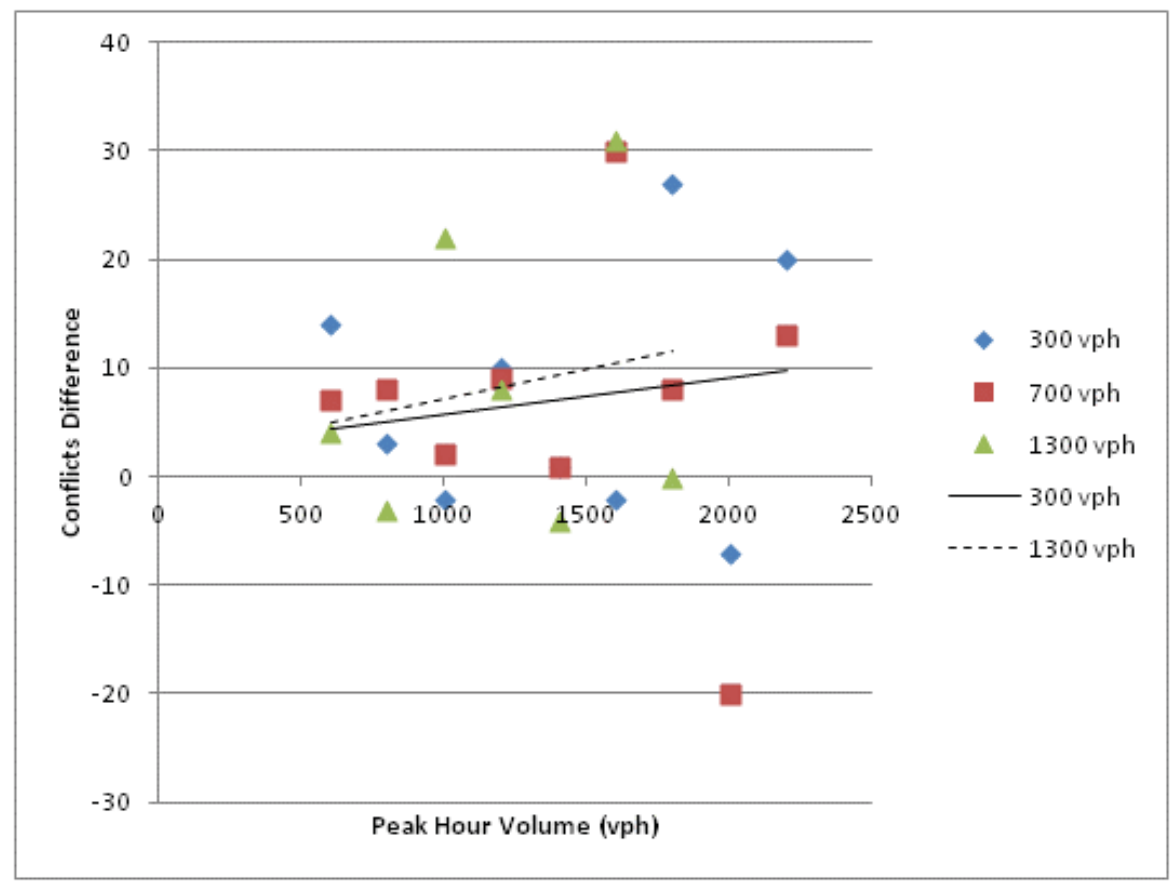

Figure 10 Difference in main street rear end conflicts between four- and three-lane signalized intersections (3-4) by side street volume, 5 percent left turns

For moderate left turn percentages (15-25 percent), road diets generally showed a reduction in rear end conflicts compared to the four-lane option (Figure 11). This reduction can be attributed to the removal of speed differential between left turn vehicles and through vehicles which offsets the potential for rear end conflicts in the through lane. For this range of volumes, this may be considered an indication that road diets could reduce such conflicts even though both options could operationally be the same. For high side street volumes (e.g., 1,300 vph) the differences between the two options decrease demonstrating the effect of the side street volume (and increased congestion on the safety performance of the road). 


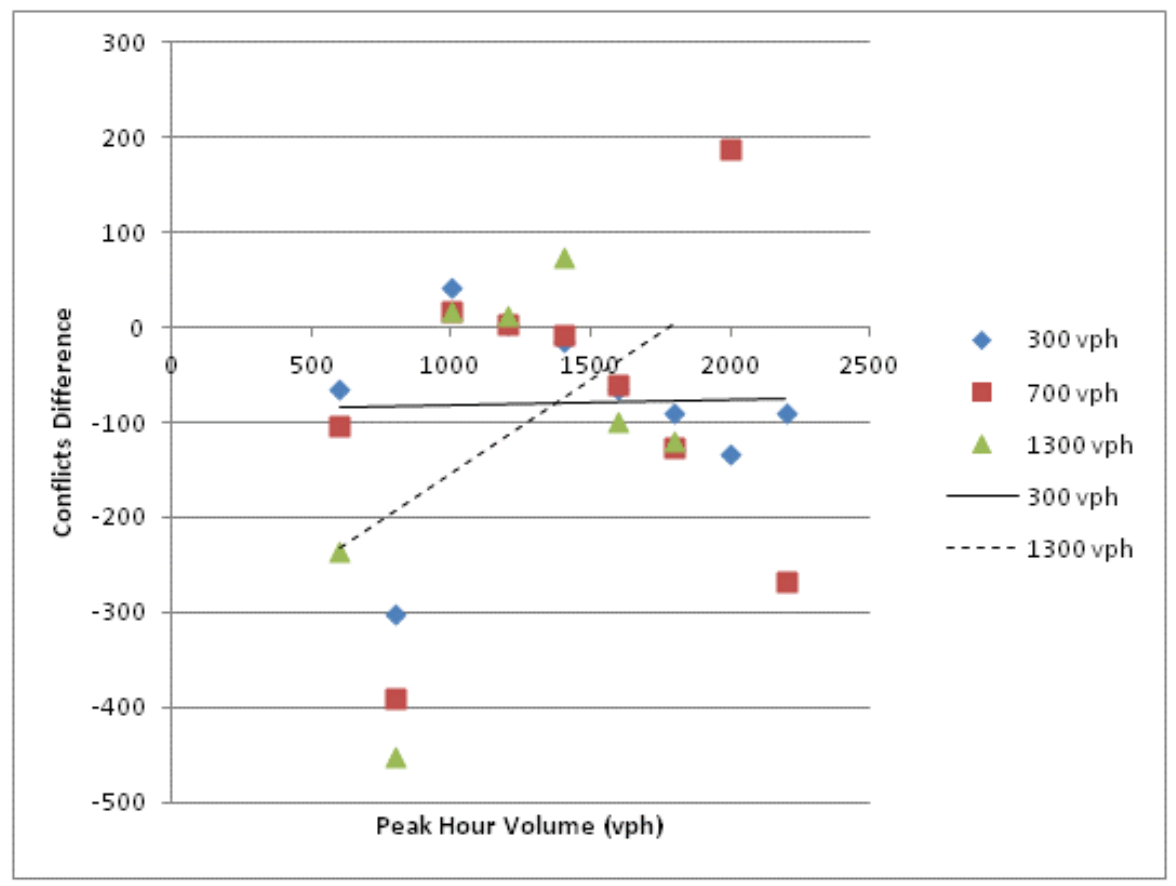

Figure 11 Difference in main street rear end conflicts between four- and three-lane signalized intersections (3-4) by side street volume, 20 perecnt left turns

Finally, for high percentages of left turns (e.g., 35 percent) the road diet will result in more rear end conflicts than the four-lane option (Figure 12). In this case for most volume scenarios the difference is positive, i.e. more conflicts with the road diet. The overall trend of increasing conflicts with increasing volumes is observed here as well. 


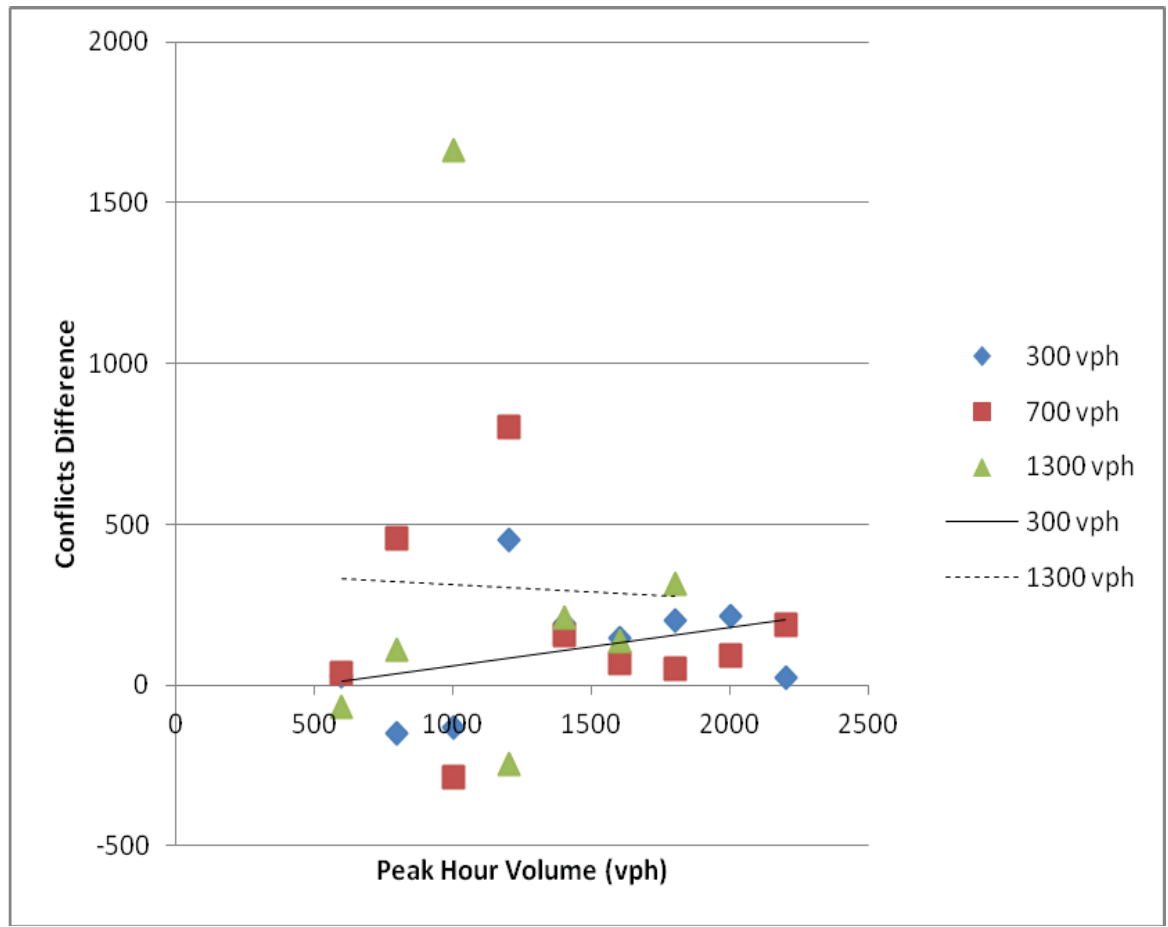

Figure 12 Difference in main street rear end conflicts between four- and three-lane signalized intersections (3-4) by side street volume, 35 percent left turns

It should be noted that the 40 percent left-turns produced high variations and were considered as outliers in the analysis and thus were excluded in the results discussed here.

In summary, the data indicates that there are scenarios when the road diet will result in lower rear end conflicts and these include mid-range volumes and mid-range left-turn percentages. At high volumes with high left-turn percentages the road diet may increase congestion and thus rear end conflicts. Roadways with low volumes and low left turn percentages do not typically have rear end problems and thus the reduction in capacity may cause a slight uptick in rear end conflicts.

\section{Lane Change Conflicts}

The simulation results analyzed showed that the number of lane change conflicts for the main street will decrease with a road diet at the signalized intersections. This was an expected result, since the number of lanes is reduced and thus the opportunity for such conflicts is reduced or even eliminated. The difference in the number of conflicts is affected again by the left-turn percentages where the difference in the number of conflicts increases (greater negative numbers indicate a larger benefit for the road diet scenario) as the volume increases (Figure 13). This could be attributed to the lack of the extra lane in the road diet which results in fewer opportunities for lane change conflicts. The data also shows that, in general, increasing left-turn percentages resulted in larger differences between the two options, in favor of the road diets. There was no discernible effect of the side street volume for these conflicts and all showed similar trends as those presented in Figure 13. 


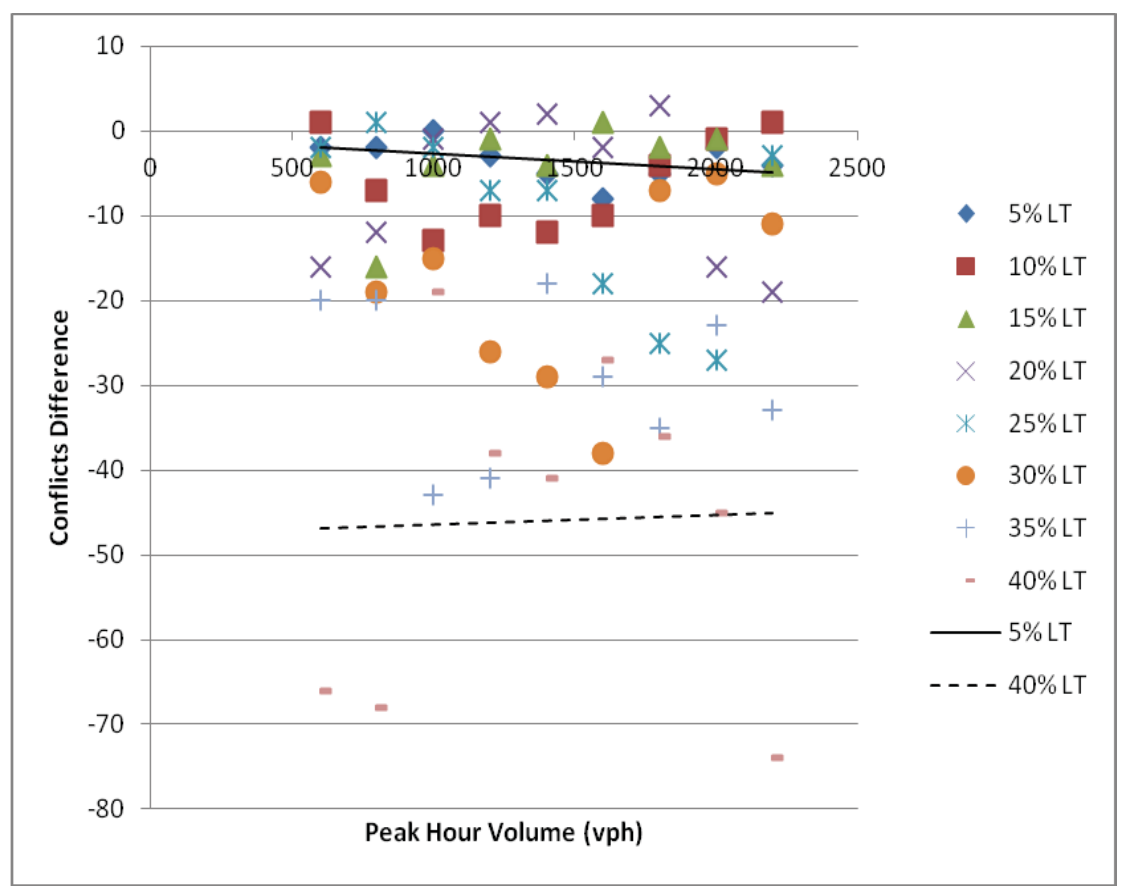

Figure 13 Difference in main street lane change conflicts between four- and three-lane signalized intersections (3-4), 700 vph side street volume

\section{Unsignalized Intersection Safety}

A similar evaluation of main street safety performance was completed for unsignalized intersections to evaluate the road diet effect. Similarly, the difference in the number of conflicts along the main street is considered as the metric here and a negative difference indicates a "safer" performance for the road diets.

\section{Rear End Conflicts}

The simulation analysis showed that for unsignalized intersections rear end conflicts will increase for most combinations with a road diet installation. For low left-turn percentages, a small increase is noted in rear end conflicts with increasing main street volume (Figure 14). This trend reversed for higher left-turn percentages where the differences decrease with increasing main street volumes. This could be attributed to the presence of the two-way left-turn lane which could have a greater effect in higher volumes and left-turn percentages. 


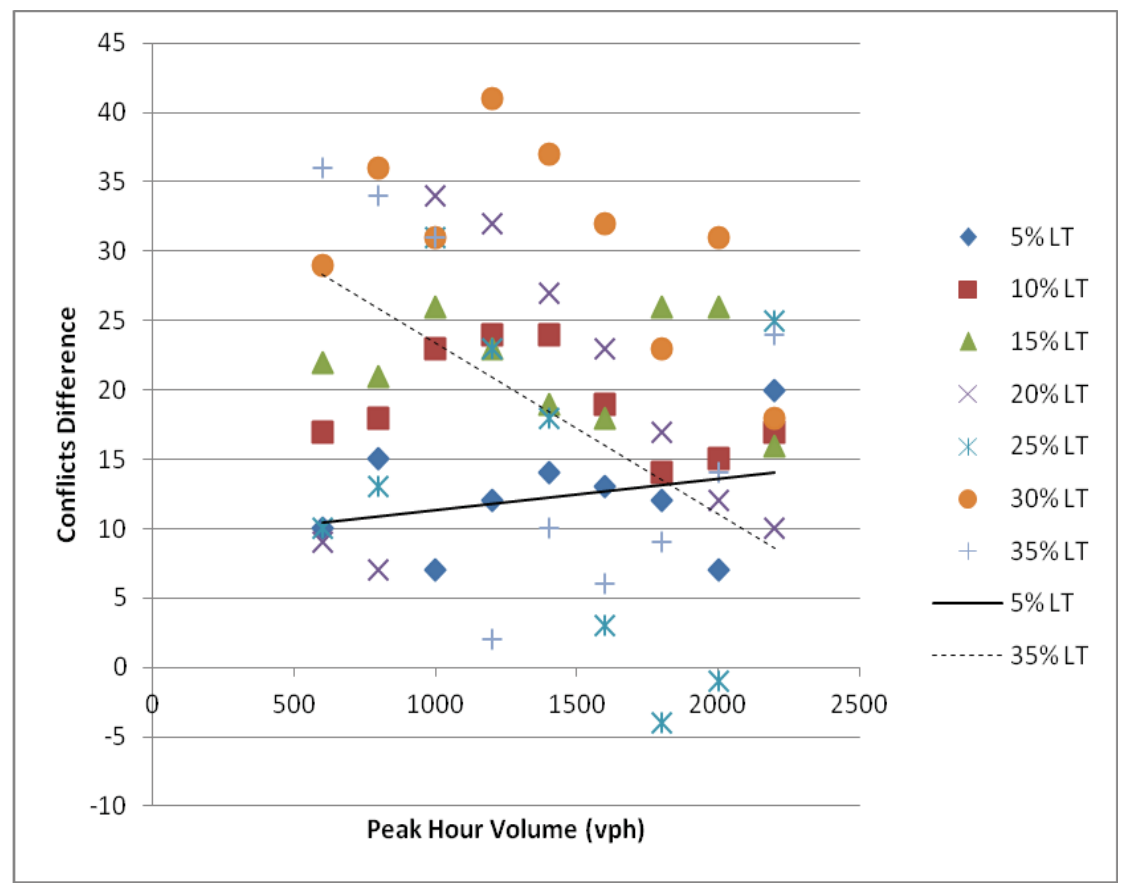

Figure 14 Difference in main street rear end conflicts between four- and three-lane unsignalized intersections (3-4), 700 vph side street volume

\section{Lane Change Conflicts}

The simulation results analyzed showed overall that the number of lane change conflicts along the main street will increase with a road diet at the unsignalized intersections (Figure 15). However, there were combinations of main street volumes and left-turn percentages for which the three-lane option resulted in fewer conflicts than the four-lane option. The data shows that greater left-turn percentages resulted in larger differences between the two options in favor of the road diet option. 


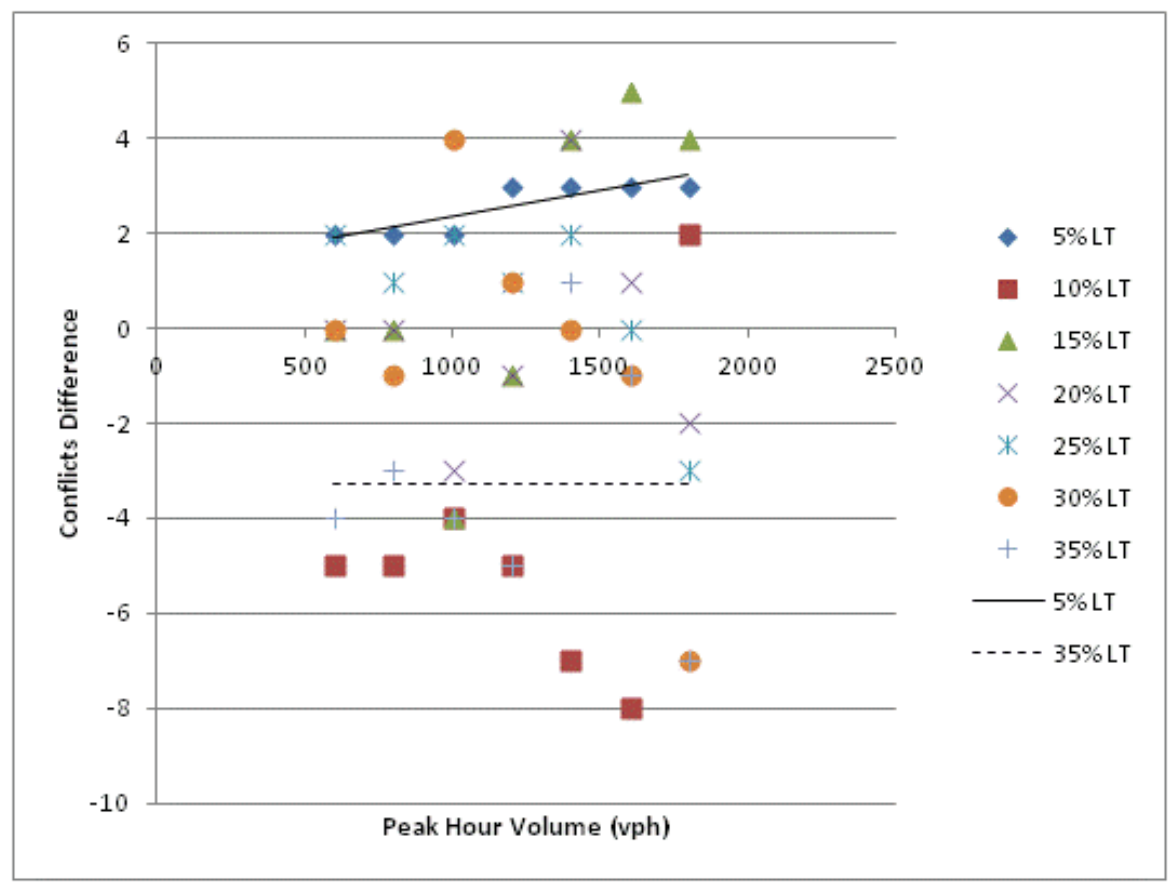

Figure 15 Difference in main street lane change conflicts between four- and three-lane unsignalized intersections (3-4), 700 vph side street volume

\section{Safety Models}

The analysis completed here was also used to develop conflict prediction models for both the three-lane and four-lane conditions to assist in identifying the conditions under which road diets could improve the roadway safety. The Statistical Package for Social Sciences (SPSS) was utilized to develop these models. Linear regression models were used to model the conflicts and identify the significant variables. The variables considered include the main street volume, side street volume and left-turn percentage.

Model variables and $\mathrm{R}^{2}$ values are provided for the three-lane and four-lane models for both rear end and lane change conflicts (Table 9). All models developed included all variables, except for the three-lane rear end conflicts, which did not include the left turn percentage as a significant parameter. The positive signs of the coefficients for all variables in all models indicate that increasing volumes will result in a greater number of conflicts. All models had $\mathrm{R}^{2}$ values greater than 0.70 indicating the included variables explain over 70 percent of the variability in the data set. 
Table 5 Coefficients for predictive models

\begin{tabular}{|c|c|c|c|c|c|}
\hline \multirow[b]{2}{*}{ Conflict } & \multirow[b]{2}{*}{ Variable } & \multicolumn{2}{|c|}{ Three-lane } & \multicolumn{2}{|c|}{ Four-lane } \\
\hline & & Parameter & $P$ value & Parameter & $\mathrm{P}$ value \\
\hline \multirow{3}{*}{ Rear end } & Constant & 88.322 & 0.00 & 81.037 & 0.00 \\
\hline & $\mathrm{v} / \mathrm{c}$ ration exponent & 2.0433 & 0.00 & 1.8927 & 0.00 \\
\hline & $\mathrm{R}^{2}$ & \multicolumn{2}{|c|}{0.93} & \multicolumn{2}{|c|}{0.87} \\
\hline \multirow{5}{*}{$\begin{array}{l}\text { Lane } \\
\text { change }\end{array}$} & Intercept & -8.777 & 0.00 & -46.072 & 0.00 \\
\hline & Main street volume (vph) & 0.005 & 0.00 & 0.027 & 0.00 \\
\hline & Side street volume (vph) & 0.003 & 0.00 & 0.025 & 0.00 \\
\hline & Left-turn percentage (percent) & 0.271 & 0.00 & 0.440 & 0.00 \\
\hline & $\mathrm{R}^{2}$ & \multicolumn{2}{|c|}{0.72} & \multicolumn{2}{|c|}{0.76} \\
\hline
\end{tabular}

These models can be used to define the scenarios where the three- and four-lane options produce a conflict difference of zero, i.e. both options will perform equally well. This will produce the line of equality which can then be used to establish the regions where road diets are advisable and those where they are not.

In addition to major and minor street volumes, left-turn percentage was shown to be a significant factor in predicting rear end crashes. As noted above, there are scenarios where the four-lane section will produce fewer rear end conflicts than the road diet. This is primarily due to the increased congestion resulting from the reduction in the number of through lanes. However, as the left-turn percentage increases the road diet is shown to reduce rear end conflicts. Figure 16 shows the line of equality for five percent, 20 percent and 35 percent left-turns as a function of the main and side street volumes. In the figure, the main and side street volume combinations below the line indicate that rear end conflicts would be anticipated to be reduced with a threelane section, compared to a four-lane section.

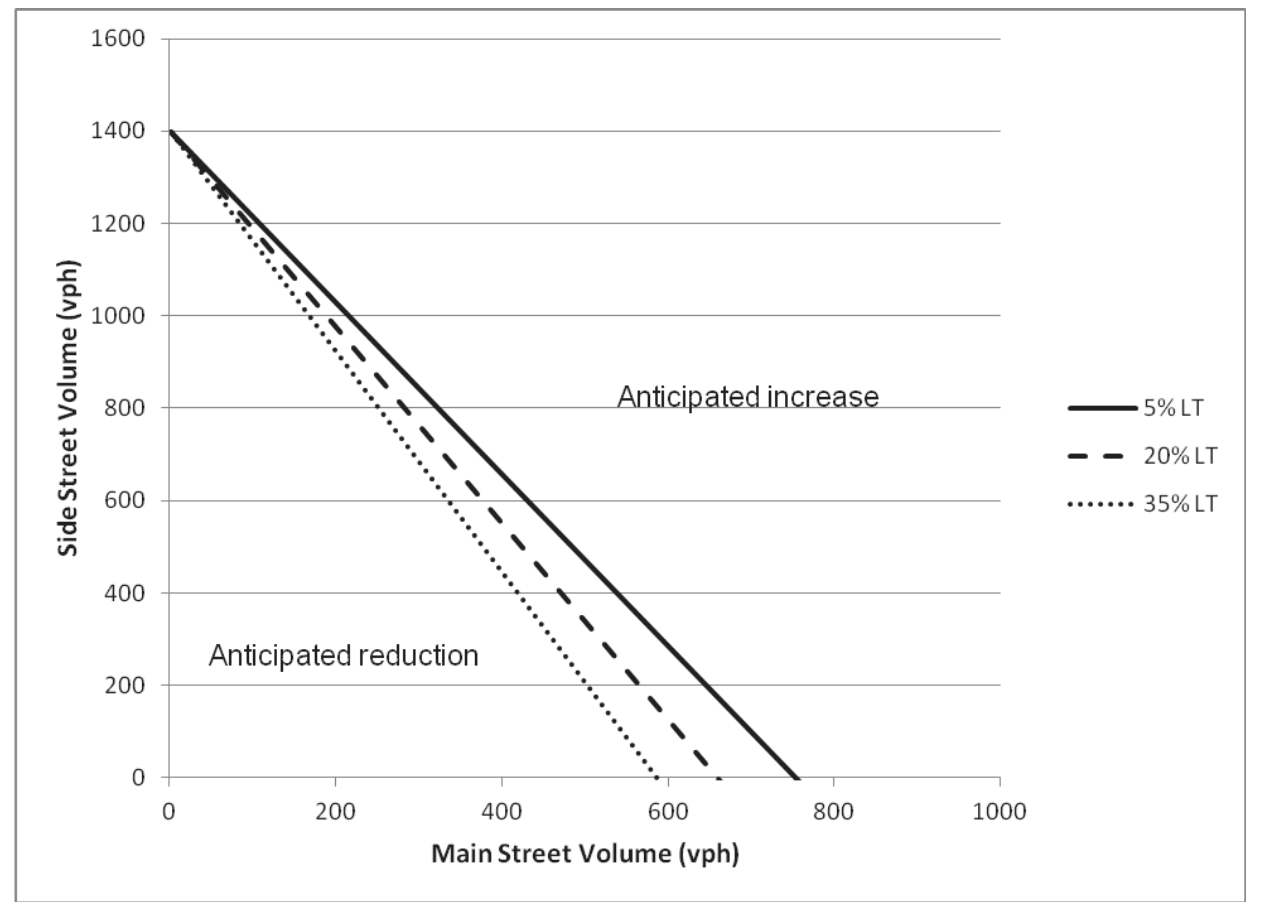

Figure 16 Guidelines for rear end conflicts by main and side street volume and percent left-turns 
A similar model was developed for lane change conflicts. The results of this analysis are shown in Figure 17. Left-turn percentage does not have as significant of an effect on lane changes as was evident for rear end conflicts. As shown in the figure, to the right of the line three-lane sections are anticipated to experience lower lane change conflicts, while to the left of the line, road diets are expected to have higher lane change conflicts than the four-lane section. Review of the lane change models, indicate that both the three-lane and four-lane sections have low lane change volumes in the lower volume areas below the equality lines; however, the four-lane section shows a significant increase in lane change conflicts at high volumes. Therefore, the potential for lane change conflicts is minimal in the lower volume range.

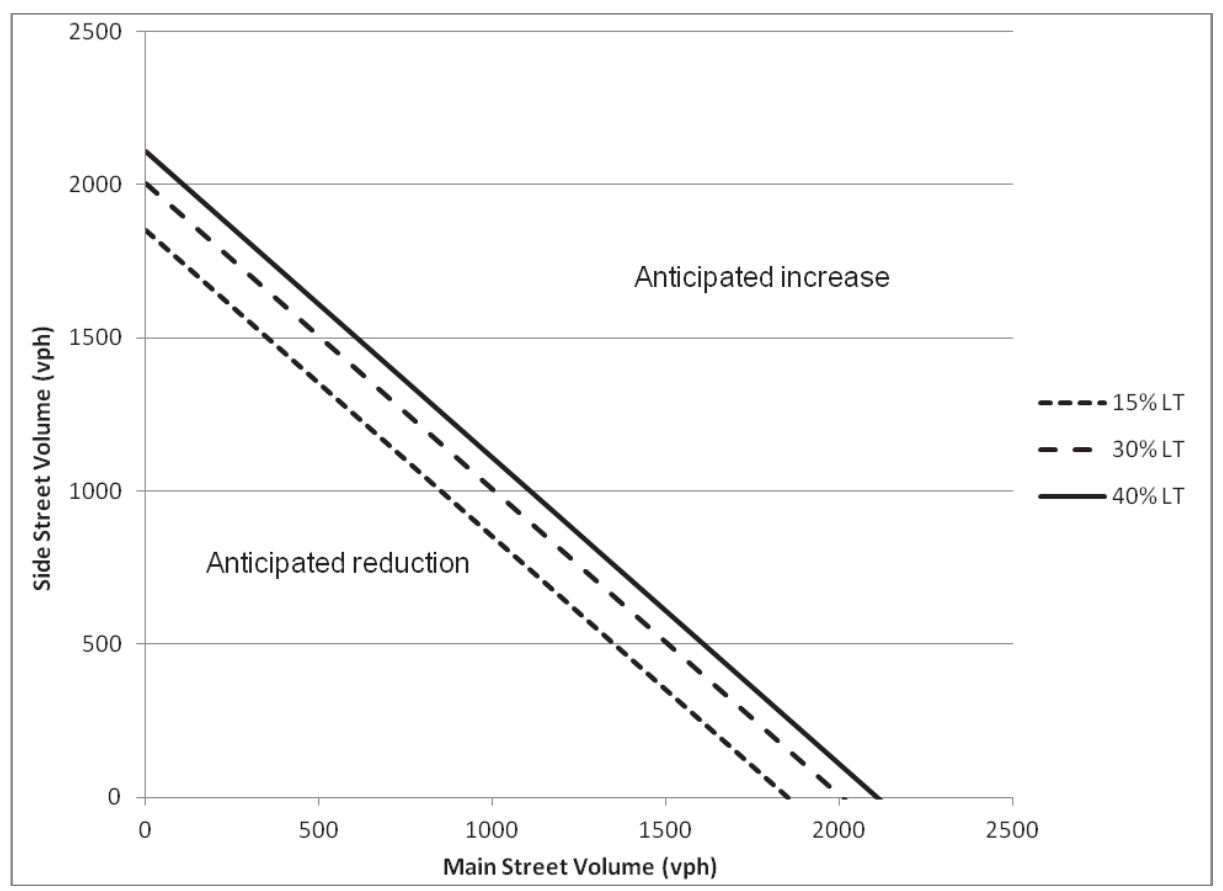

Figure 17 Guidelines for lane change conflicts by major/minor street volumes and percent leftturns

\section{Summary}

The simulation results from CORSIM support the assumption that a four-lane option will have a critical lane volume lower than that of a three-lane alternative. This could indicate an operational advantage of the four-lane option when compared to that of the three-lane. For signalized intersections, there are certain conditions where the road diet could be the preferred option over a four-lane alternative. The data also indicates that there is an effect of the side street volume in determining these conditions and that the combination of main and side street volumes needs to be considered in determining whether the road diet is appropriate. Another aspect that could be of interest regarding the operational efficiency of road diets is the resulting queue lengths. An evaluation was completed for this aspect using the same data. The analysis showed that overall the road diet produced smaller or the same average queue lengths than the four-lane roads.

A similar analysis for unsignalized intersections showed that all delays along the major road were very small and the three-lane option had slightly higher delays than the four-lane option. However, these differences were very small and most were less than $2.5 \mathrm{sec} / \mathrm{veh}$ with the 
majority (56 percent of the cases) of the delay differences being 1 sec/veh or less. The significant differences in the unsignalized intersections were noted for the delays along the side street. For all scenarios tested, the delays on the side street for a road diet conversion were smaller than those observed for the four-lane road, as a result of two-stage turning maneuvers provided by the two-way left-turn lane.

A review of safety aspects of the road diets was also conducted using the simulated cases. It should be noted that the analysis presented uses a surrogate measure for safety, i.e. potential number of conflicts, and not crashes. The simulation results showed that, in general, road diets will result in lower rear end conflicts for mid-range volumes and mid-range left-turn percentages. At high volumes with high left-turn percentages the road diet may increase congestion and thus rear end conflicts. Roadways with low volumes and low left-turn percentages do not typically have rear end problems and thus the reduction in capacity may cause a slight uptick in rear end conflicts. This was an expected result, since the left-turn vehicles can block the through lane increasing the probability of rear end conflicts.

The analysis for the lane change conflicts along the main street showed that at low volumes neither the three-lane or four-lane alternative had a high number of conflicts and the road diet produced less lane change conflicts at higher volumes. This was an expected result, since the number of lanes is reduced and thus the opportunity for such conflicts is also reduced. The difference in the number of conflicts is less affected by the left-turn percentages as was the rear end conflicts where the difference in the number of conflicts increases as the volume increases. This could be attributed to the lack of the extra lane in the road diet which results in fewer opportunities for lane change conflicts.

The analysis for the unsignalized intersections showed mixed trends for rear end conflicts indicating that there are combinations where the number of rear end conflicts will increase with a road diet while for others they decrease. This trend was dependent upon the cross street volume. For low cross street volumes, an increase is noted in read end conflicts with increasing main street volume. This trend was true for both low and high left-turn percentages where the differences increase with increasing left-turn percentages. The analysis of the lane changes for unsignalized intersections showed overall that the number of lane change conflicts along the main street will decrease with a road diet at the unsignalized intersections. For unsignalized intersections though, there were combinations of main street volumes and left-turn percentages for which the four-lane option resulted in fewer conflicts than the three-lane option. 


\section{CASE STUDIES}

Five road diet conversions in Kentucky were identified and evaluated to determine their overall effects and identify elements that could also assist in the development of the guidelines. A summary of each case study is presented here and an extended discussion of each case study is presented in Appendix A.

\section{US 27 Campbell County}

The road diet was completed in 2008 and covers 1.4 miles of the highway. The main cross section has two 11-foot lanes with a 12-foot two-way left-turn lane and 5-foot bicycle lanes in both directions. The before-after crash analysis conducted showed a 68 percent overall decrease in crashes with an accompanying decrease of 53 percent in rear end and 80 percent in sideswipe crashes. Between 2004 and 2010, there was a reduction in the ADT (from 10,600 vpd to 7,410 vpd) along this roadway section, though it should be noted that vehicles miles of travel statewide have stagnated or reduced during that time due to other economic factors. Examination of the crash rates indicated that the total crash rates as well as the total number of crashes was reduced, indicating that the crash reduction was due to the road diet installation. The travel time studies conducted did not demonstrate any significant congestion issues with an average travel speed of $21 \mathrm{mph}$ during the PM peak period. Overall, this road diet conversion could be considered as a successful installation.

\section{Euclid Ave. Fayette County}

The road diet was completed in 2000 and covers 1.13 miles of the highway. The main cross section has two 12-foot lanes with a 14-foot two-way left-turn lane and 7-foot bicycle lanes in both directions. The before-after crash analysis conducted showed a 56 percent overall decrease in crashes with an accompanying decrease of 77 percent in rear end and 88 percent in sideswipe crashes. There was a reduction in the ADT (from 15,900 vpd in 1996 to 13,700 vpd in 2004) along this roadway section, which is likely due to the closure of Rose Street in the vicinity of Euclid Avenue and not the result of the road diet. Examination of the crash rates indicated that the total crash rates as well as the total number of crashes was reduced indicating that the crash reduction was due to the road diet installation. The travel time studies conducted showed some congestion issues with an average travel speed of $12 \mathrm{mph}$ during the PM peak period. Overall, this road diet conversion could be considered as a successful installation.

\section{KY 1428 Floyd County}

The road diet was completed in 2005 and covers 0.93 miles of the highway. The main cross section has two 12-foot lanes with a 12-foot two-way left-turn lane in the north and two-11-foot lanes and an 11-foot two-way left tur $\mathrm{n}$ lane in the south section. The before-after crash analysis conducted showed a 55 percent overall decrease in crashes with an accompanying decrease of 17 percent in rear end, 76 percent in angle crashes, and 40 percent in sideswipe crashes. There was an increase in the ADT (from 15,939 vpd in 2005 to 16,159 vpd in 2009) along this roadway section. However, a reduction in crash rates was observed indicating that the crash reduction was due to the road diet installation. The travel time studies conducted showed no congestion issues with an average travel speed of $21 \mathrm{mph}$ during the PM peak period. Overall, this road diet conversion could be considered as a successful installation.

\section{US 172 Mercer County}

The road diet was completed in 2006 and covers 0.82 miles of the highway. The main cross section has two 15-foot lanes with a 12-foot two-way left-turn lane. The before-after crash analysis conducted showed a 41 percent overall decrease in crashes with an accompanying decrease of 23 percent in rear end crashes and 66 percent in sideswipe crashes. ADT was 
relatively stable on the corridor between 2002 (12,600 vpd ) and 2009 (11,300 vpd) Examination of the crash rates indicated that the total crash rates as well as the total number of crashes was reduced indicating that the crash reduction was due to the road diet installation. The travel time studies conducted did not demonstrate any significant congestion issues with an average travel speed of $13 \mathrm{mph}$ during the PM peak period. Overall, this road diet conversion could be considered as a successful installation.

\section{US 60 Woodford County}

The road diet was completed in 2008 and covers 0.83 miles of the highway. The main cross section has two 11-foot lanes with a 12-foot two-way left-turn lane and 5-foot bicycle lanes in both directions. Some sections of the roadway also include a 7 -foot shoulder and the conversion of the bicycle lane to parking. There was no change in the ADT of $11,00 \mathrm{vpd}$ along this roadway section. The travel time studies conducted did not demonstrate any significant congestion issues with an average travel speed of $22 \mathrm{mph}$ during the PM peak period.

The before-after crash analysis conducted showed a 25 percent overall increase in crashes with an accompanying increase of 80 percent in rear end and 50 percent in sideswipe crashes. The analysis showed that most of the after crashes were in the vicinity the US 60 Bypass and Wilson Avenue intersection, i.e. the west terminus of the project (Figure 18). The review showed that these crashes are associated with design issues of improper transition of the road diet terminus and access management at the same location. As shown below, westbound traffic in the right lane merges into the inner lane. At the same time westbound vehicles turning into the access points on the south side of the roadway will stop in the inner lane waiting to turn. Overall, this road diet conversion could be considered as a successful installation with the exception of the transition and access management issues at the west terminus of the road diet.

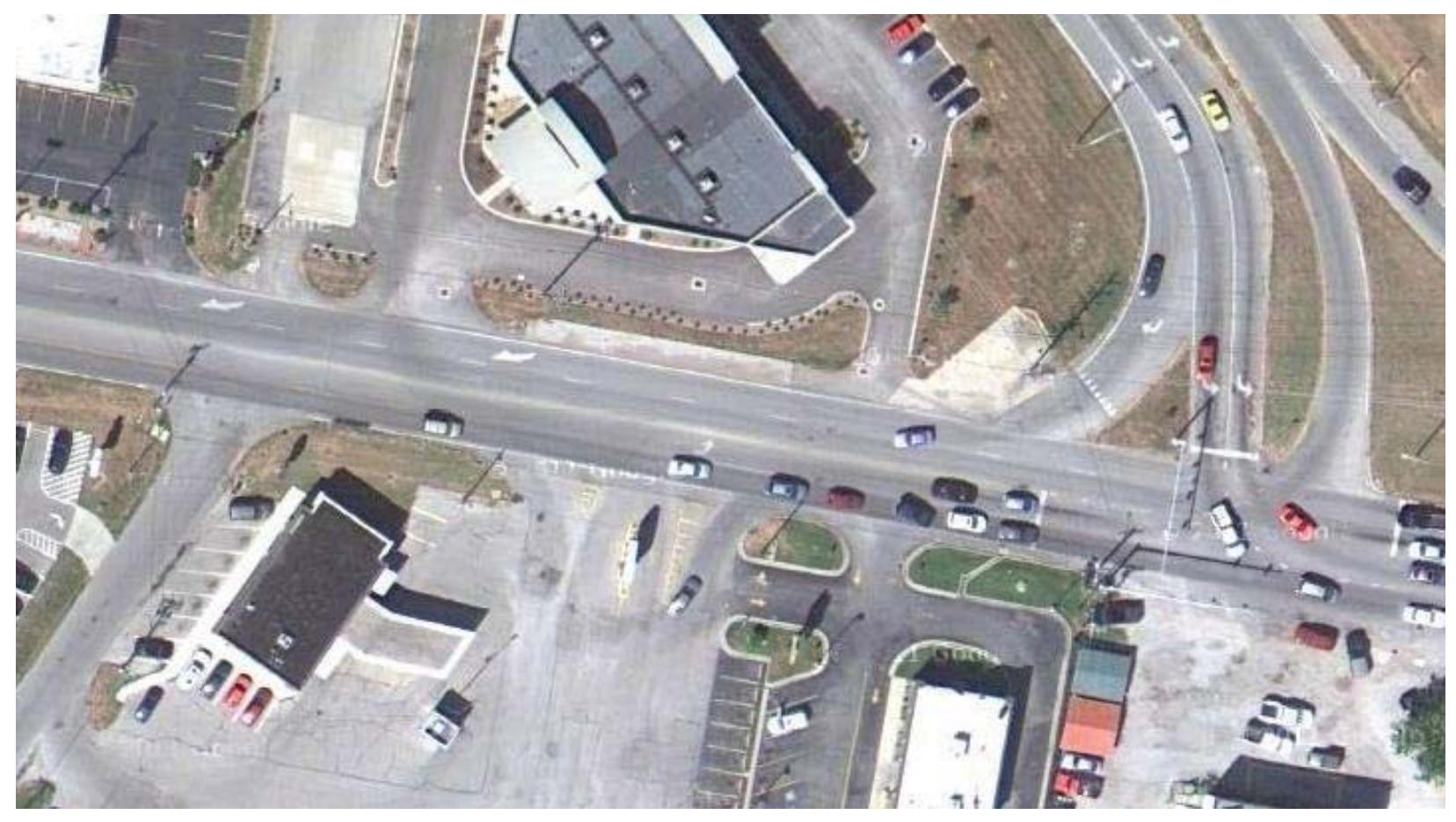

Figure 18 Western terminus of US 60 road diet 


\section{Summary}

In general, it can be concluded that the five cases evaluated are successful road diet conversions. The installations covered roadway lengths between 0.82 to 1.41 miles and a variety of conditions. The ADT ranges between 7,400 to 16,150 vpd indicate also a wide range of volumes where road diets have been implemented. In three of the four cases, safety improved significantly and sideswipe and rear end crashes showed large reductions. There were no significant congestion issues resulting from the installation and traffic volumes showed a slight decrease after the conversion.

One of the cases that showed an increase in crashes after the installation points to the need for developing proper designs for the transition into and out of the road diet. This is critical to avoid potential safety problems associated with merging of traffic and thus creating an inappropriate environment. The same case also identifies access management as an issue for review to avoid potential safety problems. 


\section{GUIDELINES}

The data analyzed here was utilized to develop guidelines for road diet conversions. The guidelines are presented in Appendix B as a standalone document and they will refer the user to this report for additional background information. A brief description of the guidelines is provided here.

\section{Road Diet Conversions}

The guidelines focus on the determination of whether a road diet application is appropriate considering operational, safety, and other factors that could have a bearing on the decision to implement a road diet conversion. To achieve this, a flow chart was developed that identifies the various steps to be taken when such decision is evaluated (Figure 19).

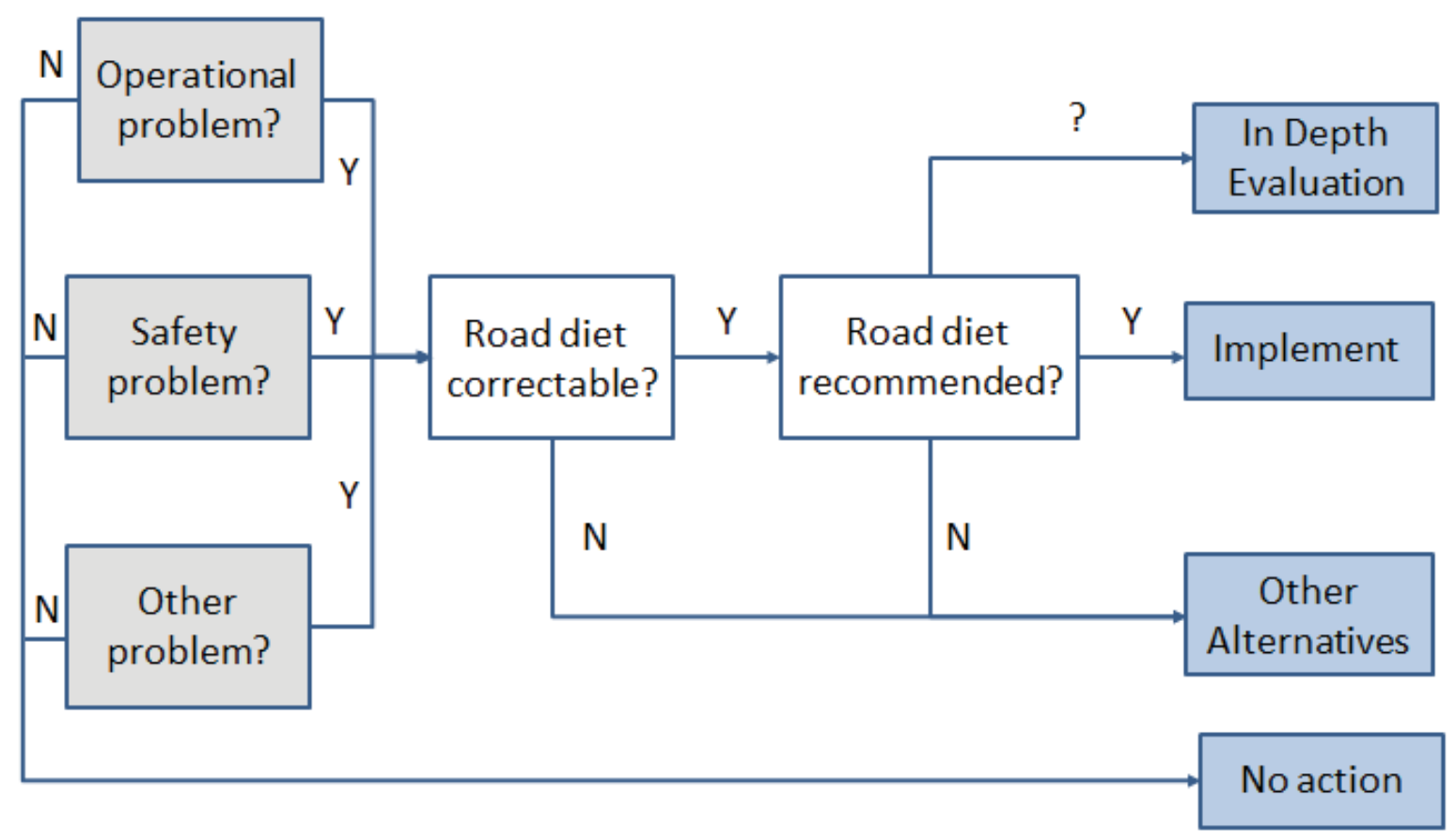

Figure 19 Decision-action flow chart for road diet evaluations

The flow chart allows the user to identify the appropriate action to be undertaken in order to determine whether the road diet will improve the operations, safety or other performance issues associated with the road. To address this, lists of possible problems correctable by a road diet implementation are identified. In addition to these lists, the required items for conducting an indepth evaluation are also identified in order to allow for a complete evaluation of the implications from a road diet implementation. 


\section{CONCLUSIONS AND SUMMARY}

This report provides design guidance on when road diet conversions are feasible alternatives and provides the required support for determining the appropriateness of road diet conversions. A structured approach was followed that considered the operational and safety evaluation of comparisons between three- and four-lane roads and identified parameters to be considered during such evaluations.

Typically, road diet conversions will operate at acceptable levels as long as the signalized intersections do not present any operational problems. Therefore, the development of the guidelines focused on evaluating and comparing the operation of three- and four-lane roads at signalized intersections. The general approach utilized a series of simulations for developing the required guidelines for road diet conversions. These simulations were performed with the CORridor SIMulation (CORSIM) and VISSIM software. Statistical analysis was employed to identify trends and develop potential ranges of feasible and un-feasible conditions as well as volume combinations where additional evaluation and analysis are needed.

An important aspect of the work completed here is the extension of the usable range of volumes where road diets could be beneficial. Past work recommended their application for roads with an ADT up to $17,000 \mathrm{vpd}$. The current research indicates that such conversions could work for roads with significantly greater volumes, up to $23,000 \mathrm{vpd}$. Moreover, the findings here identify the effect of the side street volume, indicating that both volumes need to be considered when determining whether such a conversion should be considered.

Safety analysis was also completed using the micro-simulation results from VISSIM and processing the trajectory files with the SSAM. SSAM then provided an estimate of rear end and lane change conflicts for both the three-lane and four-lane sections. Analysis indicated that at signalized intersections with moderate left-turn percentages, the road diet decreased the potential for rear end conflicts, but when low or very high left-turn percentages were present, the four-lane section performed better. When examining lane change conflicts, both the three- and four-lane sections had low frequencies of conflicts at low volumes, but at higher volumes, the three-lane section maintained low overall conflicts, while the 4-lane saw a significant increase in conflicts.

Typical considerations for road diet conversions include traffic volumes of the minor and main streets, main street left-turn percentages, functional classification, access frequency and land use. Among these factors, functional classification is an important consideration but has limited effect on the decision of a road diet conversion. The roadway classification is meaningful for a variety of aspects, but the traffic volumes along the roadway are more critical than its classification. There is a wide range of volumes for roadways that are classified within a single category practically rendering this factor unusable for consideration in road diet conversions. The effect of access points is also important in determining operational efficiency and performance of a roadway. However, the existing cases reviewed along with some of the proposed cases evaluated indicated that there are great differences in access frequencies and this factor has a minor influence on the operational performance of the road diet (although it has been observed that a road diet cross-section can facilitate left-turn egress from access points). Finally, land use along the corridors reviewed was mixed with predominantly residential and commercial uses and had no discernable effect on the road diet performance. Given these considerations, the factors utilized here include traffic volumes and left-turn percentages as predictors for the operational performance of the road diet conversion. However, the other 
factors noted here need to be considered when determining the overall feasibility of a road diet as the final checklist presented later indicates.

Five road diet conversions in Kentucky were identified and evaluated to determine their overall effects and identify elements that could also assist in the development of the guidelines. In general, it was concluded that the four cases evaluated are successful road diet conversions. The installations covered roadway lengths from 0.82 to 1.41 with ADT ranges from 7,400 to 13,700 vehicles per day indicating also a wide range of conditions where road diets have been implemented. In three of the four cases, safety improved significantly and sideswipe and rear end crashes showed large reductions. There were no significant congestion issues resulting from the road diets. One of the cases did show an increase in crashes after the installation, but further analysis of the crash patterns identified the need for developing proper transitions into the road diet. The transition design is critical to avoid potential safety problems associated with merging of traffic and thus creating an inappropriate environment. The same case also identifies access management as an issue for review to avoid potential safety problems.

Road diets may also improve the operational efficiency of signalized and unsignalized intersections on a corridor. Operational improvements are typically seen when high ADTs and high left-turn percentages are present on the primary and cross-street. Typically road diets are shown to have reduced delays and reduced queues when high left-turn percentages AND high volumes are present. 


\section{REFERENCES}

Burden, D., and Lagerwey, P. (1999) Road Diets: Fixing the Big Roads. www.walkable.org/download/rdiets.pdf. Accessed July 10, 2010.

Federal Highway Administration (2004) Evaluation of Lane Reduction "Road Diet" Measures and Their Effects of Crashes and Injuries. FHWA-HRT-04-082, Washington, D.C.

Federal Highway Administration (2010) Evaluation of Lane Reduction Road Diet Measures on Crashes. FHWA-HRT-10-053, Washington, D.C.

Federal Highway Administration (2009) Manual on Uniform Traffic Control Devices Washington, D.C.

Gates,T. Noyce, D., Talada, V., Hill, L (2007) The Safety and Operational Effects of "Road Diet" Conversions in Minnesota. Paper No. 07-1918. Accessed from TRB 2007 Annual Meeting CDROM, Washington, D.C.

Geedipaly, S., Patil, S. and Lord, D. (2010) Examining Methods for Estimating Crash Counts According to Their Collision Type Transportation Research Record 2165, Washington, D.C., pp. 12-20.

Gettman, D., Pu, L., Tarek, S., and Shelby, S. (2008) Surrogate Safety Assessment Model and Validation: Final Report, FHWA-HRT-08-051, Washington, D.C.

Huang, H., Stewart, J., and Zegeer, C. (2002) Evaluation of Lane Reduction „Road Diet" Measures on Crashes and Injuries. Transportation Research Record 1784, Washington, DC, pp. 80-90.

Knapp, K., and Giese, K. (2001) Guidelines for the Conversion of Urban Four-Lane Undivided Roadways to Three-Lane Two-Way Left-Turn Lane Facilities Final Report. Center for Transportation Research and Education, lowa State University, Ames, IA.

Kentucky Transportation Cabinet (2009) Design Memo 03-09 Manual, Frankfort, KY

Messer, C and Fambro, D. (1977). Critical lane Analysis for Intersection Design. Transportation Research Record 644, Transportation Research Board. Washington, D.C. pp. 26-35.

Pawlovich, M., Li, W., Carriquiry, A., and Welch, T. (2006) lowa"s Experience with "Road Diet" Measures: Impacts on Crash Frequencies and Crash Rates Assessed Following a Bayesian Approach. Journal of the Transportation Research Board No. 1953, Washington, D.C., pp. 163171.

Rosales, J. (2006) Road Diet handbook: Setting Trends for Livable Communities, Second Editions, ITE Washington, D.C.

Rosales, and Knapp, K. (2005) Livability Impacts of Geometric Design Cross-Section Changes of Road Diets. Proceedings 3rd International Symposium on Highway Geometric Design, Chicago, IL. 
Transportation Research Board (1985) Highway Capacity Manual TRB Special Report 209, Washington, D.C.

Welch, T. (1999) The Conversion of Four-Lane Undivided Urban Roadways to Three-Lane Facilities. Presented at the Transportation Research Board Urban Street Symposium, Dallas, TX. 
APPENDIX A

Kentucky Case Studies 


\section{ROAD DIET CASE STUDY EVALUATION US 60 VERSAILLES, KY \\ (FINAL) REPORT}

Prepared by

Kentucky Transportation Center

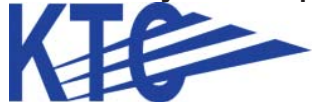

\section{Prepared for:}

Kentucky Transportation Cabinet Division of Planning

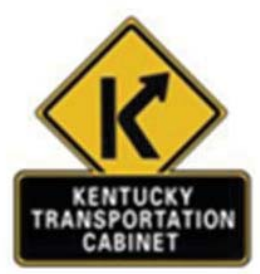

Date:

August 2011 


\section{TABLE OF CONTENTS}

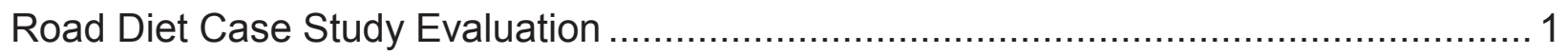

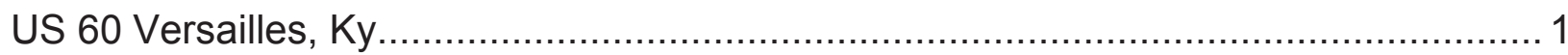

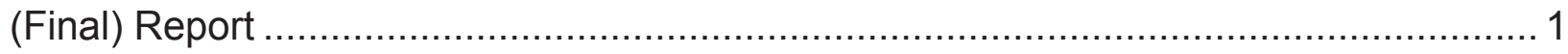

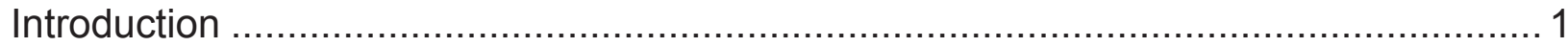

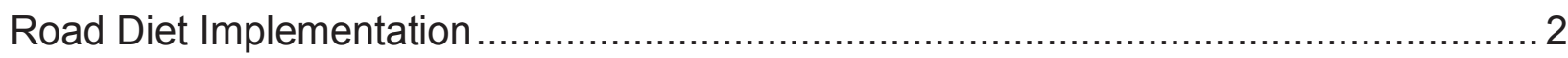

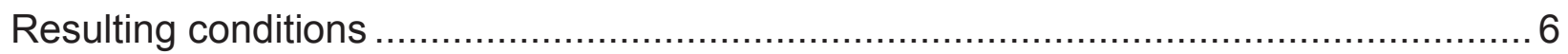

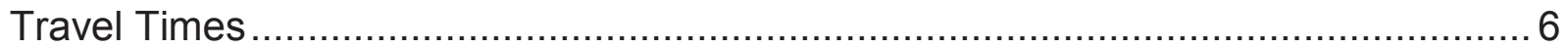

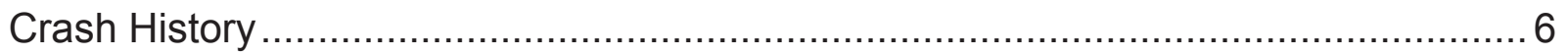

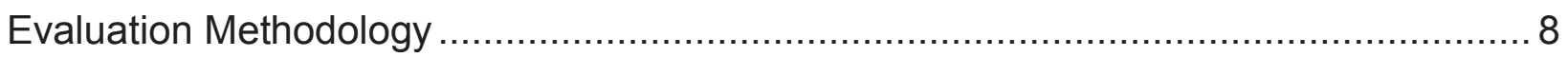

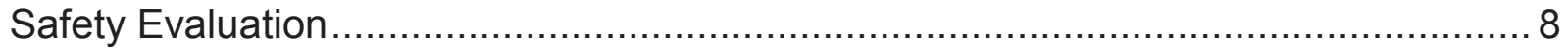

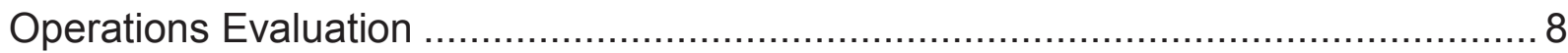

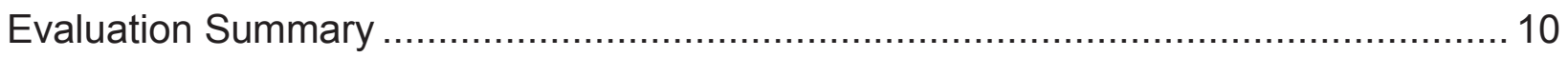

\section{LIST OF FIGURES}

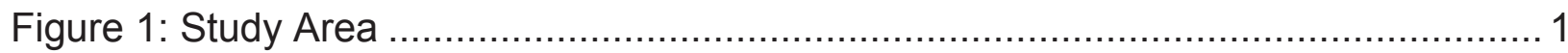

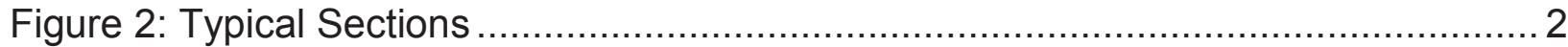

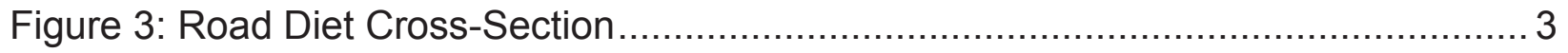

Figure 4: US 60 with Standard Road Diet …................................................... 3

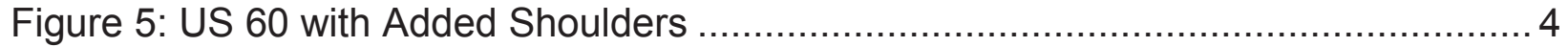

Figure 6: US 60 with Parking........................................................................... 4

Figure 7: US 60 Road Diet Types and Locations .................................................. 5

\section{LIST OF TABLES}

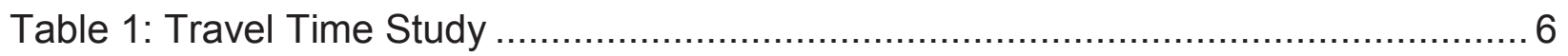

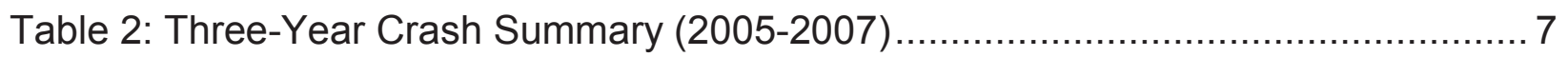

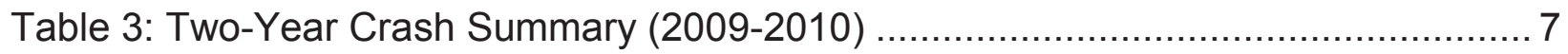

Table 4: Summary of Road Diet Effects ............................................................... 9 


\section{INTRODUCTION}

The purpose of this report is to evaluate and present the effects associated with the conversion of a section of US 60 in Versailles, Woodford County KY. This section underwent a "road diet" in 2008 in which the road was reduced from a 4-lane facility to a 3-lane facility. The area considered in this study extends on US 60 from Main Street to the US 60 Bypass (MP 0.960 to MP 1.790) for a distance of .83 miles. The location of the study area is shown below in Figure 1.

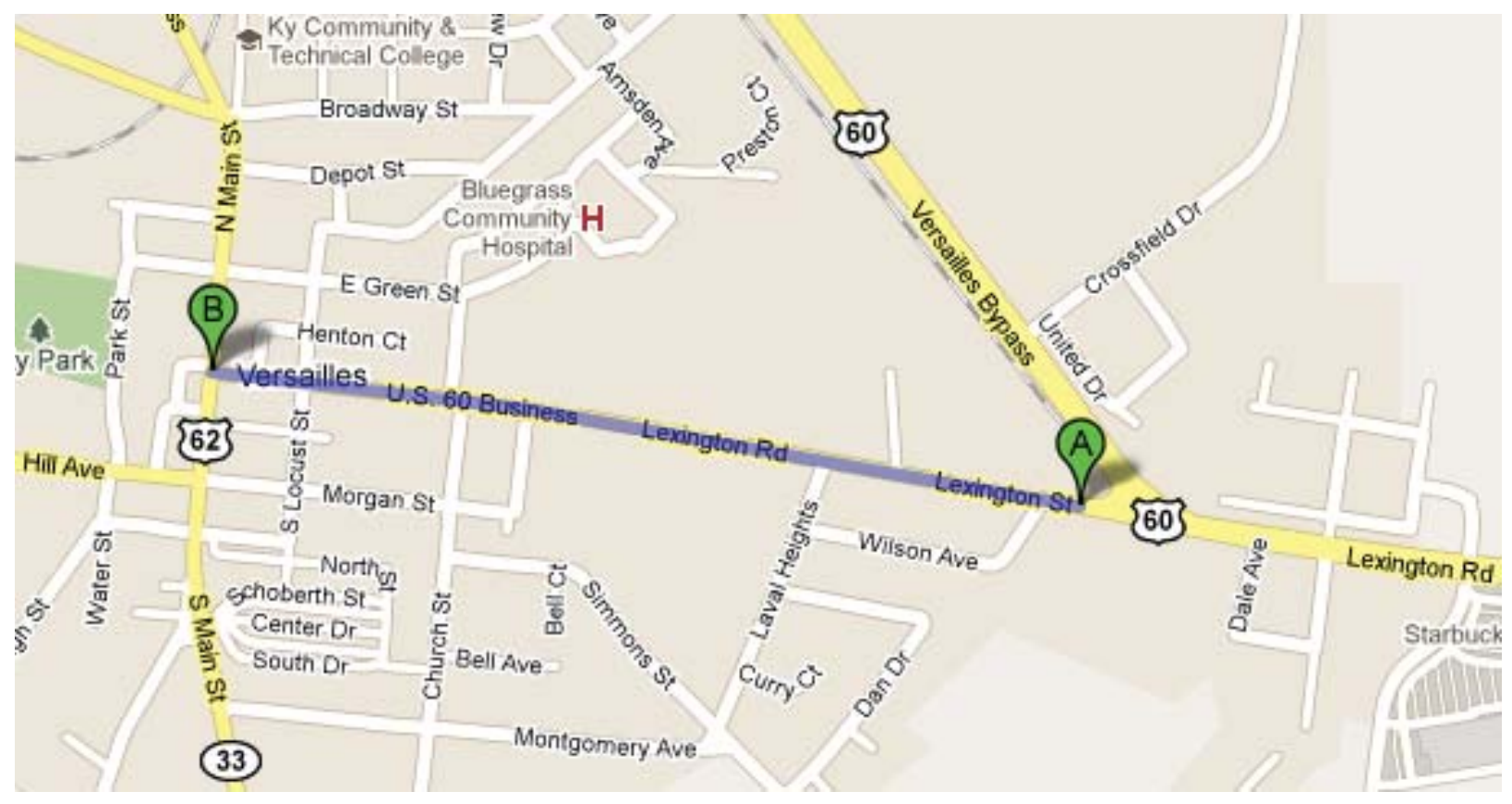

Figure 1: Study Area

This report summarizes the results of the road diet on this corridor while concentrating on aspects such as travel times, crash records, and safety. As well as resulting conditions, former conditions will be discussed. 


\section{ROAD DIET IMPLEMENTATION}

The study section was originally four or five lanes wide, each about $11 \mathrm{ft}$ in width, with two lanes in each direction, and sometimes contained a median. Typical cross sections are shown in Figure 2. It should also be noted that along the .8 mile section there are about 75 residential or commercial entrances/exits. Furthermore, there are 3 signalized intersections: at Main Street, Locust, and the US60 bypass. The frequency of access points indicates that there are several left turns along the corridor. This resulted in frequent backed up traffic in the inside lanes in each direction resulting in the use of these lanes as exclusive left-turn lanes at peak conditions. There is also the potential for unsafe conditions due to the need of through vehicles to avoid the blocked lanes.
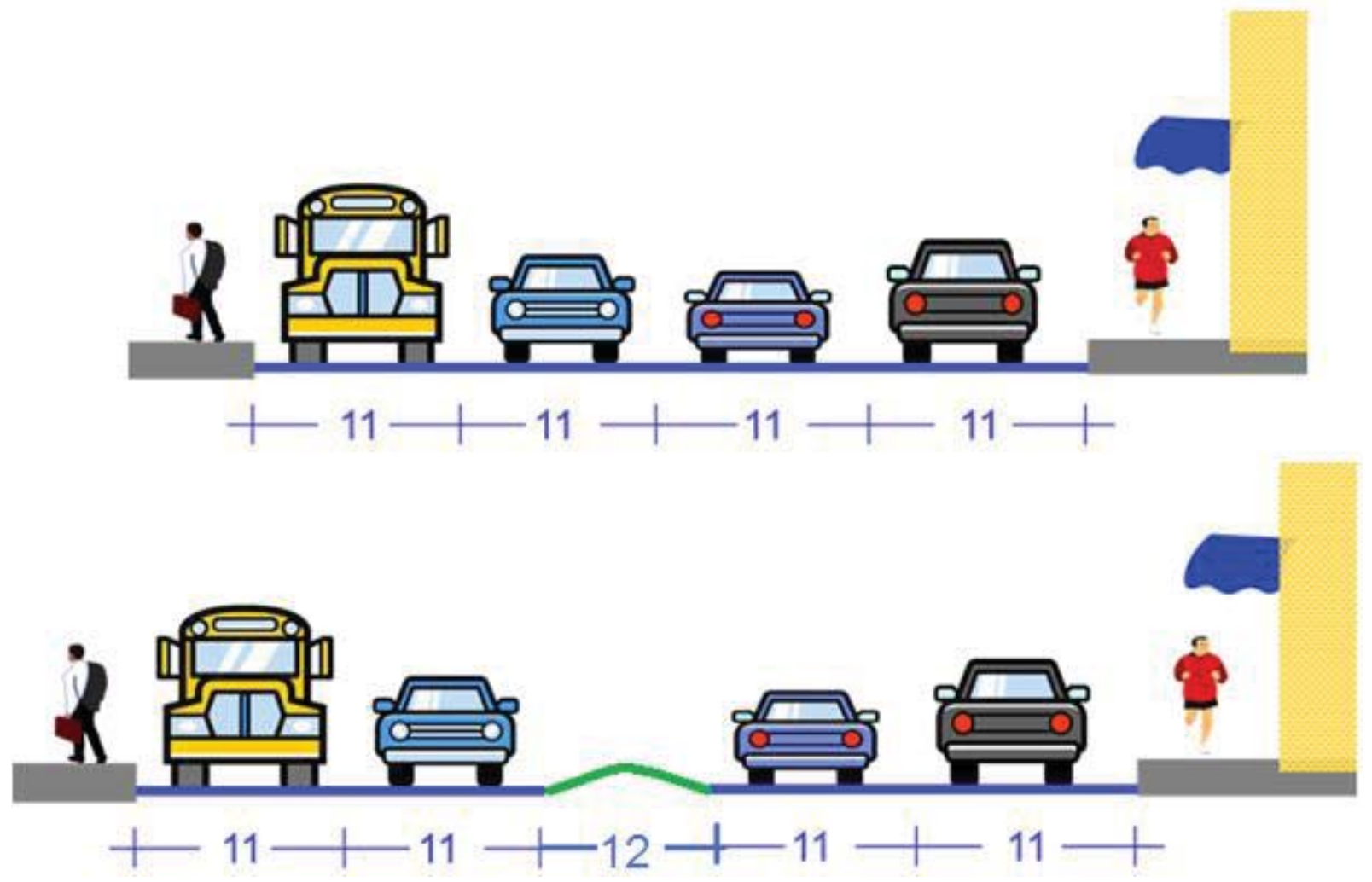

Figure 2: Typical Sections

The road diet approach distributed the available roadway width differently to address these issues. It should be noted that there was not a uniform cross section for the entire corridor. The most typical cross section creates only one 11-foot lane in each direction while a 12-foot two-way-left-turn lane (TWLTL) was added in the center. Additionally, 5foot bike lanes were added on each side in hopes of increasing multimodal transportation. An example of this cross-section can be seen in Figure 3 and a constructed example is shown in Figure 4. 


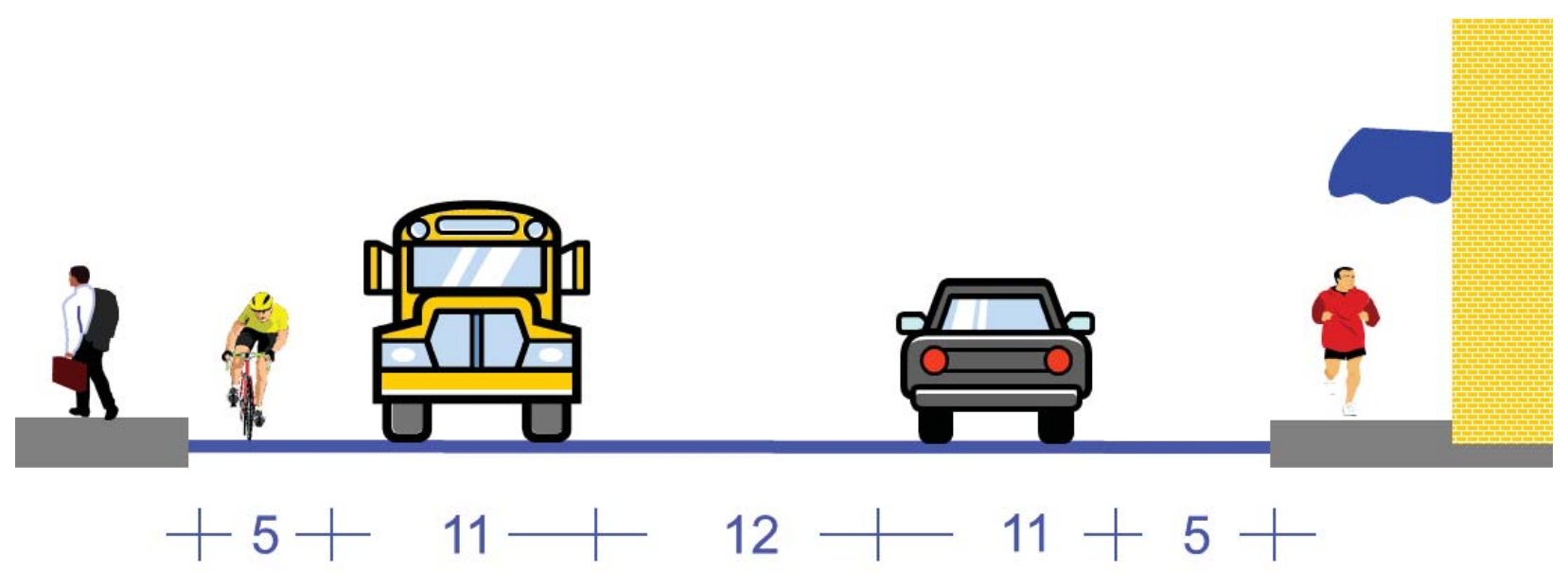

Figure 3: Road Diet Cross-Section

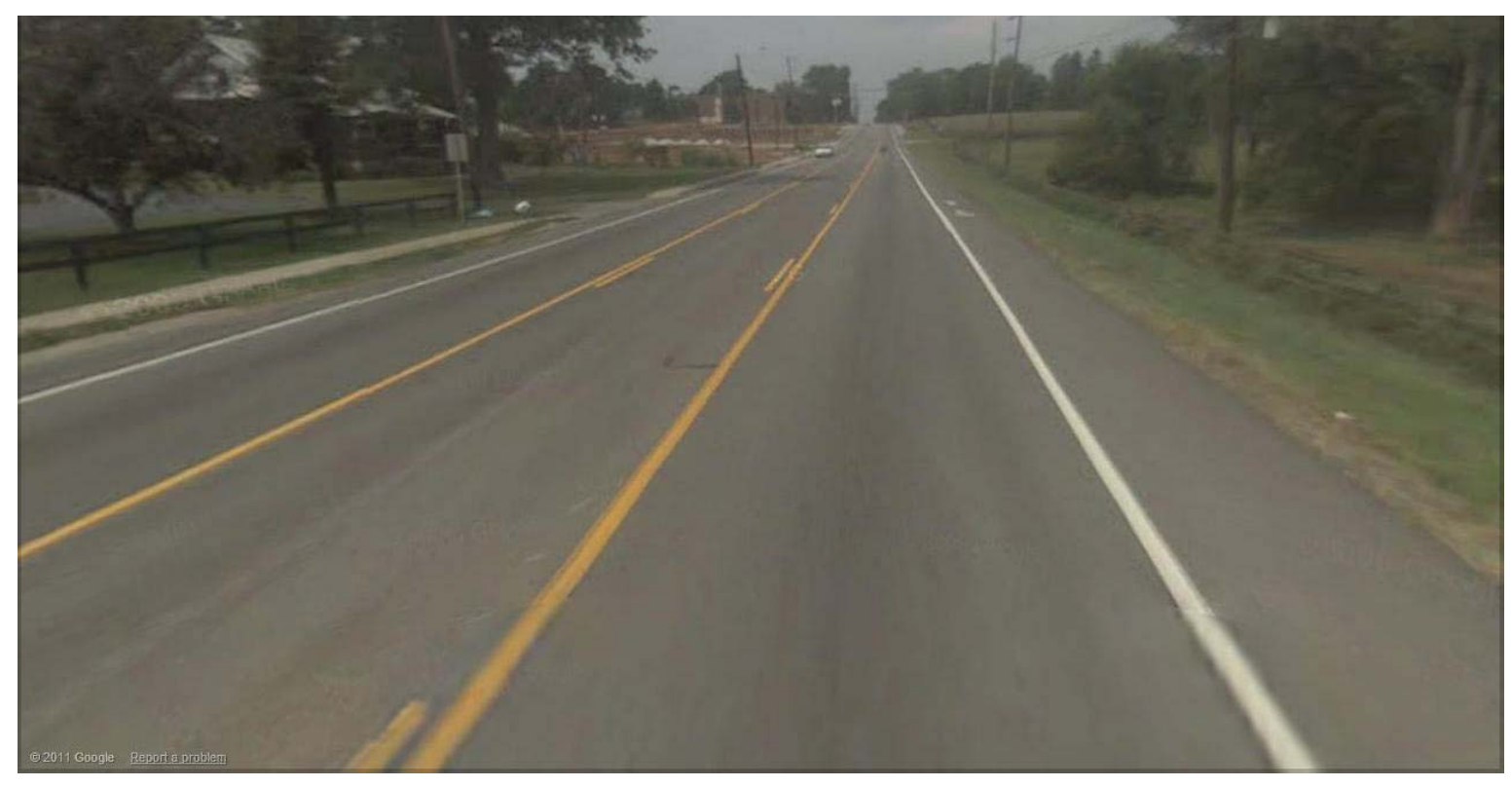

Figure 4: US 60 with Standard Road Diet

Another option similar to the one described above adds 7-foot shoulders to each side. This was done where there was a median previously, fewer access points were present, and a wider paved surface existed. An example of this can be seen in Figure 5. 


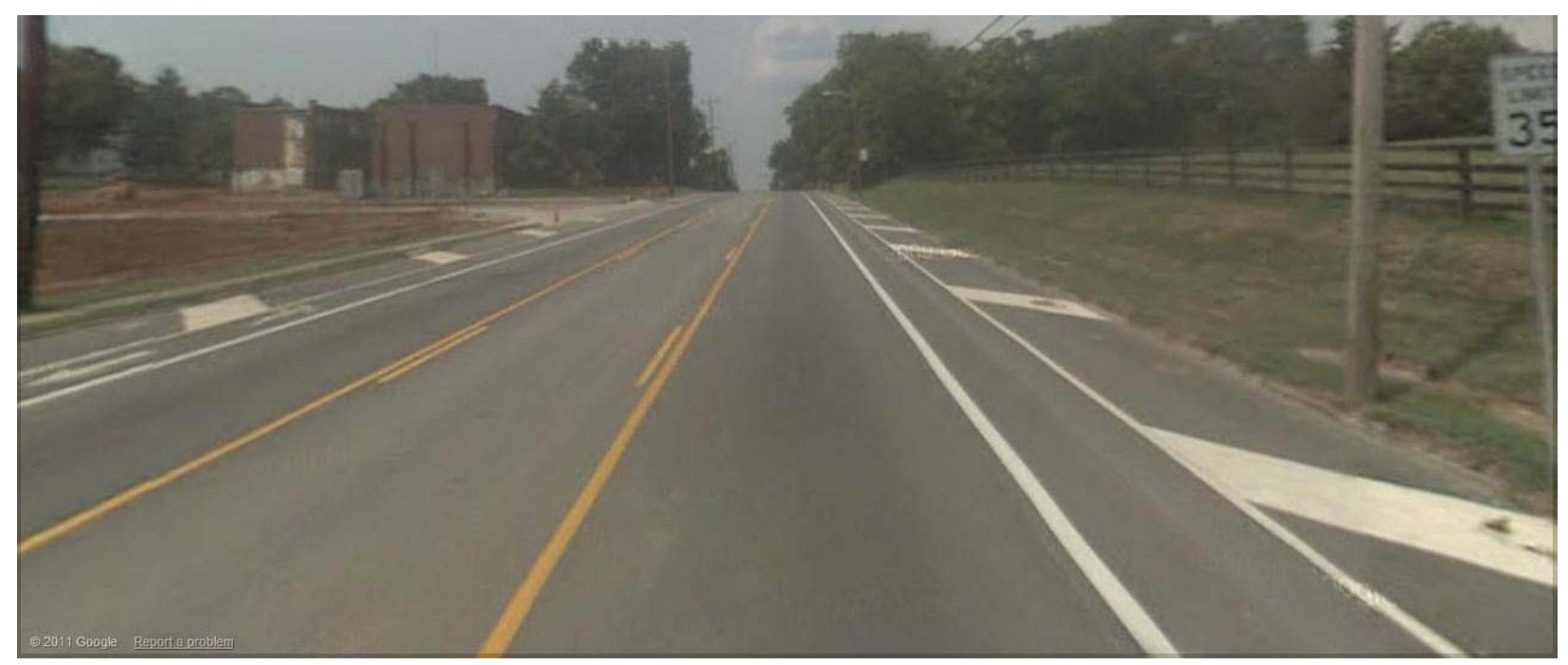

\section{Figure 5: US 60 with Added Shoulders}

Finally, a third cross section was utilized where the bike lanes were removed to provide for parking spaces. This cross section is utilized in the section in the vicinity of the downtown area to create more parking. An example of this can be seen in Figure 6 below. Figure 7 summarizes the use of each cross section along the corridor.

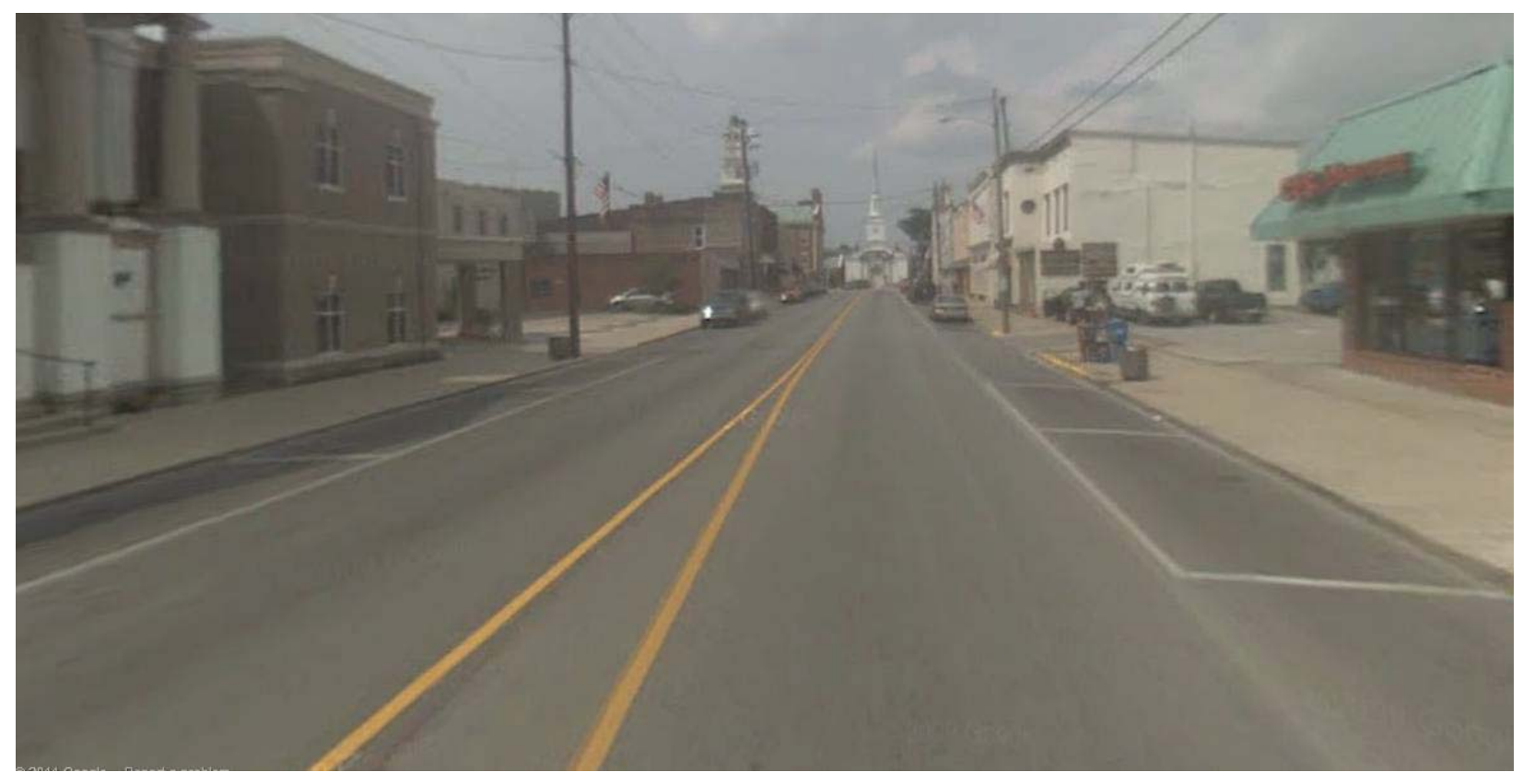

Figure 6: US 60 with Parking 


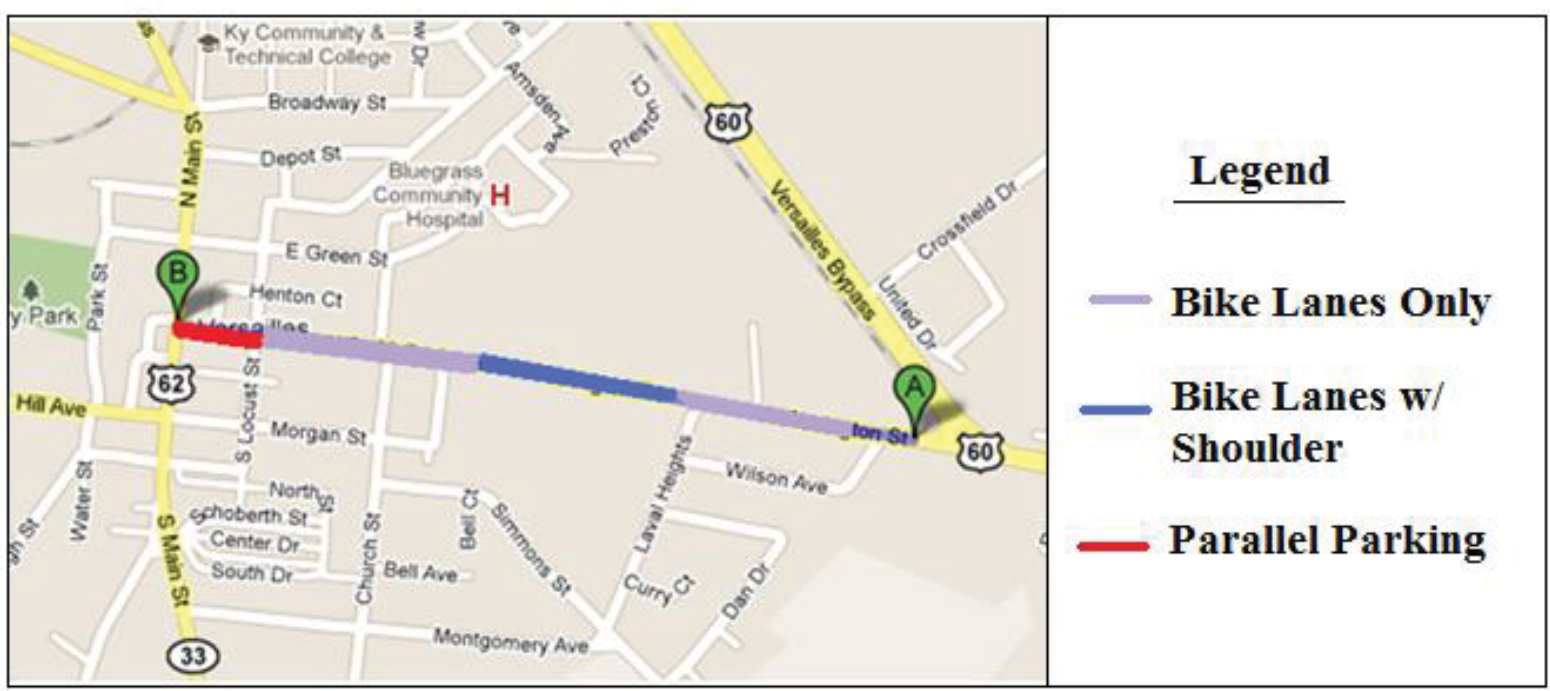

Figure 7: US 60 Road Diet Types and Locations

One of the main goals of the road diet was to improve safety along with improving efficiency. The two-way left-turn lane provides motorists with a refuge area and removes them form the traffic while waiting to complete their turns. 


\section{RESULTING CONDITIONS}

This section contains the results concerning travel times and crash history.

\section{Travel Times}

Travel times were collected along the corridor to determine average travel times as well as average speeds for peak travel. Travel times for each direction are shown in Table 1. The travel times were calculated based on 5 runs in each direction.

Table 1: Travel Time Study

\begin{tabular}{ccc}
\hline & \multicolumn{2}{c}{ Travel Time (Seconds) } \\
\cline { 2 - 3 } Run & West & East \\
\hline 1 & 130 & 130 \\
2 & 120 & 90 \\
3 & 140 & 150 \\
4 & 160 & 140 \\
5 & 120 & 120 \\
\hline Average & 134 & 126 \\
\hline
\end{tabular}

The average westbound travel time was 134 seconds (or 2.23 minutes) and the average eastbound travel time was 126 seconds (or 2.1 minutes). This means the combined average is 130 seconds (or 2.17 minutes), thus, the average directional trip speed is 23 miles per hour. It should be noted that the existing speed limit is 35 miles per hour.

\section{Crash History}

A three-year crash history was collected and analyzed along the corridor. The period evaluated was between January 1, 2005 and December 31, 2007 and there were 39 crashes recorded in this period. Table 2 summarizes the crashes by type and location. Crashes are disaggregated by signalized and midblock crashes to assist in identifying crash patterns that may be affected by the implementation of the road diet. Table 3 summarizes crashes for the two years following the road diet change and the date is similarly arranged as in Table 2. The dates of the crashes evaluated extend from January 1, 2009 to December 31, 2010. 
Table 2: Pre- Road Diet Three-Year Crash Summary (2005-2007)

\begin{tabular}{|c|c|c|c|}
\hline Crash Type & Total & Signalized & Midblock \\
\hline Head On & 3 & 2 & 1 \\
\hline Backing & 1 & 0 & 1 \\
\hline Opposing left turn & 3 & 3 & 0 \\
\hline Rear end & 8 & 3 & 5 \\
\hline Sideswipe-Opposite direction & 0 & 0 & 0 \\
\hline Sideswipe- Same direction & 3 & 1 & 2 \\
\hline Single vehicle & 1 & 0 & 1 \\
\hline Pedestrian-Bicyclist & 0 & 0 & 0 \\
\hline Other & 20 & 6 & 14 \\
\hline Total & 39 & 15 & 24 \\
\hline
\end{tabular}

Table 3: Post- Road Diet Three-Year Crash Summary (2009-2010)

\begin{tabular}{|c|c|c|c|}
\hline Crash Type & Total & Signalized & Midblock \\
\hline Head On & 1 & 1 & 0 \\
\hline Backing & 5 & 2 & 3 \\
\hline Opposing left turn & 5 & 5 & 0 \\
\hline Rear end & 18 & 4 & 14 \\
\hline Sideswipe-Opposite direction & 1 & 0 & 1 \\
\hline Sideswipe- Same direction & 4 & 0 & 4 \\
\hline Single vehicle & 1 & 0 & 1 \\
\hline Pedestrian-Bicyclist & 0 & 0 & 0 \\
\hline Other & 14 & 4 & 10 \\
\hline Total & 49 & 16 & 33 \\
\hline
\end{tabular}

The data in Tables 3 and 4 indicate that there is an overall increase in crashes for the after the installation period. This difference is even greater when considering that the after period is only for two years. In this case, the average number of crashes before is 13 per year while after the road diet the number of crashes is 24.5 per year. This is an $88 \%$ increase. 


\section{EVALUATION METHODOLOGY}

Safety and operational improvements are typically the reasons for a road diet implementation. Therefore, the evaluation should consider metrics that could allow for comparisons of the before and after conditions as well determine the level of performance for the current conditions. These measures include crash changes and travel times and speeds.

\section{Safety Evaluation}

The comparison of the before and after the road diet periods showed that traffic crashes almost doubled per year. The data shows that crashes increased $88 \%$ per year and rear end crashes increased $225 \%$ per year. The anticipated pattern is different than the observed, i.e. rear ends and overall number of crashes are expected to decrease. An examination of the injury crashes showed a $10 \%$ reduction, indicating that a smaller percentage of crashes are injuries.

A detailed examination of the crashes along the corridor revealed that all rear end crashes in the after period were at the vicinity of the US 60 Bypass and Wilson Avenue intersection. The analysis showed that most of the westbound crashes (i.e. towards Versailles) were caused from motorists stopping to allow other motorists in the right lane to merge as the lane ended, i.e. at the transition to the road diet. In the eastbound direction, most crashes were cause by a speed differential and occupancy between the available lanes. The left lane seemed to be less occupied than the right lane, which typically will demonstrate longer queues resulting in rear end crashes. Moreover, many vehicles exiting the adjacent parking lots and making left turns were also involved in crashes with vehicles traveling eastbound. This area accounts for $35 \%$ of total crashes post-road diet while before it was only $15 \%$ of total crashes.

It was also observed that ADT remained virtually unchanged. In 2005, the volume was 10,900 vehicles per day and in 200911,000 vehicles per day. Therefore the increase in crashes cannot be attributed to the changes in ADT. The crash rates also showed a similar increase of $87 \%$ over the same period (average crash rate of 331 crashes/100 million vehicle-miles traveled per year in before period and 618 crashes/100 million vehicle-miles traveled per year in the after period).

Another important aspect is the changes in the crashes for pedestrians and bicyclists. Even though the before and after the road diet implementation data showed no crashes, this could be indicative either of lack of any such users or a safe environment for them. It is more likely that there is only a small number of users that use the facility and efforts to increase and promote its use may be needed.

\section{Operations Evaluation}

There is no travel time data available for the before period to allow for a before-after comparison. The current speeds are below the speed limit indicating that there may be some delays during the peak period. The presence of the signalized intersections could 
induce operational delays which could explain the increased travel time. However, the increased travel times are small and no congestion was observed during the data collection indicating acceptable operation. 
EVALUATION SUMMARY

The analysis shown here helps one to see the benefits and disadvantages of the road diet, allowing them to decide if it was the correct decision. Table 4 presents a summary of the evaluation parameters considered.

Table 4: Summary of Road Diet Installation Effects

\begin{tabular}{|l|l|}
\hline \multicolumn{1}{|c|}{ Aspect } & \multicolumn{1}{c|}{ Effect } \\
\hline Travel times and & Successful, possibly improved \\
\hline Intersection delays & $\begin{array}{l}\text { Declined; } \\
\text { reduced conflict points; } \\
\text { increased crashes, especially rear end; } \\
\text { reduced crashes with injuries; }\end{array}$ \\
\hline Overall safety & Most likely improved uniformity; traffic calming potential \\
\hline Pravel speeds & No Change \\
\hline Multimodal operations & No Change, encourages bicyclists more \\
\hline Access management & Improved potential \\
\hline
\end{tabular}

The travel times show an improvement overall for this road diet installation. The increase in the number of crashes is directly attributed to the lack of proper design for the transition of the existing cross section to the road diet cross section in the east end of the project. Moreover, the lack of any access management in the south side of the project contributes to the higher number of crashes in the after period. It should be noted that if the east end of the project is excluded, the safety performance of the remaining corridor is as typically anticipated, i.e. there is an overall reduction in crashes and an improvement in safety. Other aspects associated with most road diets such as improved traffic calming, improved access management potential, and improved multimodal operations could also be viewed as additional benefits for this corridor. The safety performance of this corridor points to the need for developing proper designs for the transition into and out of the road diet and therefore, a review of this may be needed. 


\section{ROAD DIET CASE STUDY EVALUATION EUCLID AVE. LEXINGTON, KY}

(FINAL) REPORT

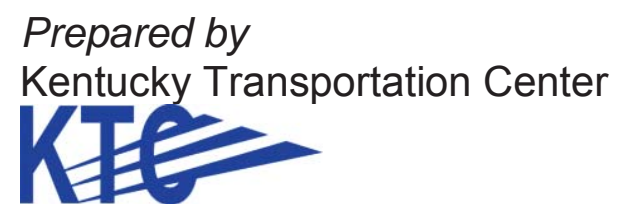

\section{Prepared for:}

Kentucky Transportation Cabinet Division of Planning

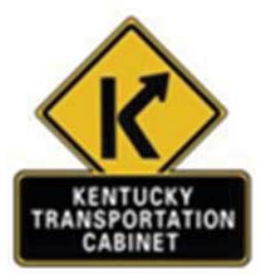

Date:

August 2011 


\section{TABLE OF CONTENTS}

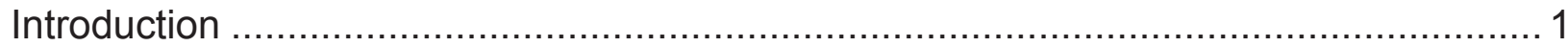

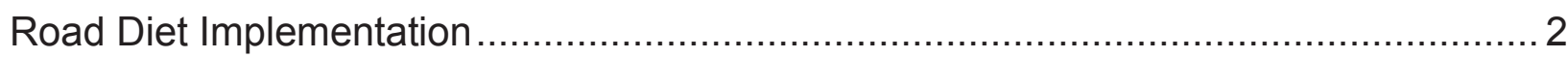

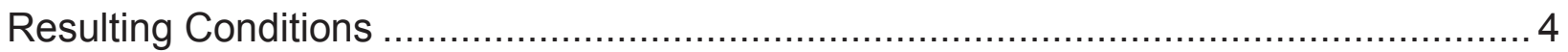

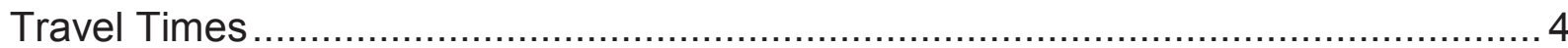

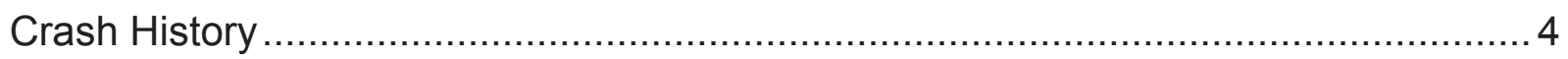

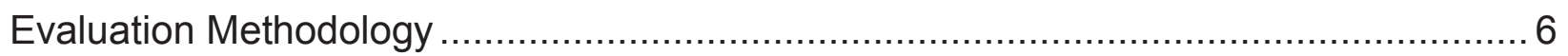

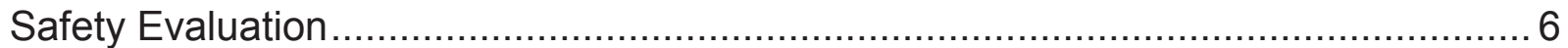

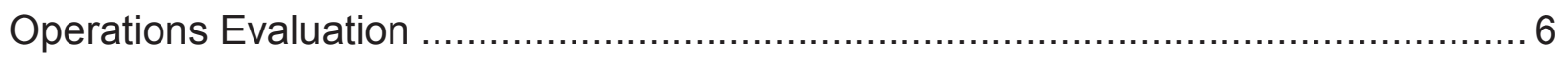

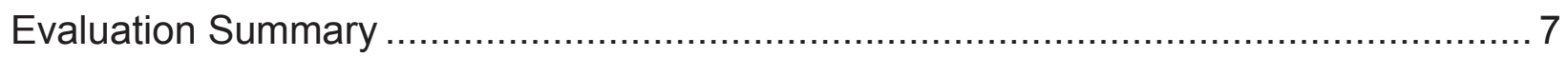

\section{LIST OF FIGURES}

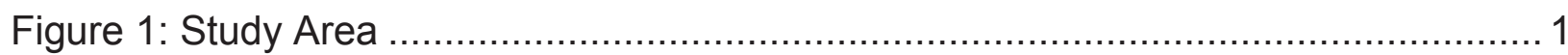

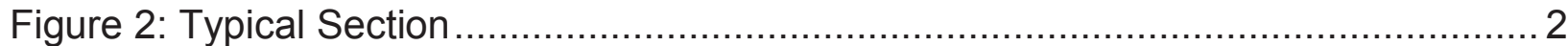

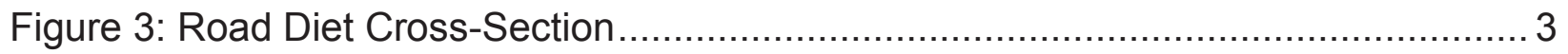

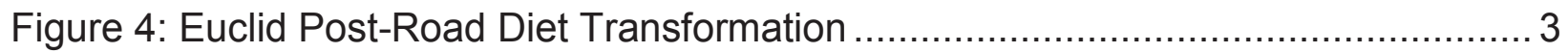

\section{LIST OF TABLES}

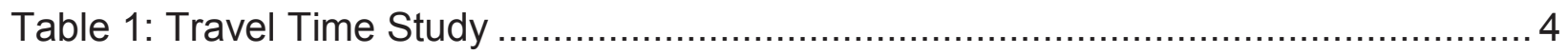

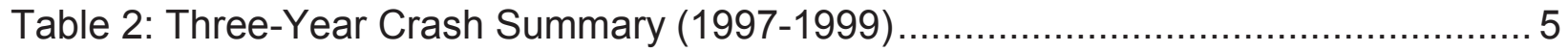

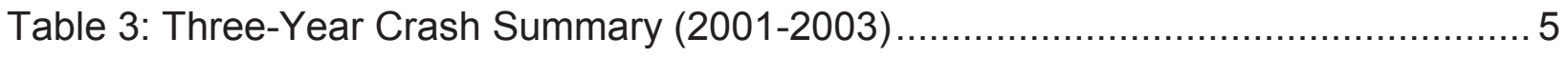

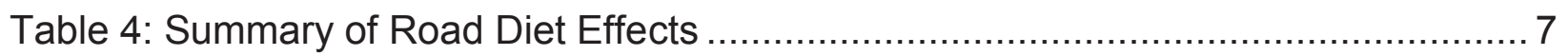




\section{INTRODUCTION}

The purpose of this report is to evaluate and present the effects associated with the conversion of a section of Euclid Avenue in Lexington, Fayette County KY. This section underwent a "road diet" in 2000 in which the road was reduced from a 4-lane facility to a 3-lane facility. The area considered in this study extends on Euclid Avenue from Ashland Avenue to S. Limestone Street (MP 12.125 to MP 13.255) for a distance a of 1.13 miles. The location of the study area is shown below in Figure 1.

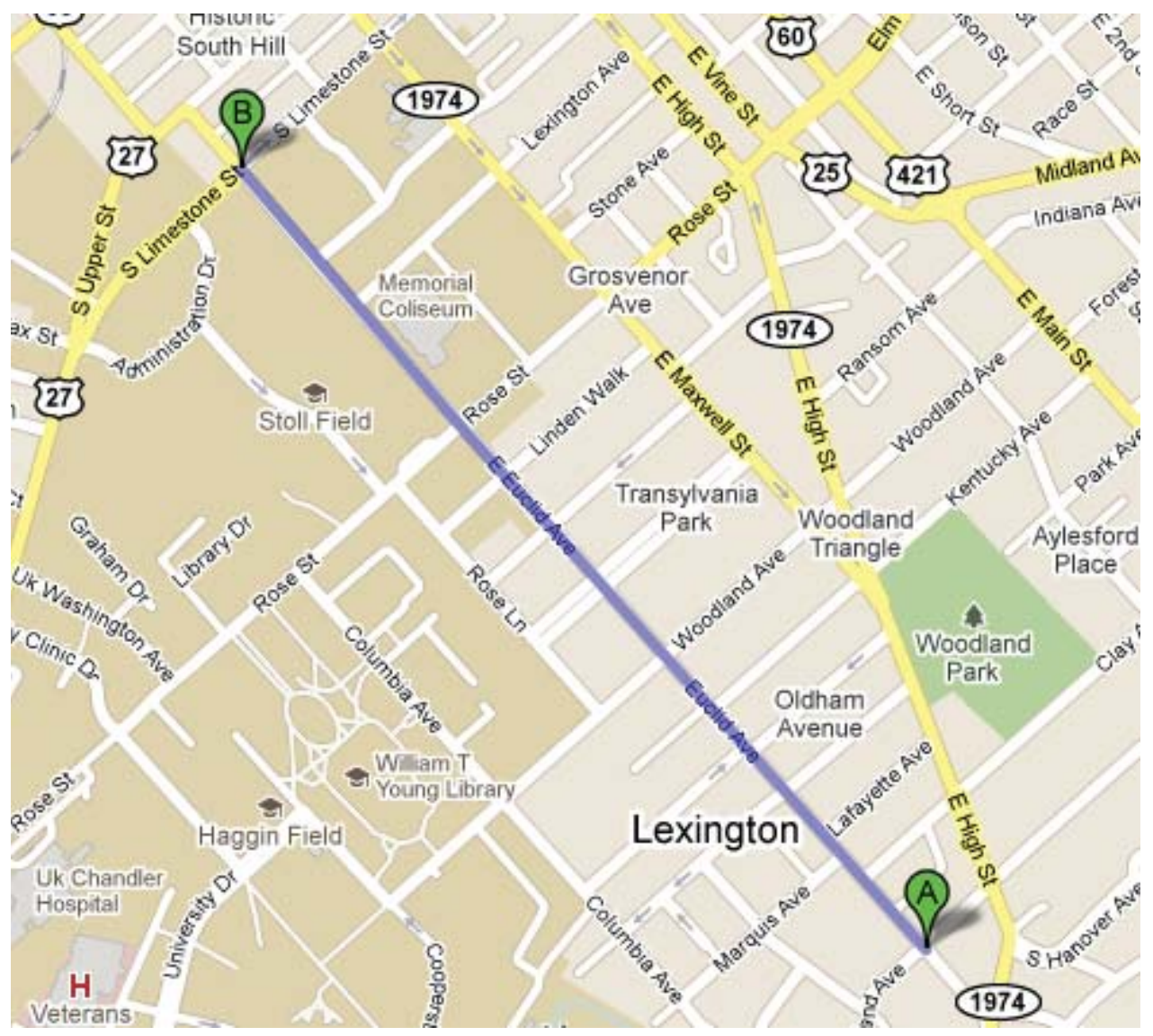

Figure 1: Study Area

This report summarizes the results of the road diet on Euclid while concentrating on aspects such as travel times, crash records, and safety. As well as resulting conditions, former conditions will be discussed. 


\section{ROAD DIET IMPLEMENTATION}

The study section was originally four lanes wide, each about $13 \mathrm{ft}$ in width, with two lanes in each direction. This cross section is shown in Figure 2. It should also be noted that along the 1.13 mile section there are 51 residential or commercial entrances/exits. Furthermore, there are five intersections with traffic signals at Limestone Street, Martin Luther King Boulevard, Rose Street, Woodland Avenue, and Ashland Avenue. The frequency of access points indicates that there are several left turns along the corridor. This resulted in frequent backed up traffic in the inside lanes in each direction resulting in the use of these lanes as exclusive left-turn lanes at peak conditions. There is also the potential for unsafe conditions due to the need of through vehicles to avoid the blocked lanes.

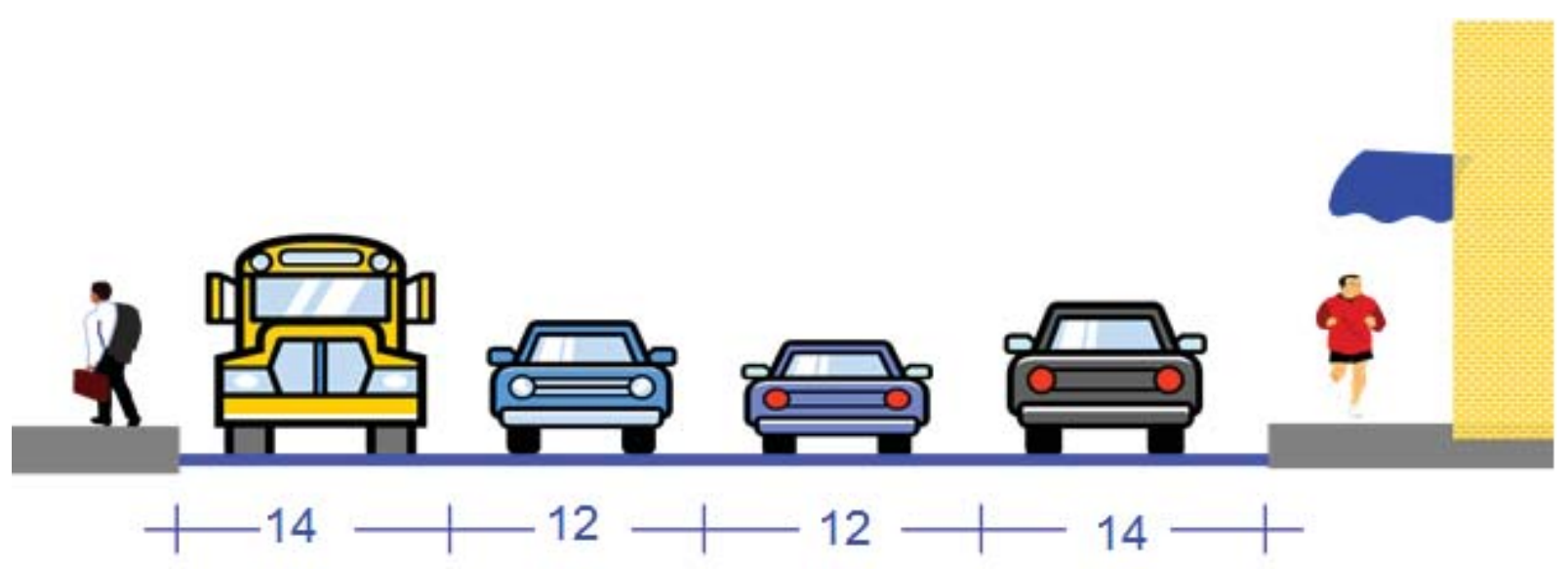

Figure 2: Typical Section

For Euclid, rear end crashes were becoming a problem as was congestion. A road diet was implemented to address these issues. A significant number of pedestrians and cyclist was also present due to the proximity of the road to the University of Kentucky campus. This solution utilized the existing paved roadway and re-divided it so that only one 12-foot lane remained in each direction while a 14-foot two-way-left-turn lane was added in the center. Additionally, 7-foot bike lanes were added on each side aiming to increase multimodal transportation. An example of this cross-section can be seen in Figure 3. 


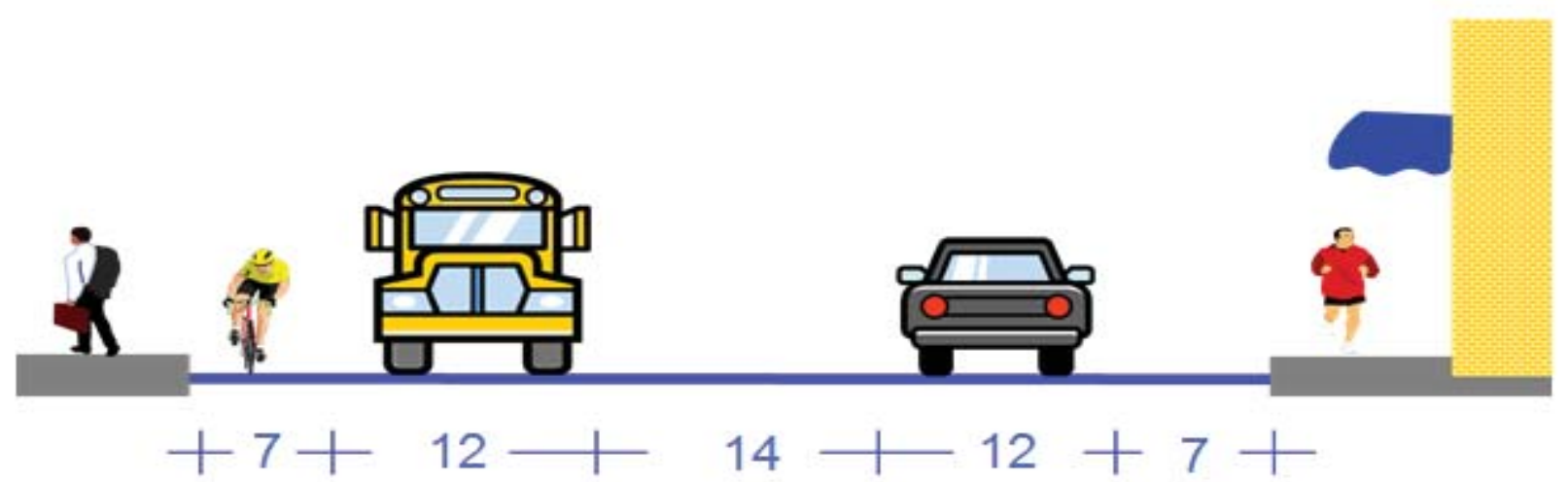

Figure 3: Road Diet Cross-Section

One of the main goals of the road diet was to improve safety along with improving efficiency. The two-way left turn lane provides motorists with a refuge area and removes them form the traffic while waiting to complete their turns. A constructed example showing these changes is displayed in Figure 4.

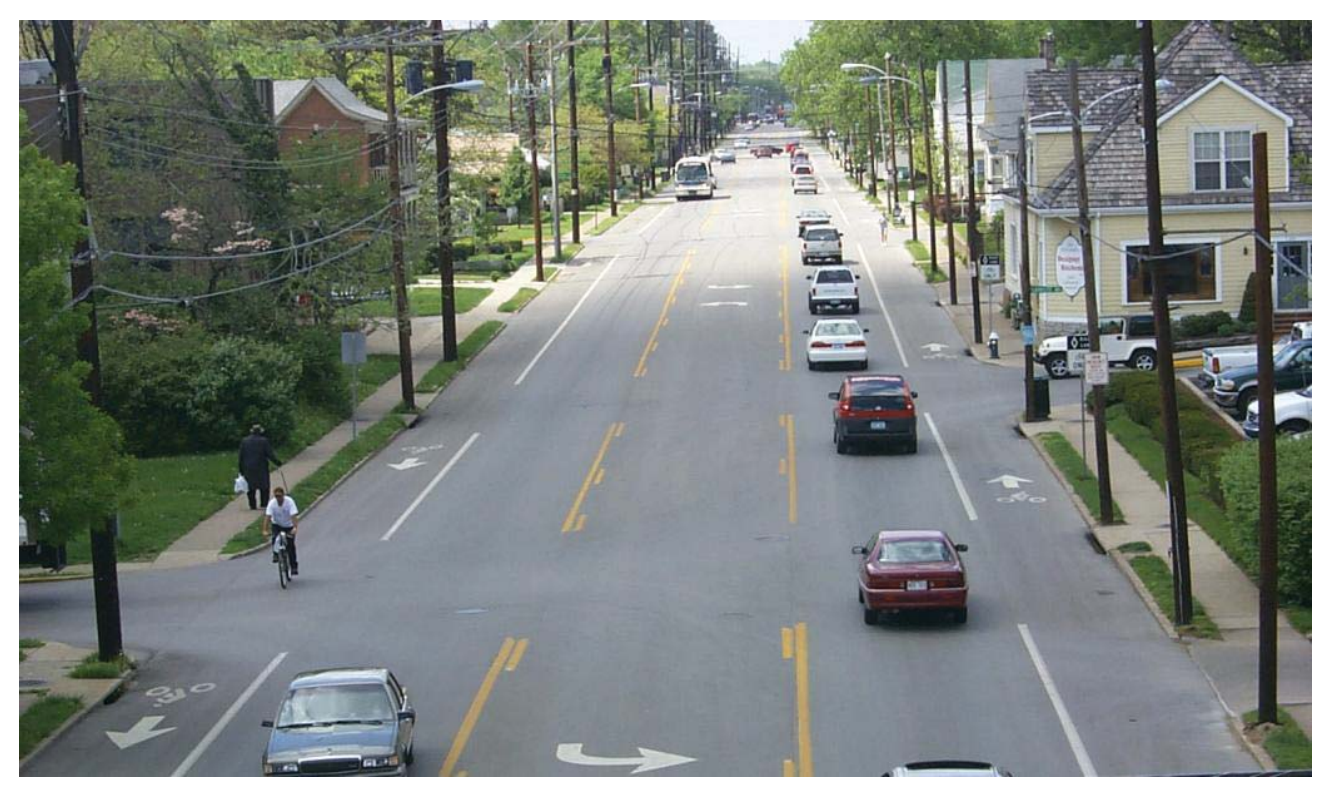

Figure 4: Euclid Post-Road Diet Transformation 


\section{RESULTING CONDITIONS}

This section contains the results concerning travel times and crash history.

\section{Travel Times}

Travel times were collected along the corridor to determine average travel times as well as average speeds for peak travel. Travel times for each direction are shown in Table 1. The travel times were calculated based on 5 runs in each direction.

Table 1: Travel Time Study

\begin{tabular}{ccc}
\hline & \multicolumn{2}{c}{ Travel Time (Seconds) } \\
\cline { 2 - 3 } Run & Northwest & Southeast \\
\hline 1 & 256 & 366 \\
2 & 350 & 342 \\
3 & 372 & 202 \\
4 & 329 & 290 \\
5 & 358 & 348 \\
\hline Average & 333 & 310 \\
\hline
\end{tabular}

The average northwest travel time was 333 seconds (or 5.55 minutes) and the average southeast travel time was 310 seconds (or 5.16 minutes). This means the combined average is 321 seconds (or 5.36 minutes), thus, the average directional trip speed is 12.3 miles per hour. It should be noted that the existing speed limit is 35 miles per hour.

\section{Crash History}

A three-year crash history was collected and analyzed along the corridor. The period evaluated was between January 1, 1997 and December 31, 1999 and there were 236 crashes recorded in this period. Table 2 summarizes the crashes by type and location. Crashes are disaggregated by signalized and midblock crashes to assist in identifying crash patterns that may be affected by the implementation of the road diet. Table 3 summarizes crashes for the three years following the road diet change and the data is similarly arranged as in Table 2. The dates of the crashes evaluated extend from January 1, 2001 to December 31, 2003 and there were 104 crashes during that period. 
Table 2: Pre- Road Diet Three-Year Crash Summary (1997-1999)

\begin{tabular}{lccc}
\hline \multicolumn{1}{c}{ Crash Type } & Total & Signalized & Midblock \\
\hline Head On & 2 & 1 & 1 \\
Backing & 0 & 0 & 0 \\
Opposing left turn & 0 & 0 & 0 \\
Rear end & 170 & 115 & 55 \\
Sideswipe-Opposite direction & 1 & 0 & 1 \\
Sideswipe- Same direction & 9 & 1 & 8 \\
Single vehicle & 2 & 0 & 2 \\
Pedestrian-Bicyclist & 21 & 17 & 4 \\
Other & 31 & 6 & 25 \\
\hline \multicolumn{1}{c}{$\quad$ Total } & $\mathbf{2 3 6}$ & $\mathbf{1 4 0}$ & $\mathbf{9 6}$ \\
\hline
\end{tabular}

Table 3: Post- Road Diet Three-Year Crash Summary (2001-2003)

\begin{tabular}{lccc}
\hline \multicolumn{1}{c}{ Crash Type } & Total & Signalized & Midblock \\
\hline Head On & 3 & 0 & 3 \\
Backing & 1 & 0 & 1 \\
Opposing left turn & 19 & 19 & 0 \\
Rear end & 36 & 9 & 27 \\
Sideswipe-Opposite direction & 3 & 2 & 1 \\
Sideswipe- Same direction & 4 & 1 & 3 \\
Single vehicle & 1 & 0 & 1 \\
Pedestrian-Bicyclist & 2 & 1 & 1 \\
Other & 35 & 17 & 18 \\
\hline \multicolumn{1}{c}{$\quad$ Total } & $\mathbf{1 0 4}$ & $\mathbf{4 9}$ & $\mathbf{5 5}$ \\
\hline
\end{tabular}

The primary crash type at both intersection and midblock locations, and both before and after the road-diet transformation, is rear ends. This is mainly attributed to vehicles slowing to turn left or right. Though this is still the highest type of crash after the road diet is implemented, one can see a significant reduction in the number of crashes. The data shows a $56 \%$ decrease in total crashes (less than half after the road diet) and an accompanying $79 \%$ decrease in rear ends and $30 \%$ decrease in sideswipes. 


\section{EVALUATION METHODOLOGY}

Safety and operational improvements are typically the reasons for a road diet implementation. Therefore, the evaluation should consider metrics that could allow for comparisons of the before and after conditions as well determine the level of performance for the current conditions. These measures include crash changes and travel times and speeds.

\section{Safety Evaluation}

The before and after the road diet comparison of traffic crashes shows a decrease by $56 \%$ in the after period accompanied by a $79 \%$ decrease in rear end crashes and $30 \%$ decrease in sideswipes. It was also found that the percent of crashes with injuries has stayed about the same, but since overall crashes have decrease so have total injuries. These data indicate that the safety of the corridor has improved with the road diet conversion.

It should also be noted that ADT on this section did not increase. In 1996 the ADT was 15,900 vehicles per day and, in 2004 after the road diet, the ADT was 13,700 vehicles per day. This shows that volume has remained virtually the same. The reduction in the ADT does not affect the crash comparison, since the crash rates were also reduced over the same period by $49 \%$ (average crash rate of 1374 crashes/100 million vehiclemiles traveled per year in before period and 703 crashes/100 million vehicle-miles traveled per year in the after period).

Another important aspect is the changes in the crashes for pedestrians and bicyclists. These crashes decreased from 21 to 2 , showing a decrease of $90 \%$. This greatly encourages more bicyclists and pedestrians while making the area safer for them as well as for motorists.

\section{Operations Evaluation}

There is no travel time data available for the before period to allow for a before-after comparison. The current speeds are below the speed limit indicating that there may be some delays during the peak period. The presence of the signalized intersections could induce operational delays which could explain the increased travel time. However, the increased travel times are small and no congestion was observed during the data collection indicating acceptable operation. 
EVALUATION SUMMARY

The analysis shown here helps one to see the benefits and disadvantages of the road diet, allowing them to decide if it was the correct decision. Table 4 presents a summary of the evaluation parameters considered.

Table 4: Summary of Road Diet Installation Effects

\begin{tabular}{|l|l|}
\hline \multicolumn{1}{|c|}{ Aspect } & \multicolumn{1}{|c|}{ Effect } \\
\hline $\begin{array}{l}\text { Travel times and Intersection } \\
\text { delays }\end{array}$ & Undocumented but could be a problem \\
\hline & $\begin{array}{l}\text { Improved; } \\
\text { reduced conflict points; } \\
\text { reduced crashes, especially sideswipe and rear end; } \\
\text { reduced crashes with injuries; } \\
\text { potential to increase rear end crashes at intersections }\end{array}$ \\
\hline Overall safety & Most likely improved uniformity; traffic calming potential \\
\hline Pravel speeds & Improved \\
\hline Multimodal operations & Improved for bicycles and transit \\
\hline Access management & Improved potential \\
\hline
\end{tabular}

Though travel times and intersection delays remain a problem for the corridor at peak times, there are several improvements, especially in safety, that could outweigh the operational delays. The safety reductions both in total crashes and rear end crashes show that there has been a significant safety improvement along the corridor. This is even more so for pedestrian and bicyclists for which the crashes reduced by $90 \%$. Moreover, the aspects that come along with most road diets such as improved traffic calming, improved access management potential, and improved multimodal operations could also be viewed as additional benefits for this corridor. Therefore, it is deemed appropriate to consider this as a successful conversion. 


\section{ROAD DIET CASE STUDY EVALUATION US 27 CAMPBELL COUNTY, KY \\ (FINAL) REPORT}

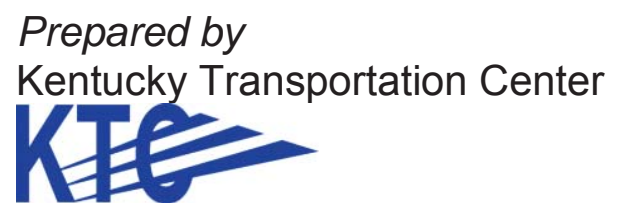

\section{Prepared for:}

Kentucky Transportation Cabinet Division of Planning

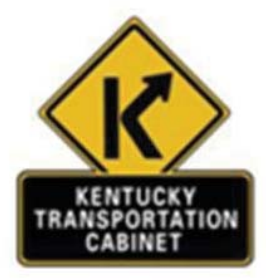

Date:

August 2011 


\section{TABLE OF CONTENTS}

Introduction

\section{LIST OF FIGURES}

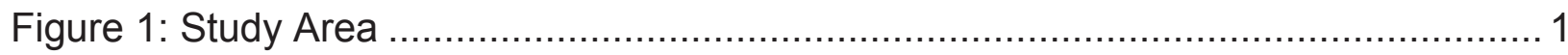

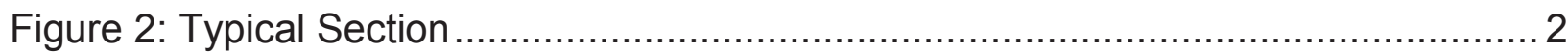

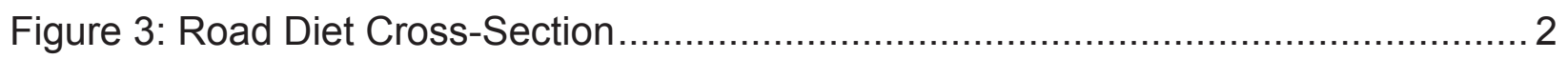

Figure 4: US 27 Post Road Diet Transformation (near ends).................................... 3

Figure 5: US 27 Post Road Diet Transformation (in middle) ..................................... 3

\section{LIST OF TABLES}

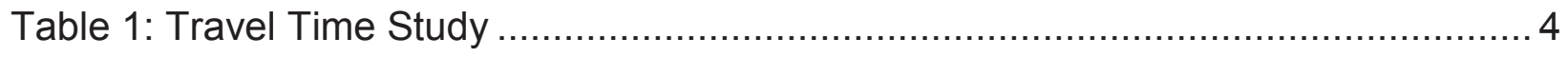

Table 2: Three-Year Crash Summary (2005-2007) ….......................................... 5

Table 3: Two-Year Crash Summary (2009-2010) .................................................. 5

Table 4: Summary of Road Diet Effects ............................................................ 7 


\section{INTRODUCTION}

The purpose of this report is to evaluate and present the effects associated with the conversion of a section of US 27, Campbell County KY which occurred in 2008. This section underwent a "road diet" in which the road was reduced from a 4-lane facility to a 3-lane facility. The area considered in this study extends on US 27 from Camp to Ft. Thomas Avenue (MP 16.721 to MP 18.133) for a distance of 1.412 miles. The location of the study area is shown below in Figure 1.

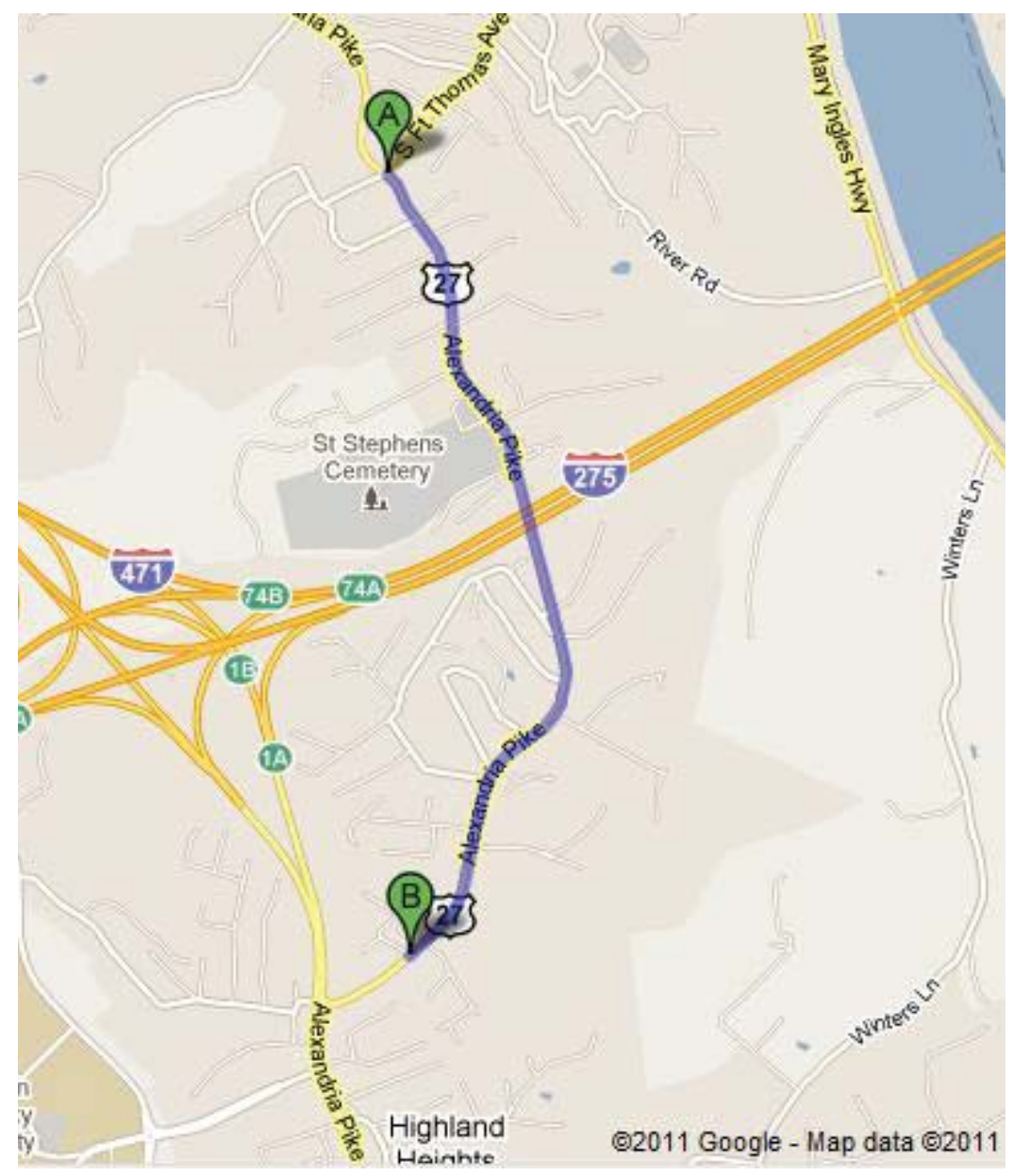

Figure 1: Study Area

This report summarizes the results of the road diet on US 27 while concentrating on aspects such as travel times, crash records, and safety. As well as resulting conditions, former conditions will be discussed. 


\section{ROAD DIET IMPLEMENTATION}

The study section was originally four lanes wide, each about $11 \mathrm{ft}$ in width, with two lanes in each direction. This cross section is shown in Figure 2. It should also be noted that along the 1.412 mile section there are 104 residential or commercial entrances/exits. Furthermore, there are six intersections with traffic signals at Lowes Road, Renshaw Road, Holly Woods Drive, Crowell Avenue, Concord Avenue, and Ft. Thomas Avenue. The frequency of access points indicates that there are several left turns along the corridor. This resulted in frequent backed up traffic in the inside lanes in each direction resulting in the use of these lanes as exclusive left-turn lanes at peak conditions. There is also the potential for unsafe conditions due to the need of through vehicles to avoid the blocked lanes.

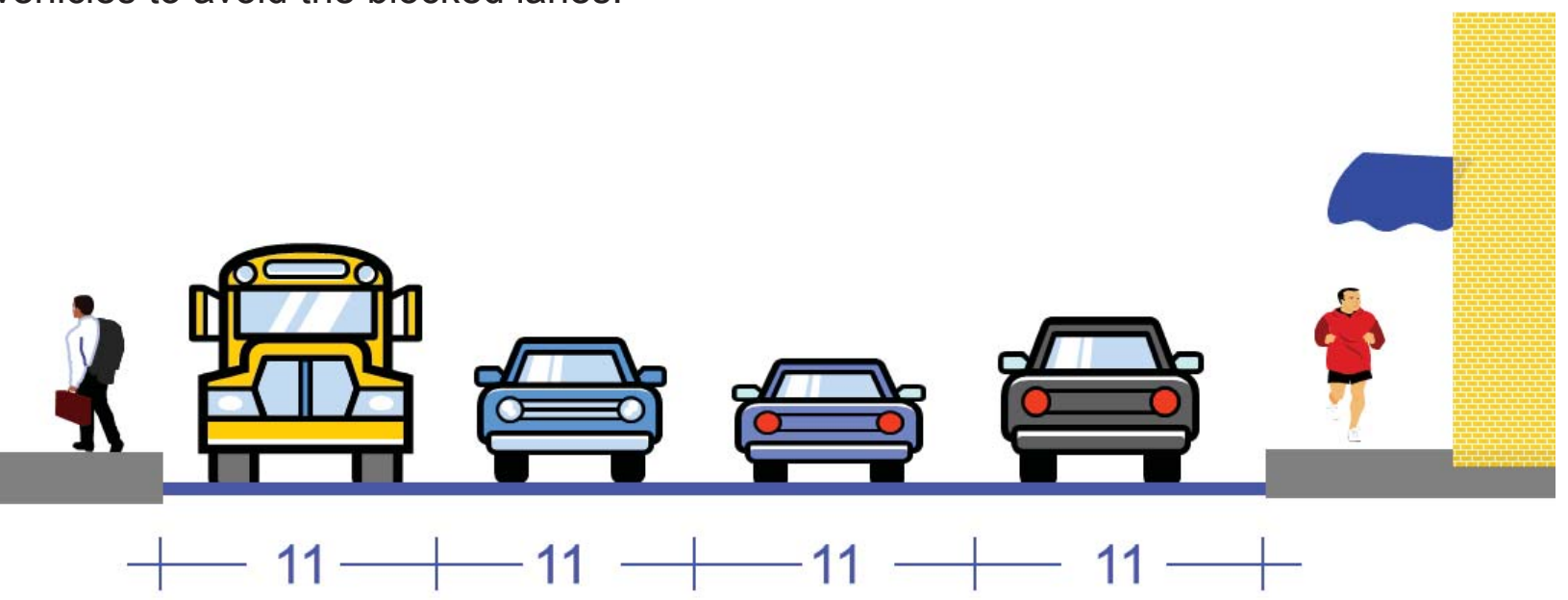

Figure 2: Typical Section

The road diet approach distributed the available roadway width differently to address these issues. This solution took the paved roadway and made it so that only one 11 foot lane remained in each direction while a 12 foot two-way-left-turn lane was added in the center. Additionally, 5-foot bike lanes were added on each side in hopes of increasing multimodal transportation. An example of this cross-section can be seen in Figure3.
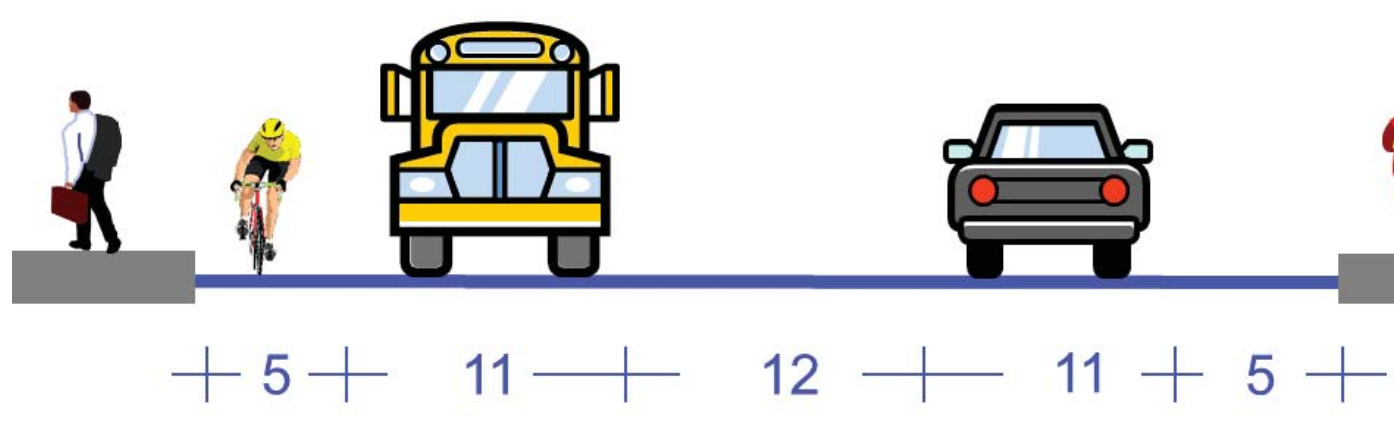

Figure 3: Road Diet Cross-Section 
One of the main goals of the road diet was to improve safety along with improving efficiency. The two-way left turn lane provides motorists with a refuge area and removes them from the traffic while waiting to complete their turns. Constructed examples of the resulting corridor can be seen in Figures $\mathbf{4}$ and $\mathbf{5}$.

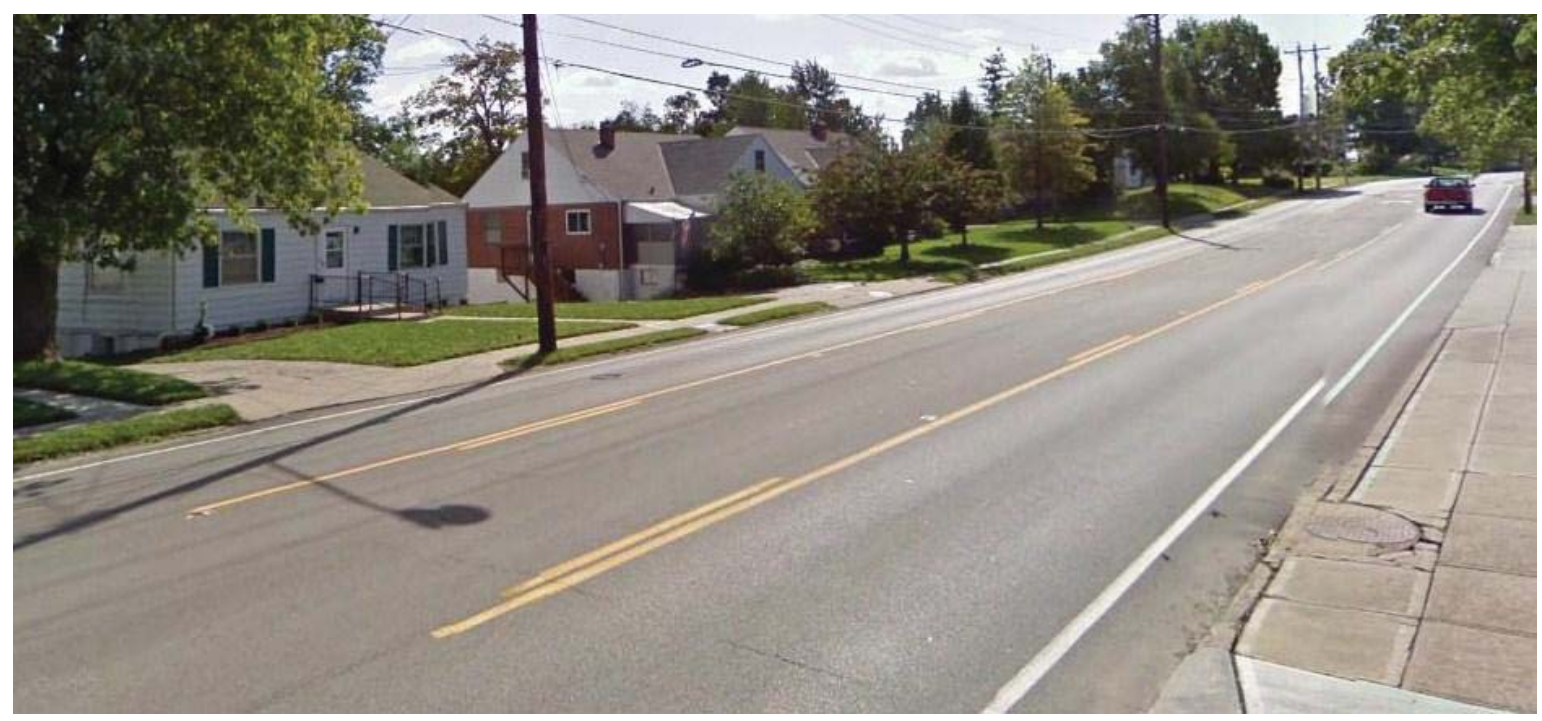

Figure 4: US 27 Post-Road Diet Transformation (Near Ends with More Entrances)

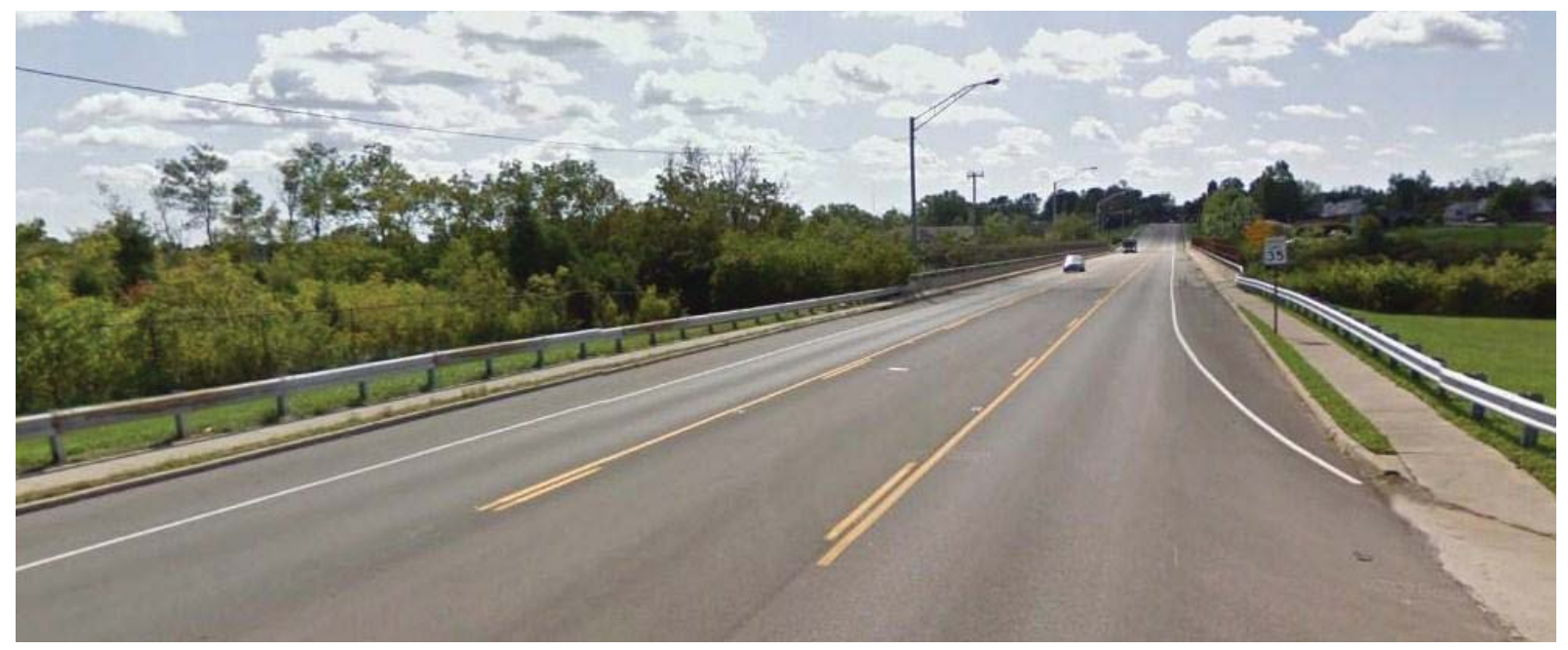

Figure 5: US 27 Post-Road Diet Transformation (In Middle with Few Entrances) 


\section{RESULTING CONDITIONS}

This section contains the results concerning travel times and crash history.

\section{Travel Times}

Travel times were collected along the corridor to determine average travel times as well as average speeds for peak travel. Travel times for each direction are shown in Table 1. The travel times were calculated based on 6 runs in each direction.

Table 1: Travel Time Study

\begin{tabular}{ccc}
\hline & \multicolumn{2}{c}{ Travel Time (Seconds) } \\
\cline { 2 - 3 } Run & North & South \\
\hline 1 & 220 & 190 \\
2 & 210 & 250 \\
3 & 250 & 250 \\
4 & 230 & 320 \\
5 & 310 & 280 \\
6 & 210 & 190 \\
\hline Average & 238 & 247 \\
\hline
\end{tabular}

The average northbound travel time was 238 seconds (or 3.97 minutes) and the average southbound travel time was 247 seconds (or 4.12 minutes). This means the combined average is 242.5 seconds (or 4.04 minutes), thus, the average directional trip speed is 21 miles per hour. It should be noted that the existing speed limit is 35 miles per hour.

\section{Crash History}

A three-year crash history was collected and analyzed along the corridor. The period evaluated was between January 1, 2005 and December 31, 2007 and there were 60 crashes recorded in this period. Table 2 summarizes the crashes by type and location. Crashes are disaggregated by signalized and midblock crashes to assist in identifying crash patterns that may be affected by the implementation of the road diet. Table 3 summarizes crashes for the two years following the road diet change and the data is similarly arranged as in Table 2. The dates of the crashes evaluated extend from January 1, 2009 to December 31, 2010 and there were a total of 19 crashes for this period. 
Table 2: Pre- Road Diet Three-Year Crash Summary (2005-2007)

\begin{tabular}{lccc}
\hline \multicolumn{1}{c}{ Crash Type } & Total & Signalized & Midblock \\
\hline Head On & 1 & 1 & 0 \\
Backing & 5 & 0 & 5 \\
Opposing left turn & 4 & 4 & 0 \\
Rear end & 21 & 4 & 17 \\
Sideswipe-Opposite direction & 2 & 0 & 2 \\
Sideswipe- Same direction & 5 & 1 & 5 \\
Single vehicle & 1 & 1 & 0 \\
Pedestrian-Bicyclist & 0 & 0 & 0 \\
Other & 19 & 2 & 17 \\
\hline \multicolumn{1}{c}{$\quad$ Total } & $\mathbf{6 0}$ & $\mathbf{1 5}$ & $\mathbf{4 7}$ \\
\hline
\end{tabular}

Table 3: Post- Road Diet Three-Year Crash Summary (2009-2010)

\begin{tabular}{lccc}
\hline \multicolumn{1}{c}{ Crash Type } & Total & Signalized & Midblock \\
\hline Head On & 1 & 0 & 1 \\
Backing & 0 & 0 & 0 \\
Opposing left turn & 1 & 1 & 0 \\
Rear end & 8 & 0 & 8 \\
Sideswipe-Opposite direction & 0 & 0 & 0 \\
Sideswipe- Same direction & 2 & 0 & 2 \\
Single vehicle & 1 & 0 & 1 \\
Pedestrian-Bicyclist & 1 & 0 & 1 \\
Other & 5 & 0 & 5 \\
\hline \multicolumn{1}{c}{$\quad$ Total } & $\mathbf{1 9}$ & $\mathbf{1}$ & $\mathbf{1 8}$ \\
\hline
\end{tabular}

The primary crash type at both intersection and midblock locations, and both before and after the road-diet transformation, is rear ends. This is mainly attributed to vehicles slowing to turn left or right. Average crashes per year are used for the comparison, since the two time periods were not the same. These averages show a significant reduction in crashes with a $58 \%$ decrease in total crashes, $43 \%$ decrease in rear ends and $57 \%$ decrease in sideswipes. 


\section{EVALUATION METHODOLOGY}

Safety and operational improvements are typically the reasons for a road diet implementation. Therefore, the evaluation should consider metrics that could allow for comparisons of the before and after conditions as well determine the level of performance for the current conditions. These measures include crash changes and travel times and speeds.

\section{Safety Evaluation}

The comparison of the before and after the road diet periods showed that traffic crashes and rear ends alike decreased greatly. With total crashes decreasing $58 \%$, rear ends decreasing $43 \%$ and sideswipes deceasing $57 \%$, it could easily be concluded that the road diet has increased vehicular safety. At the same time there was a reduction in the ADT from 10,600 in 2004 to 7,410 in 2010 . The reduction in the ADT does not affect the crash comparison, since the crash rates were also reduced over the same period by $32 \%$ (average crash rate of 524 crashes/100 million vehicle-miles traveled per year in before period and 356 crashes/100 million vehicle-miles traveled per year in the after period).

The crash data shows that there were no bicycle crashes before the road diet implementation and one after. It could be hypothesized that this is due to increased numbers of bicyclists along the corridor but this cannot be substantiated, since there are no available bicycle counts along the corridor.

\section{Operations Evaluation}

There is no travel time data available for the before period to allow for a before-after comparison. The current speeds are below the speed limit indicating that there may be some delays during the peak period. The presence of the signalized intersections could induce operational delays which could explain the increased travel time. However, the increased travel times are small and no congestion was observed during the data collection indicating acceptable operation. 


\section{EVALUATION SUMMARY}

The analysis shown here helps one to see the benefits and disadvantages of the road diet, allowing them to decide if it was the correct decision. Table 4 presents a summary of the evaluation parameters considered.

Table 4: Summary of Road Diet Installation Effects

\begin{tabular}{|l|l|}
\hline \multicolumn{1}{|c|}{ Aspect } & \multicolumn{1}{c|}{ Effect } \\
\hline $\begin{array}{l}\text { Travel times and Intersection } \\
\text { delays }\end{array}$ & $\begin{array}{l}\text { Unsure of change, but no congestion so seems } \\
\text { successful in this area }\end{array}$ \\
\hline & $\begin{array}{l}\text { Improved; } \\
\text { reduced conflict points; } \\
\text { reduced crashes and crash rates, especially sideswipe } \\
\text { and rear end; } \\
\text { potential to increase rear end crashes at intersections }\end{array}$ \\
\hline Overall safety & Most likely improved uniformity; traffic calming potential \\
\hline Travel speeds & Unsure \\
\hline Pedestrian safety & Improved for bicycles and transit \\
\hline Multimodal operations & Improved potential \\
\hline Access management &
\end{tabular}

It has been made evident that safety has increased, which in itself is enough reason to support the road diet. However, there are aspects that come along with most road diets such as improved traffic calming, improved access management potential, and improved multimodal operations that cannot be measured. With this said, the value behind this road diet is evident. Also, to users, the value of efficient travel times can be invaluable. Therefore, the absence of visible congestion seems to justify the road diet conversion.

The increased safety and efficient travel times indicate a successful road diet application. The travel time studies conducted during peak hour traffic show the lack of any congestion and indicate efficient operations. For cases similar to this, a road diet conversion seems a very sensible and helpful solution that could be implemented. 


\section{ROAD DIET CASE STUDY EVALUATION KY 1428 FLOYD COUNTY, KY \\ (FINAL) REPORT}

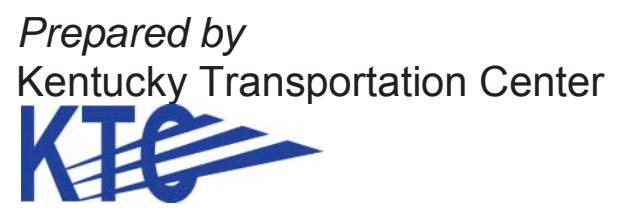

Prepared for:

Kentucky Transportation Cabinet Division of Planning

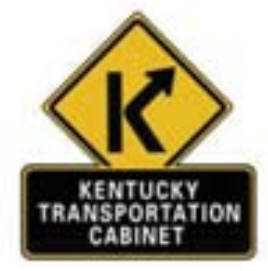

Date:

November 2011 


\section{TABLE OF CONTENTS}

Introduction Road Diet Implementation

\section{LIST OF FIGURES}

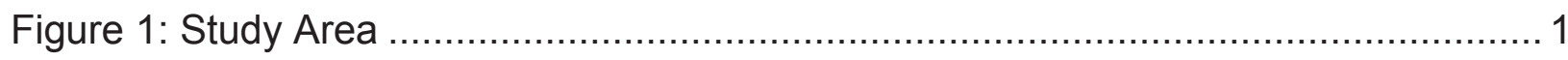

Figure 2: Initial Four Lane Section (North of KY 114) ............................................ 2

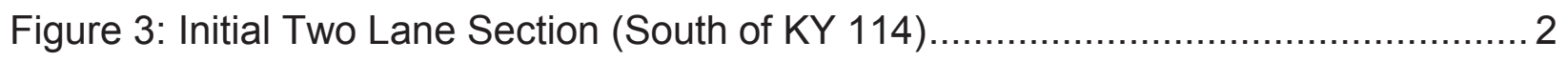

Figure 4: Road Diet Cross Section (North of KY 114) ............................................ 3

Figure 5: Road Diet Cross Section (South of KY 114) .......................................... 3

Figure 6: KY 1428 Post-Road Diet Transformation ............................................... 4

Figure 7: KY 1428 Post-Road Diet Transformation (Transition From Two Lanes) .......... 4

\section{LIST OF TABLES}

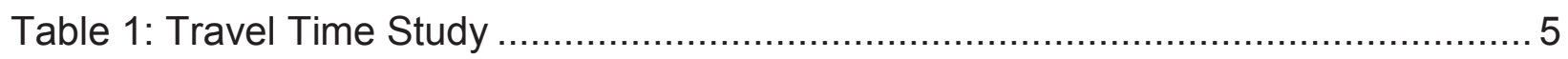

Table 2: Pre- Road Diet Three-Year Crash Summary (2002-2004) .............................. 6

Table 3: Post-Road Diet Three-Year Crash Summary (2007-2009)............................... 6

Table 4: Summary of Road Diet Effects ........................................................... 8 


\section{INTRODUCTION}

The purpose of this report is to evaluate and present the effects associated with the conversion of a section of KY 1428, Floyd County, KY. The project took place between August 2005 and June 2006. This section underwent a "road diet" in which the road was reduced from a 4-lane facility to a 3-lane facility on some parts and grew from a 2-lane facility to a 3-lane facility on the other. The area considered in this study extends on KY 1428 from East Graham Ave to Black Cat Ally (MP 15.153 to MP 16.091) for a distance of 0.928 miles. The location of the study area is shown below in Figure 1.

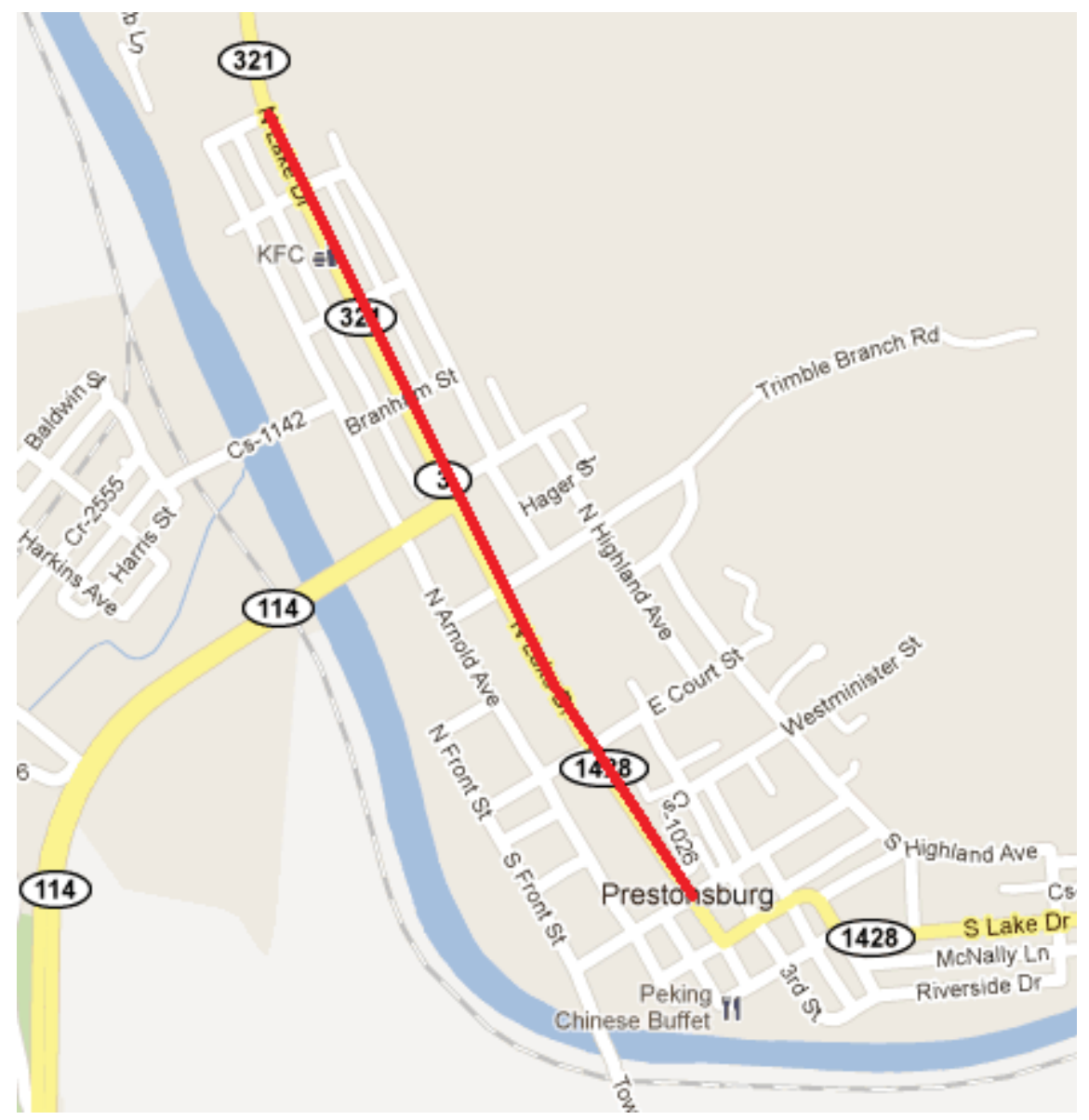

Figure 1: Study Area

This report summarizes the results of the road diet on KY 1428 while concentrating on aspects such as travel times, crash records, and safety. As well as resulting conditions, former conditions will be discussed. 
The study section south of KY 114 was originally four lanes wide, each about 9 feet wide. The section north of KY 114 was two lanes wide, each about 16 feet wide. These cross sections are shown in Figure 2 and Figure 3. It should also be noted that along the 0.928 mile section there are 8 intersections; CS-1026, CR-2556, Friend Street, KY114, Dingus Street, Branham Street, Patton Street, and Music Street. There are also a number of residential and commercial entrances along the study section. The frequency of access points indicates that there are several left turns along the corridor. This resulted in frequent backed up traffic in the inside lanes in each direction resulting in the use of these lanes as exclusive left-turn lanes at peak conditions. There is also the potential for unsafe conditions due to the need of through vehicles to avoid the blocked lanes.

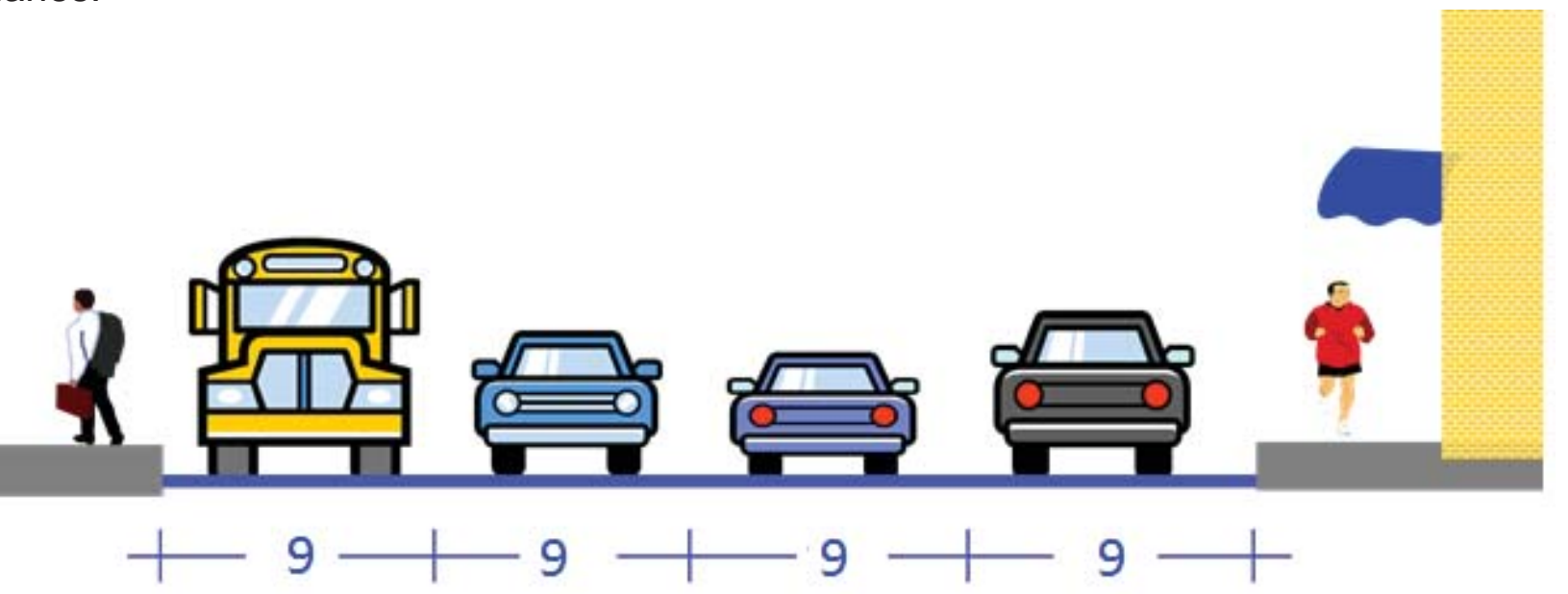

Figure 2: Initial Four Lane Section (North of KY 114)

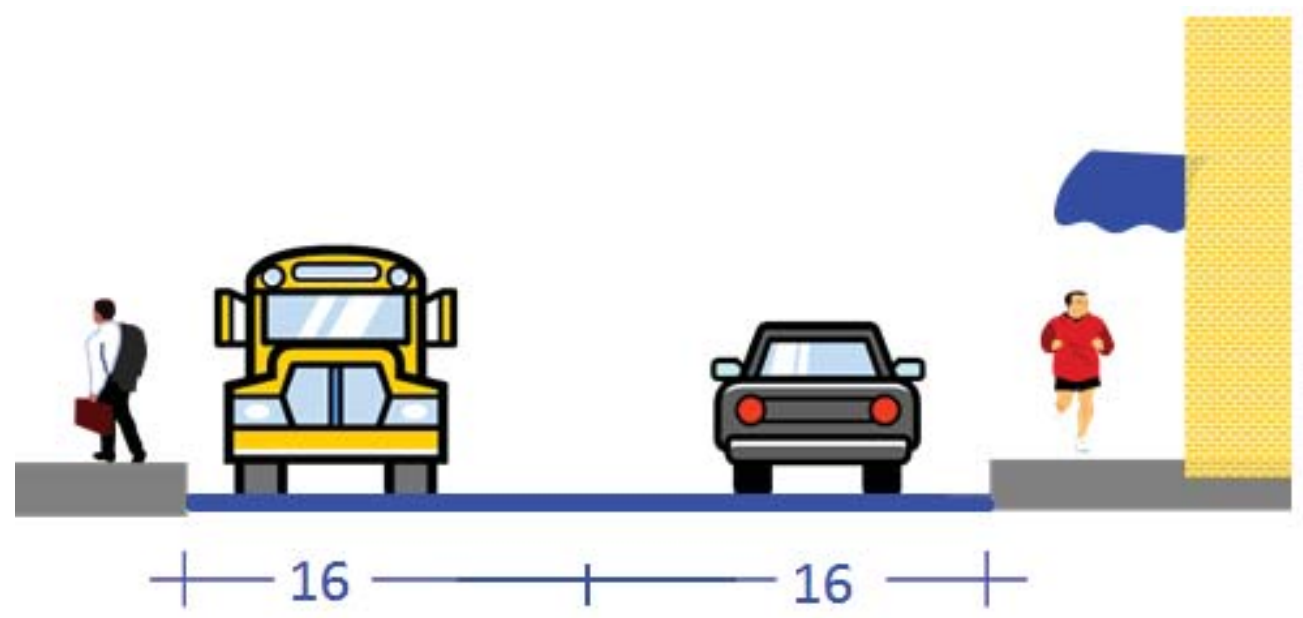

Figure 3: Initial Two Lane Section (South of KY 114) 
The road diet approach distributed the available roadway width differently to address these issues. This solution took the paved roadway on the northern half of the project and converted it into one 12 foot lane in each direction with a 12 foot two-way-left-turn lane in the center. The same thing was done with the southern half of the project utilizing 11 feet lanes instead of 12 feet lanes. An example of these cross-sections can be seen in Figure 4 and Figure 5.

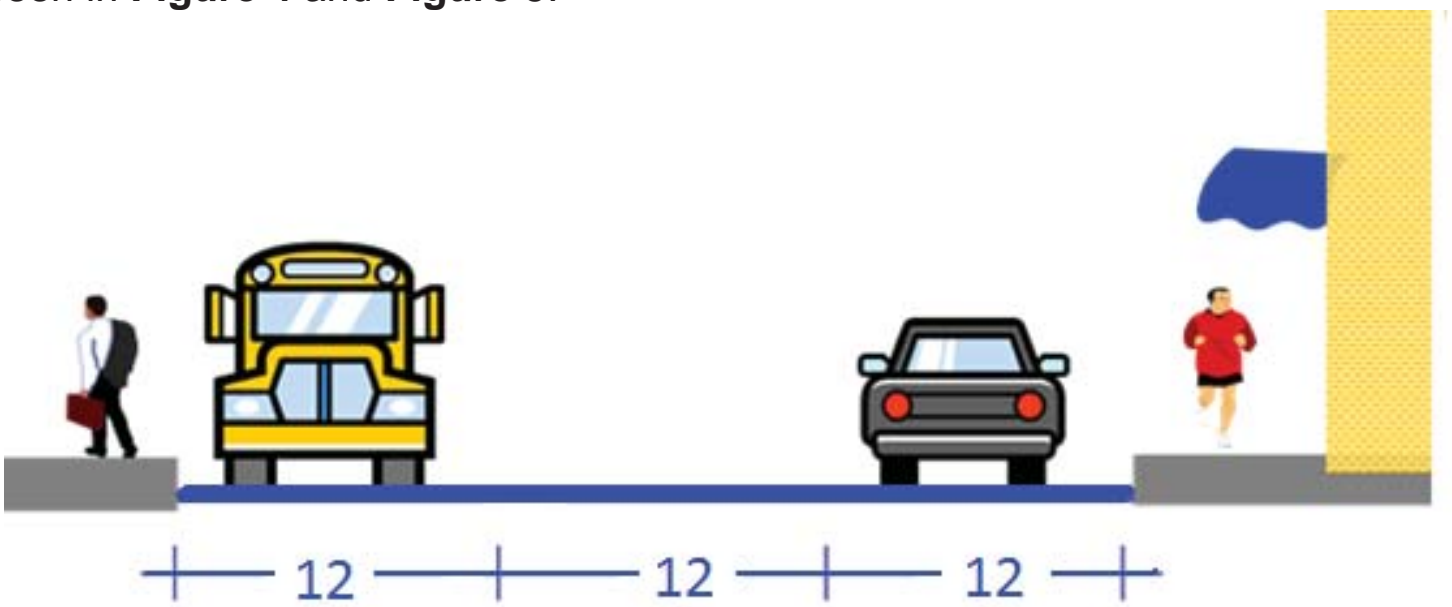

Figure 4: Road Diet Cross-Section (North of 114)

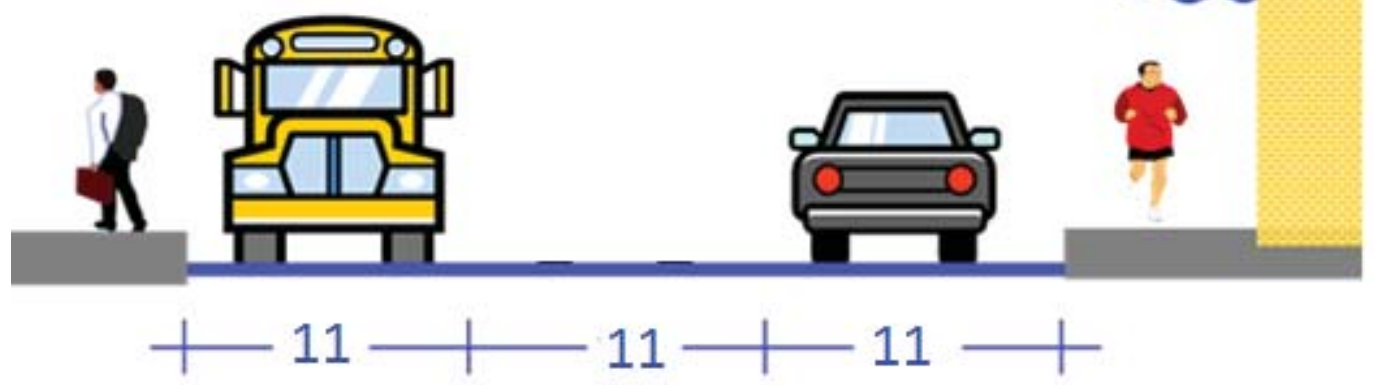

Figure 5: Road Diet Cross-Section (South of 114)

One of the main goals of the road diet was to improve safety along with improving efficiency. The two-way left-turn lane provides motorists with a refuge area and removes them from the traffic while waiting to complete their turns. Constructed examples of the resulting corridor can be seen in Figures 6 and Figure 7. 


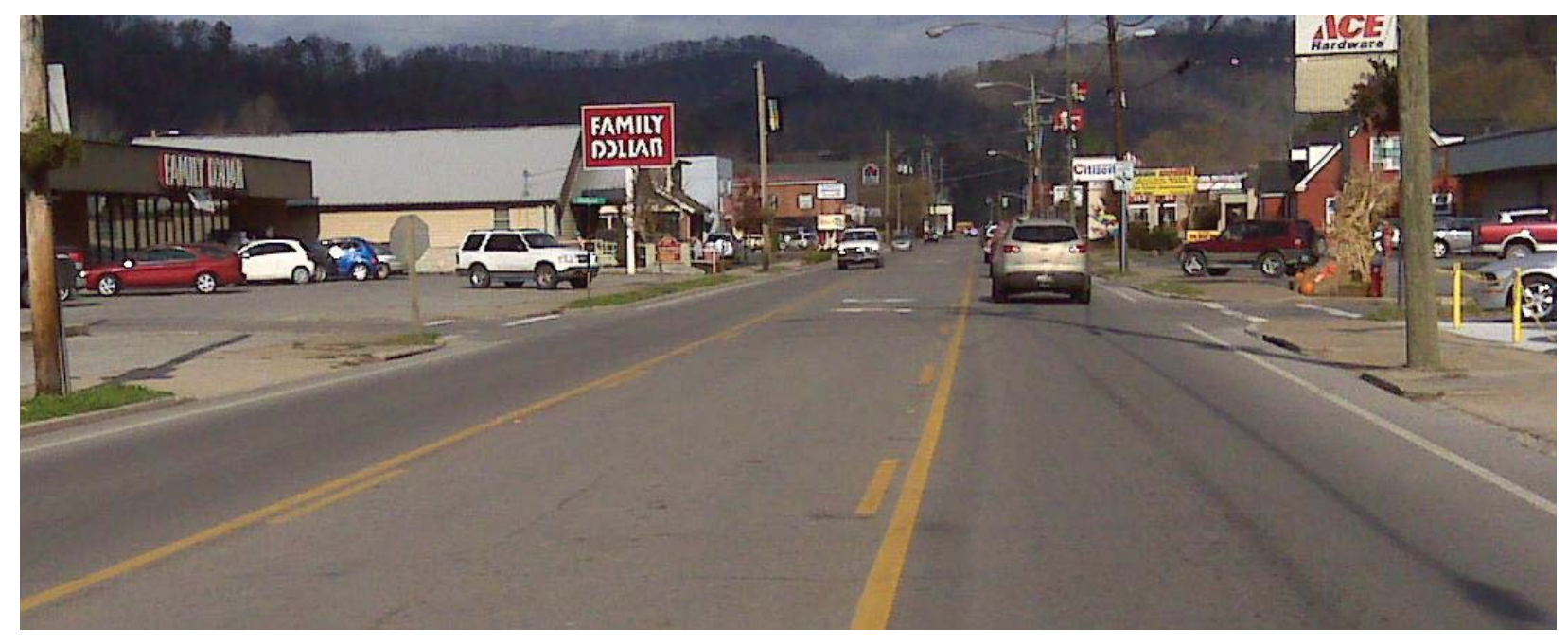

Figure 6: KY 1428 Post-Road Diet Transformation

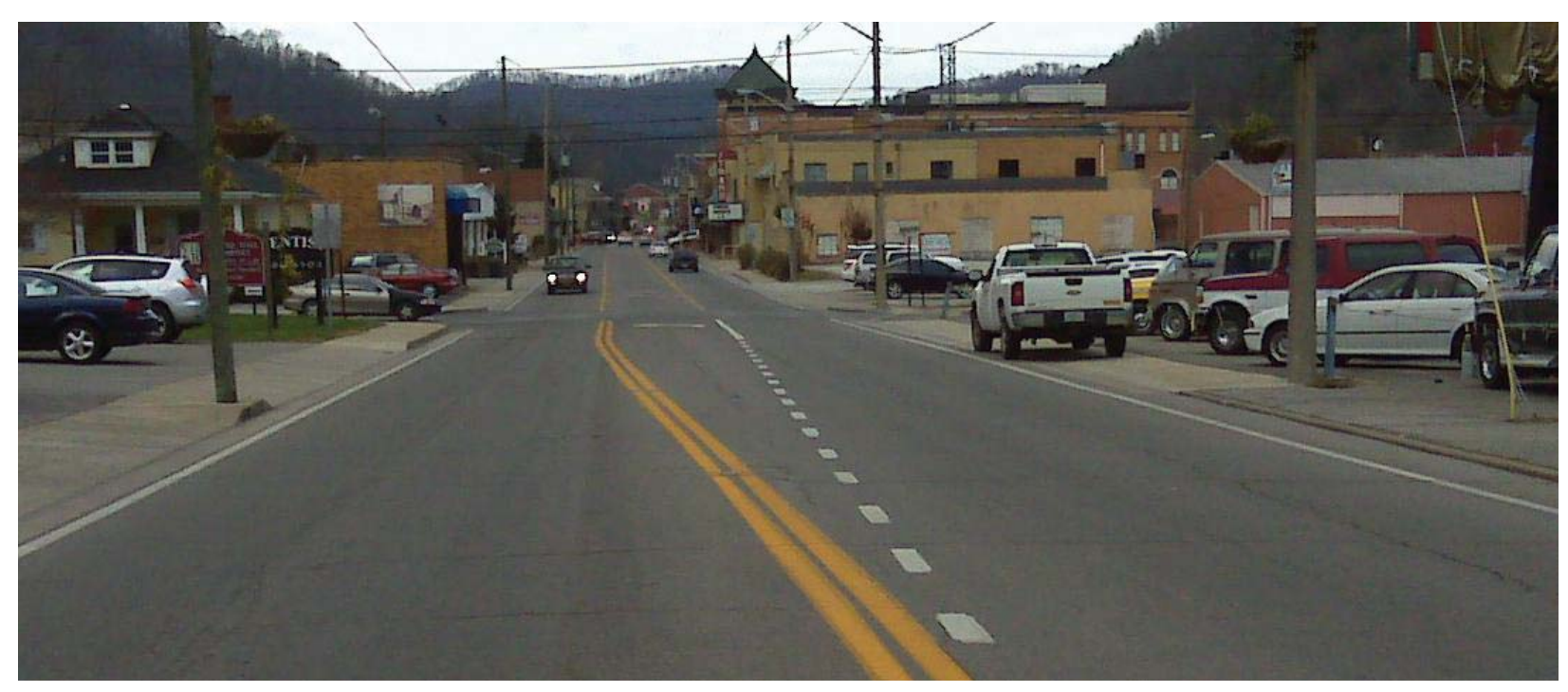

Figure 7: KY 1428 Post-Road Diet Transformation (Transition from Two Lanes) 


\section{RESULTING CONDITIONS}

This section contains the results concerning travel times and crash history.

\section{Travel Times}

Travel times were collected along the corridor to determine average travel times as well as average speeds for peak travel. Travel times for each direction are shown in Table 1.

Table 1: Travel Time Study

\begin{tabular}{cccc}
\hline & & \multicolumn{2}{c}{ Travel Time (Seconds) } \\
\cline { 3 - 4 } Section & Distance(ft) & Northbound & Southbound \\
\hline MP 15.153 to Court Street & 689 & 27 & 18 \\
Court Street to Friend Street & 924 & 29 & 31 \\
Friend Street to KY 114 & 646 & 20 & 17 \\
KY 114 to Music Street & 1745 & 64 & 71 \\
Music Street to MP 16.091 & 823 & 8 & 33 \\
\hline Total & 4827 & 148 & 170 \\
\hline
\end{tabular}

The total northbound travel time was 148 seconds (or 2.47minutes) and the total southbound travel time was 170 seconds (or 2.83 minutes). This means the combined average is 159 seconds (or 2.65 minutes), thus, the average directional trip speed is 21 miles per hour. It should be noted that the existing speed limit is 35 miles per hour.

\section{Crash History}

A three-year crash history was collected and analyzed along the corridor. The period evaluated was between January 1, 2002 and December 31, 2004 and there were 115 crashes recorded in this period. Table 2 summarizes the crashes by type and location. Crashes are disaggregated by signalized and midblock crashes to assist in identifying crash patterns that may be affected by the implementation of the road diet. Table 3 summarizes crashes for the three years following the road diet change and the data is similarly arranged as in Table 2. The dates of the crashes evaluated extend from January 1, 2007 to December 31, 2009 and there were a total of 52 crashes for this period. 
Table 2: Pre- Road Diet Three-Year Crash Summary (2002-2004)

\begin{tabular}{|c|c|c|c|}
\hline Crash Type & Total & Signalized & Midblock \\
\hline Head On & 1 & 0 & 1 \\
\hline Angle & 29 & 1 & 28 \\
\hline Opposing left turn & 5 & 5 & 0 \\
\hline Rear end & 35 & 7 & 28 \\
\hline Sideswipe-Opposite direction & 1 & 0 & 1 \\
\hline Sideswipe- Same direction & 9 & 0 & 9 \\
\hline Single vehicle & 6 & 2 & 4 \\
\hline Pedestrian-Bicyclist & 1 & 0 & 1 \\
\hline Other & 28 & 2 & 26 \\
\hline Total & 115 & 17 & 98 \\
\hline
\end{tabular}

Table 3: Post- Road Diet Three-Year Crash Summary (2007-2009)

\begin{tabular}{lccc}
\hline \multicolumn{1}{c}{ Crash Type } & Total & Signalized & Midblock \\
\hline Head On & 0 & 0 & 0 \\
Angle & 7 & 3 & 4 \\
Opposing left turn & 4 & 4 & 0 \\
Rear end & 29 & 4 & 25 \\
Sideswipe-Opposite direction & 2 & 1 & 1 \\
Sideswipe- Same direction & 4 & 0 & 4 \\
Single vehicle & 1 & 0 & 1 \\
Pedestrian-Bicyclist & 1 & 0 & 1 \\
Other & 4 & 0 & 4 \\
\hline \multicolumn{1}{c}{$\quad$ Total } & $\mathbf{5 2}$ & $\mathbf{1 2}$ & $\mathbf{4 0}$ \\
\hline
\end{tabular}

The primary crash type at both intersection and midblock locations, and both before and after the road-diet transformation, is rear ends. This is mainly attributed to vehicles slowing to turn left or right. The data's yearly averages show a significant reduction in crashes with a $55 \%$ decrease in total crashes, $17 \%$ decrease in rear ends, and $76 \%$ decrease in angle collisions. 


\section{EVALUATION METHODOLOGY}

Safety and operational improvements are typically the reasons for a road diet implementation. Therefore, the evaluation should consider metrics that could allow for comparisons of the before and after conditions as well determine the level of performance for the current conditions. These measures include crash changes and travel times and speeds.

\section{Safety Evaluation}

The comparison of the before and after the road diet periods showed that traffic crashes and angle collisions alike decreased greatly. With total crashes decreasing $55 \%$, rear ends decreasing $17 \%$ and angle collisions decreasing $76 \%$, it could easily be concluded that the road diet has increased vehicular safety. At the same time there was little change in the ADT from 15,939 in 2005 to 16,159 in 2009. The small growth in the ADT show that a decrease in traffic is not the cause of improved safety, since the crash rates were reduced over the same period by 55\% (average crash rate of 618 crashes/100 million vehicle-miles traveled in before period and 281 crashes per 100 million vehiclemiles traveled in the after period). It should also be noted that the number of injury crashes was also reduced from 34 to 14 . This decrease of $59 \%$ is even larger than the decrease in total crashes and suggests that the road diet has impacted severe crashes as well.

The crash data shows that there was one bicycle/pedestrian crash before the road diet implementation and one after. It could be hypothesized that this is due to increased numbers of bicyclists and pedestrians along the corridor but this cannot be substantiated, since there are no available bicycle/pedestrian counts along the corridor.

\section{Operations Evaluation}

There is no travel time data available for the before period to allow for a before-after comparison. The current speeds are below the speed limit indicating that there may be some delays during the peak period. The presence of the signalized intersections could induce operational delays which could explain the increased travel time. However, the increased travel times are small and no congestion was observed during the data collection indicating acceptable operation. 


\section{EVALUATION SUMMARY}

The analysis shown here helps one to see the benefits and disadvantages of the road diet, allowing them to decide if it was the correct decision. Table 4 presents a summary of the evaluation parameters considered.

Table 4: Summary of Road Diet Installation Effects

\begin{tabular}{|l|l|}
\hline \multicolumn{1}{|c|}{ Aspect } & \multicolumn{1}{c|}{ Effect } \\
\hline $\begin{array}{l}\text { Travel times and Intersection } \\
\text { delays }\end{array}$ & $\begin{array}{l}\text { Unsure of change, but no congestion so seems } \\
\text { successful in this area }\end{array}$ \\
\hline & $\begin{array}{l}\text { Improved; } \\
\text { reduced conflict points; } \\
\text { reduced crashes and crash rates, especially angle and } \\
\text { rear end collisions; } \\
\text { potential to increase rear end crashes at intersections }\end{array}$ \\
\hline Overall safety & Most likely improved uniformity; traffic calming potential \\
\hline Travel speeds & Unsure but shoulder gives more space from traffic \\
\hline Pedestrian safety & Unsure \\
\hline Multimodal operations & Improved potential \\
\hline Access management &
\end{tabular}

It has been made evident that safety has increased, which in itself is enough reason to support the road diet. However, there are aspects that come along with most road diets such as improved traffic calming, improved access management potential, and improved multimodal operations that cannot be measured. With this said, the value behind this road diet is evident. Also, to users, the value of efficient travel times can be invaluable. Therefore, the absence of visible congestion seems to justify the road diet conversion.

The increased safety and efficient travel times indicate a successful road diet application. The travel time studies conducted during peak hour traffic show the lack of any congestion and indicate efficient operations. For cases similar to this, a road diet conversion seems a very sensible and helpful solution that could be implemented. 


\section{ROAD DIET CASE STUDY EVALUATION US 127 HARRODSBURG, KY \\ (FINAL) REPORT}

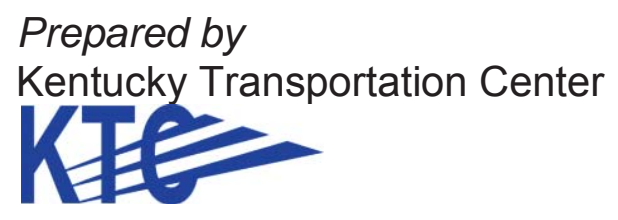

Prepared for:

Kentucky Transportation Cabinet Division of Planning

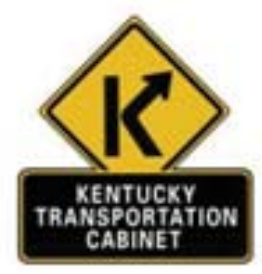

Date:

August 2011 


\section{TABLE OF CONTENTS}

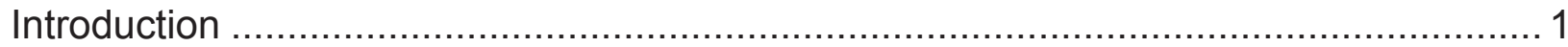

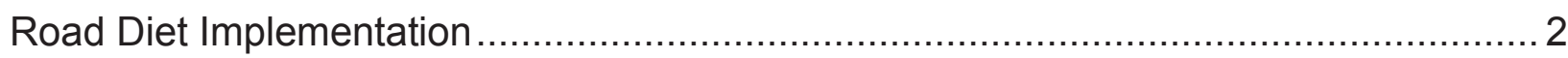

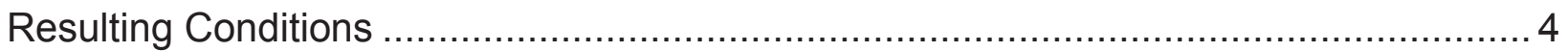

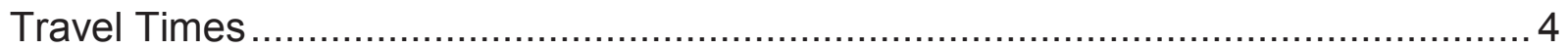

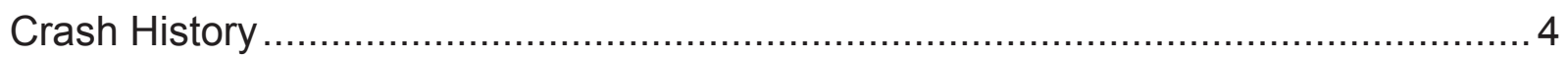

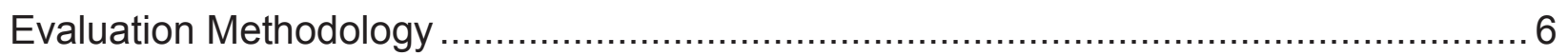

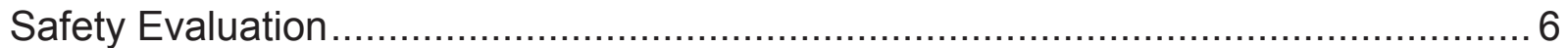

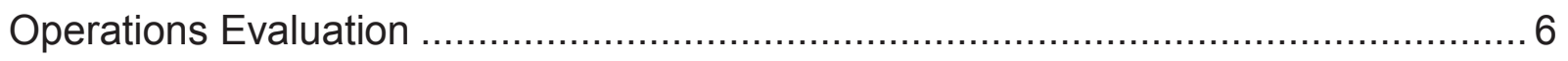

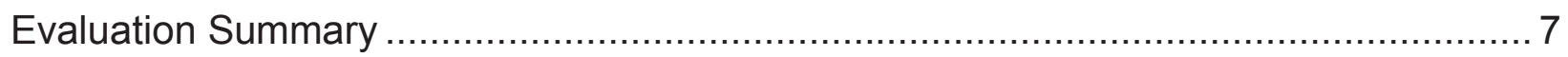

\section{LIST OF FIGURES}

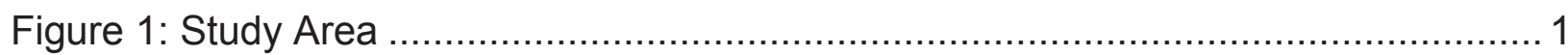

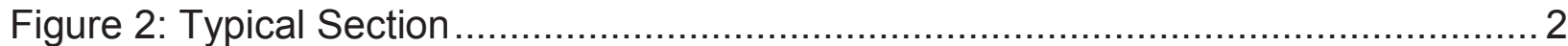

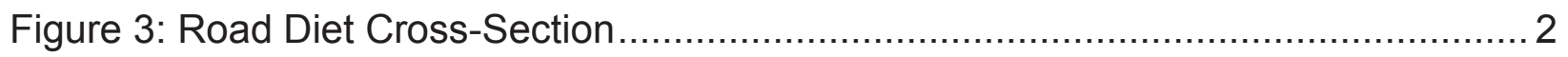

Figure 4: US 127 Post Road Diet Transformation .......................................... 3

\section{LIST OF TABLES}

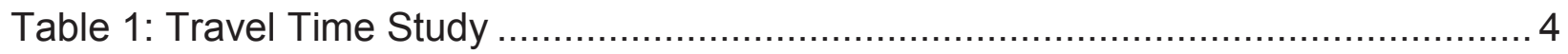

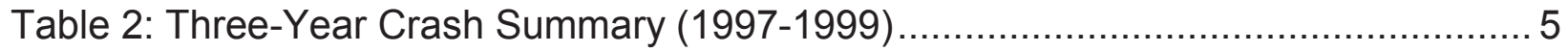

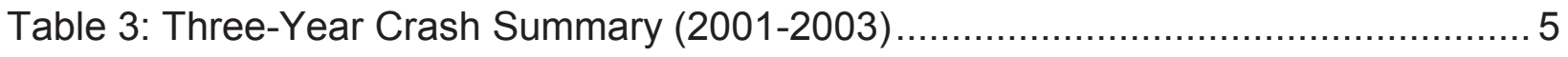

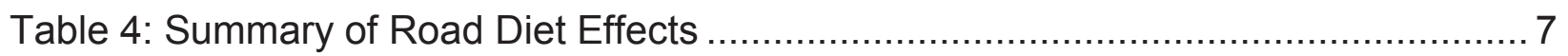




\section{INTRODUCTION}

The purpose of this report is to evaluate and present the effects associated with the conversion of a section of US 127 in Harrodsburg, Mercer County KY. This section underwent a "road diet" in 2006 in which the road was reduced from a 4-lane facility to a 3-lane facility. The area considered in this study extends on US 127 from KY 1989 to Chestnut Street (MP 4.076 to MP 4.893) for a distance of 0.817 miles. The location of the study area is shown below in Figure 1.

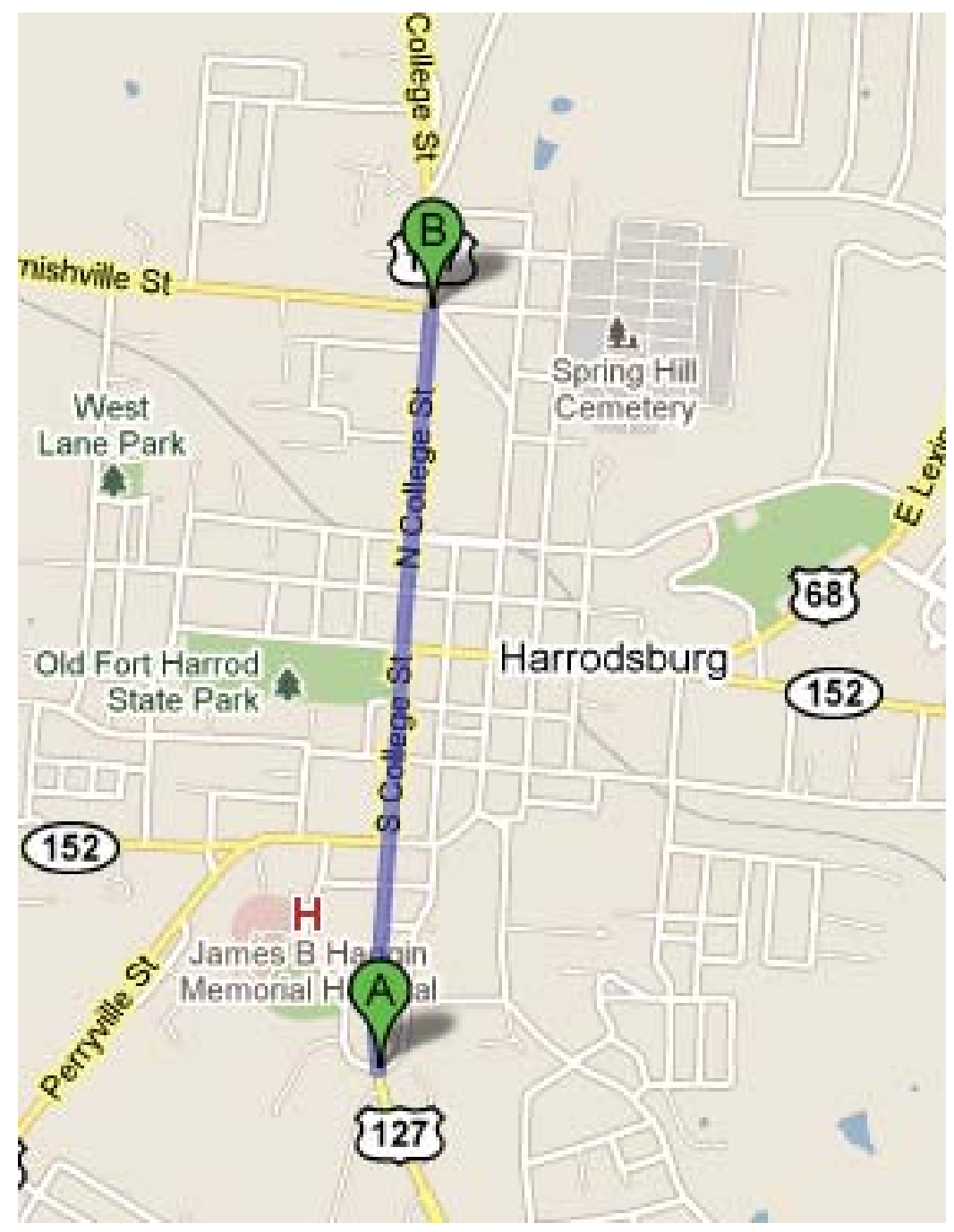

\section{Figure 1: Study Area}

This report summarizes the results of the road diet on College Street while concentrating on aspects such as travel times, crash records, and safety. As well as resulting conditions, former conditions will be discussed. 


\section{ROAD DIET IMPLEMENTATION}

The study section was originally four lanes wide, each about 10 or $11 \mathrm{ft}$ in width, with two lanes in each direction. This cross section is shown in Figure 2. It should also be noted that along the 0.817 mile section there are 117 unsignalized entrances/exits. Furthermore, there are two signalized intersections at Lexington Street and Mooreland Avenue. The frequency of access points indicates that there are several left turns along the corridor. This resulted in frequent backed up traffic in the inside lanes in each direction resulting in the use of these lanes as exclusive left-turn lanes at peak conditions. There is also the potential for unsafe conditions due to the need of through vehicles to avoid the blocked lanes.

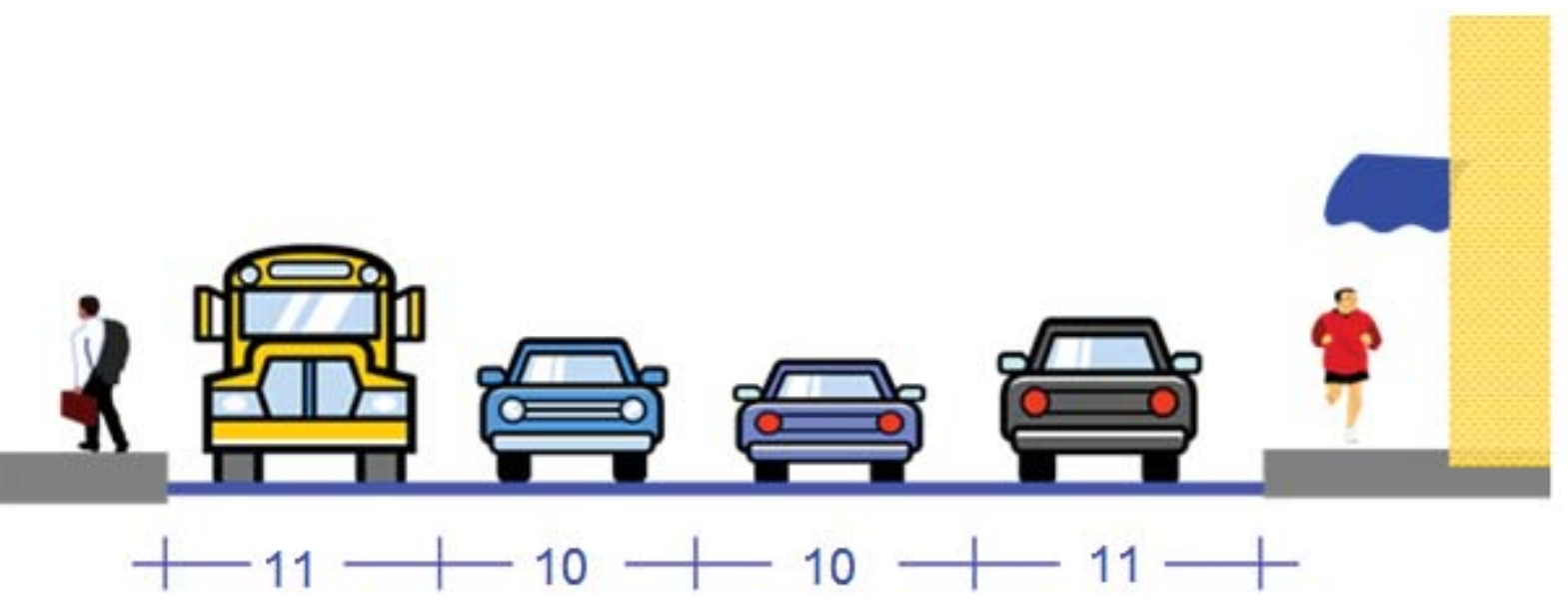

Figure 2: Typical Section

For US 127, rear end crashes were becoming a problem as was congestion. The road diet conversion was implemented to address these issues. The new design distributed the available roadway by providing one 15-foot lane in each direction while adding a 12foot two-way-left-turn lane in the center. The wider travel lanes provide somewhat of a "shoulder" so cars will be farther away from pedestrians. This shoulder also helps make bicyclists traveling on the road feel safer. An example of this cross-section can be seen in Figure3.

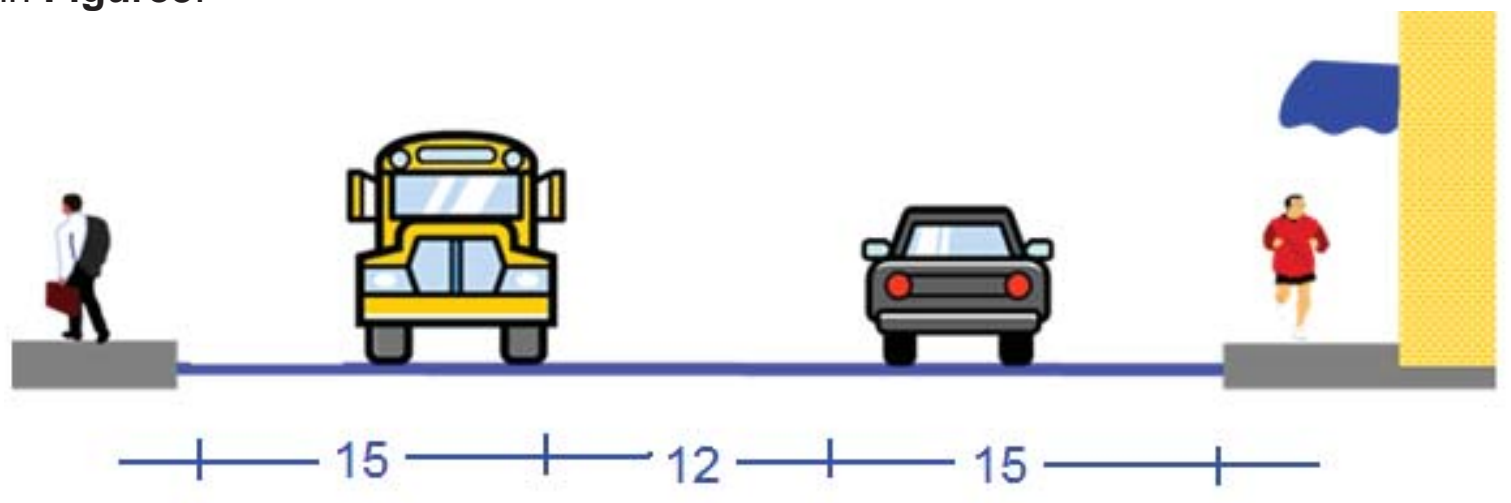

Figure 3: Road Diet Cross-Section 
One of the main goals of the road diet was to improve safety along with improving efficiency. The two-way left turn lane provides motorists with a refuge area and removes them form the traffic while waiting to complete their turns. A constructed example of these changes can be seen in Figure 4.

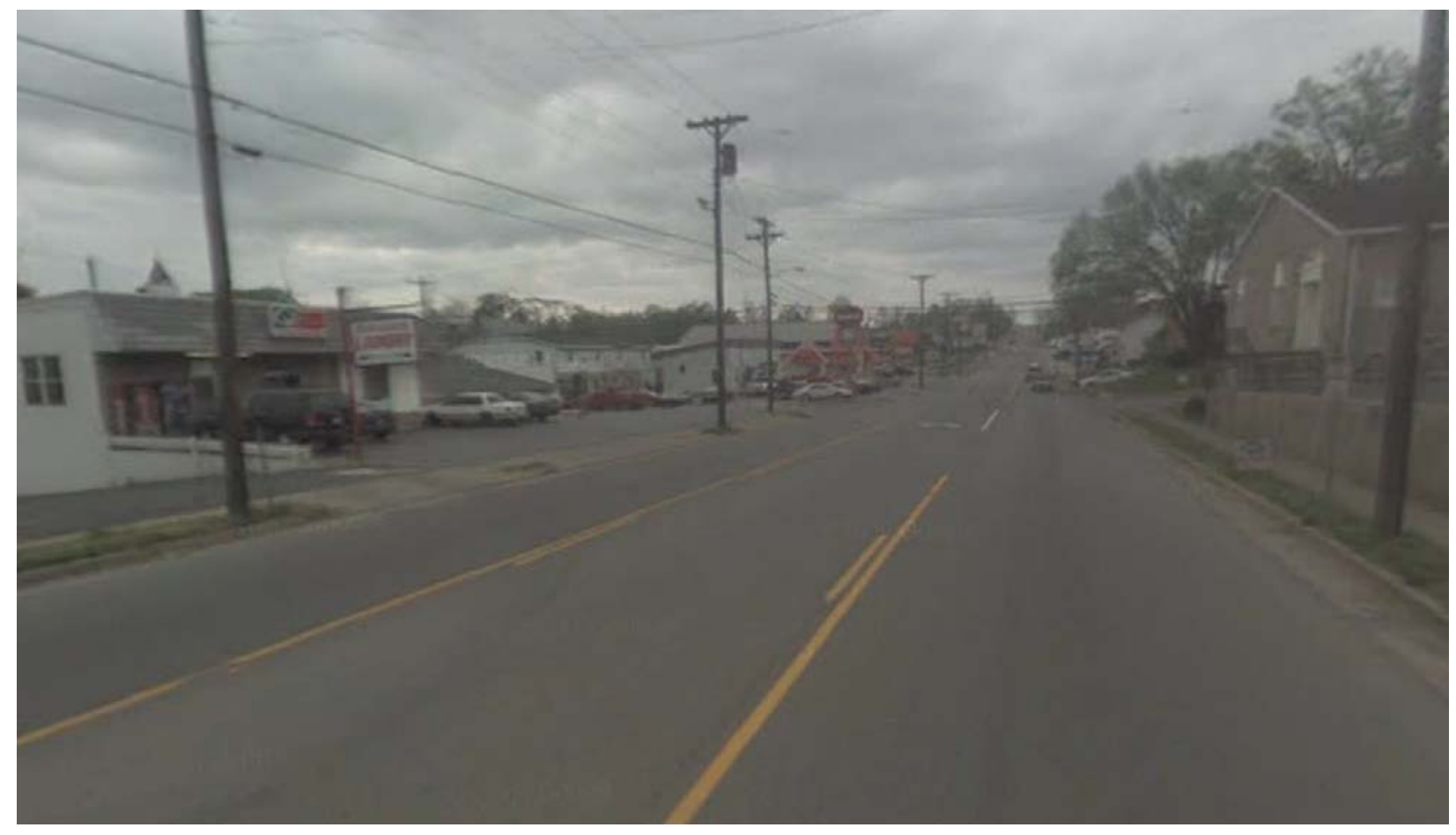

Figure 4: US 127 Post-Road Diet Transformation 


\section{RESULTING CONDITIONS}

This section contains the results concerning travel times and crash history.

\section{Travel Times}

Travel times were collected along the corridor to determine average travel times as well as average speeds for rush hour travel. Travel times for each direction are shown in Table 1. The travel times were calculated based on 5 runs in each direction.

Table 1: Travel Time Study

\begin{tabular}{ccc}
\hline & Travel Time & (Seconds) \\
\cline { 2 - 3 } Run & South & North \\
\hline 1 & 200 & 230 \\
2 & 220 & 260 \\
3 & 240 & 170 \\
4 & 270 & 180 \\
5 & 240 & 200 \\
\hline Average & 234 & 208 \\
\hline
\end{tabular}

The average southbound travel time was 234 seconds (or 3.90 minutes) and the average northbound travel time was 208 seconds (or 3.47 minutes). This means the combined average is 221 seconds (or 3.68 minutes), thus, the average directional trip speed is 13 miles per hour. It should be noted that the existing speed limit is 35 miles per hour.

\section{Crash History}

A three-year crash history was collected and analyzed along the corridor. The period evaluated was between January 1, 2003 and December 31, 2005 and there were 164 crashes recorded in this period. Table 2 summarizes the crashes by type and location. Crashes are disaggregated by signalized and midblock crashes to assist in identifying crash patterns that may be affected by the implementation of the road diet. Table 3 summarizes crashes for the three years following the road diet change and the data is similarly arranged as in Table 2. The dates of the crashes evaluated extend from January 1, 2007 to December 31, 2009 with a total of 97 crashes for the period. 
Table 2: Pre- Road Diet Three-Year Crash Summary (2003-2005)

\begin{tabular}{lccc}
\hline \multicolumn{1}{c}{ Crash Type } & Total & Signalized & Midblock \\
\hline Head On & 5 & 5 & 0 \\
Backing & 2 & 1 & 1 \\
Opposing left turn & 21 & 20 & 1 \\
Rear end & 60 & 20 & 40 \\
Sideswipe-Opposite direction & 4 & 0 & 4 \\
Sideswipe- Same direction & 13 & 7 & 6 \\
Single vehicle & 3 & 2 & 1 \\
Pedestrian-Bicyclist & 0 & 0 & 0 \\
Other & 56 & 34 & 22 \\
\hline \multicolumn{1}{c}{$\quad$ Total } & $\mathbf{1 6 4}$ & $\mathbf{8 9}$ & $\mathbf{7 5}$ \\
\hline
\end{tabular}

Table 3: Post- Road Diet Three-Year Crash Summary (2007-2009)

\begin{tabular}{|c|c|c|c|}
\hline Crash Type & Total & Signalized & Midblock \\
\hline Head On & 0 & 0 & 0 \\
\hline Backing & 5 & 1 & 4 \\
\hline Opposing left turn & 13 & 13 & 0 \\
\hline Rear end & 46 & 9 & 37 \\
\hline Sideswipe-Opposite direction & 0 & 0 & 0 \\
\hline Sideswipe- Same direction & 4 & 2 & 2 \\
\hline Single vehicle & 2 & 1 & 1 \\
\hline Pedestrian-Bicyclist & 0 & 0 & 0 \\
\hline Other & 27 & 10 & 17 \\
\hline Total & 97 & 36 & 61 \\
\hline
\end{tabular}

The primary crash type at both intersection and midblock locations, and both before and after the road-diet transformation, is rear ends. This is mainly attributed to vehicles slowing to turn left or right. Though this is still the highest type of crash after the road diet is implemented, a significant reduction in the number of crashes is noted. The data shows that there a $41 \%$ decrease in total crashes, a $23 \%$ reduction in rear ends and a $76 \%$ reduction in sideswipes. 


\section{EVALUATION METHODOLOGY}

Safety and operational improvements are typically the reasons for a road diet implementation. Therefore, the evaluation should consider metrics that could allow for comparisons of the before and after conditions as well determine the level of performance for the current conditions. These measures include crash changes and travel times and speeds.

\section{Safety Evaluation}

The comparison of the before and after the road diet periods showed that traffic crashes decrease by $41 \%$ and the leading cause of these crashes, rear ends, decreased by $23 \%$. Also, sideswipe crashes were reduced by $76 \%$. The injury crashes also reduced from 44 in the before period to 10 after indicating a significant decrease $(77 \%)$. The data shows that a significant safety improvement along US 127 occurred due to the road diet conversion.

It should also be noted that ADT on this section did not increase. In 2002 the ADT was 12,600 vehicles per day and in 2009 after the road diet, the ADT was 11,300 vehicles per day. This shows that volume has remained virtually unchanged. The reduction in the ADT does not affect the crash comparison, since the crash rates were also reduced over the same period by $34 \%$ (average crash rate of 1205 crashes/100 million vehiclemiles traveled per year in before period and 795 crashes/100 million vehicle-miles traveled per year in the after period).

The data in Tables $\mathbf{2}$ and $\mathbf{3}$ indicate that there were no pedestrian and bicycle crashes before or after the road diet implementation. This could either indicate the absence of any problems or the absence of these users. The lack of any pedestrian and bicycle traffic counts does not allow for any additional conclusions.

\section{Operations Evaluation}

There is no travel time data available for the before period to allow for a before-after comparison. The current speeds are below the speed limit indicating that there may be some delays during the peak period. The presence of the signalized intersections could induce operational delays which could explain the increased travel time. However, the increased travel times are small and no congestion was observed during the data collection indicating acceptable operation. 


\section{EVALUATION SUMMARY}

The analysis shown here helps one to see the benefits and disadvantages of the road diet, helping decide if it was the correct decision. Table 4 presents a summary of the evaluation parameters considered.

Table 4: Summary of Road Diet Installation Effects

\begin{tabular}{|l|l|}
\hline \multicolumn{1}{|c|}{ Aspect } & \multicolumn{1}{c|}{ Effect } \\
\hline $\begin{array}{l}\text { Travel times and Intersection } \\
\text { delays }\end{array}$ & Seems to run smooth. \\
\hline Overall safety & $\begin{array}{l}\text { Improved; } \\
\text { reduced conflict points; } \\
\text { reduced crashes, especially sideswipe; } \\
\text { reduced crashes with injuries; } \\
\text { potential to increase rear end crashes at intersections }\end{array}$ \\
\hline Travel speeds & Most likely improved uniformity; traffic calming potential \\
\hline Pedestrian safety & Unchanged \\
\hline Multimodal operations & Unchanged but encourage bicyclists more \\
\hline Access management & Improved potential \\
\hline
\end{tabular}

The before and after crash data indicate a significant safety improvement along this corridor supporting the road diet conversion. In addition, the intangible benefits of road diets such as improved traffic calming, improved access management potential, and improved multimodal operations, could further support the benefits of the conversion. Though an increase in bicyclists is not evident, at least the road diet (with its wider lanes) encourages more bicyclists to use the roads. With this said, the value behind this road diet is evident.

The travel time studies show that the road diet has helped traffic move smoothly as well as safely. The safety improvements are significant and the road diet conversion has contributed to this and thus it is would be recommended for cases similar to this for implementation. 


\section{ROAD DIET CASE STUDY EVALUATION \\ KY 74 MIDDLESBORO, KY \\ KYTC DISTRICT 11 \\ FINAL REPORT}

\section{Prepared by \\ Kentucky Transportation Center

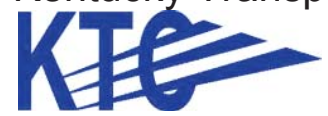

Prepared for:

Kentucky Transportation Cabinet

Division of Planning

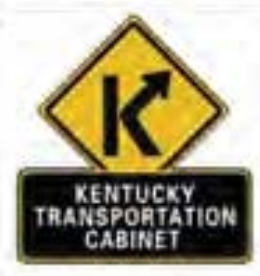

Date:

December 2010 


\section{TABLE OF CONTENTS}

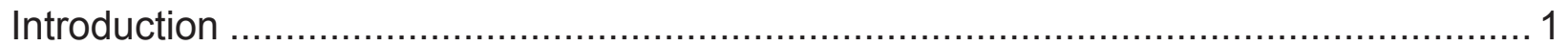

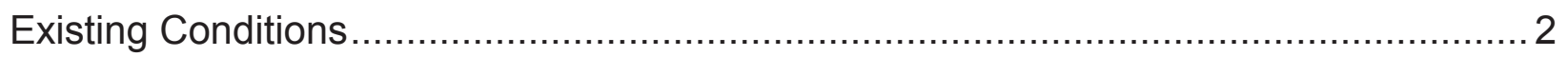

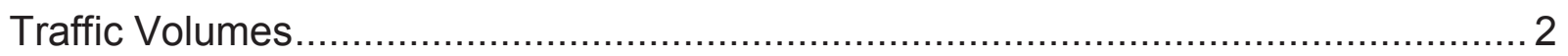

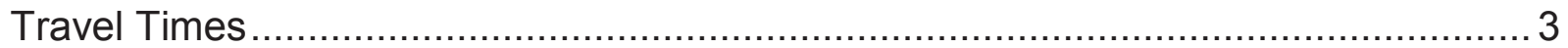

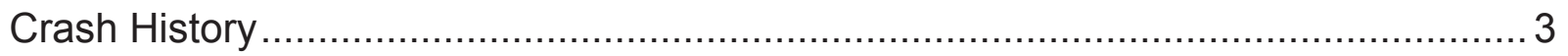

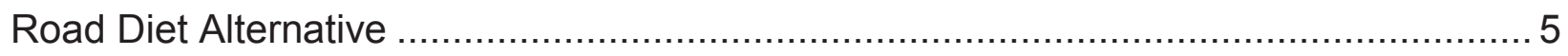

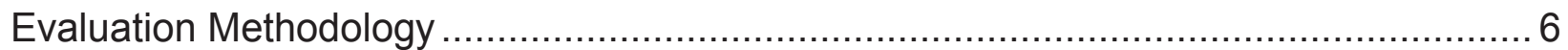

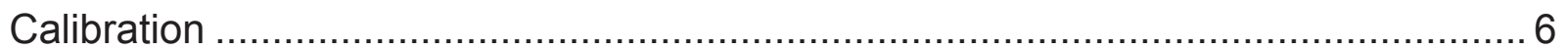

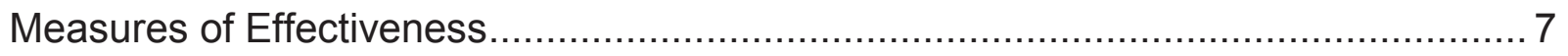

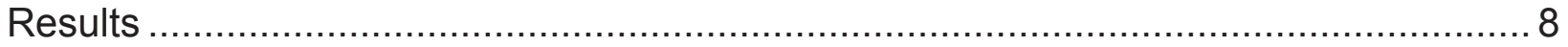

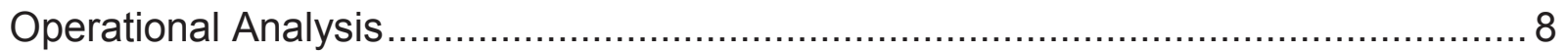

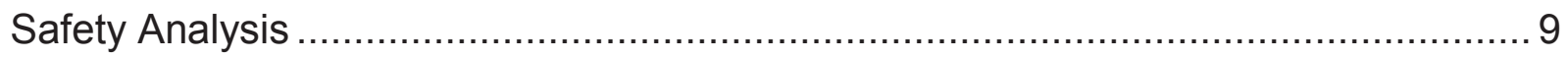

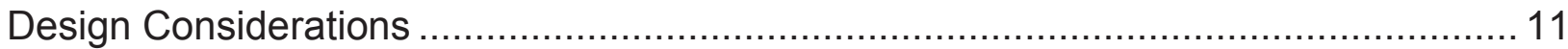

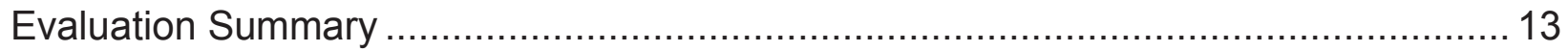

\section{LIST OF FIGURES}

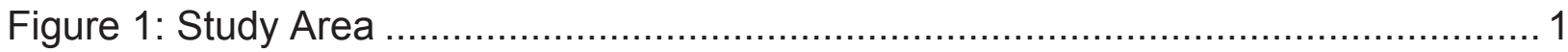

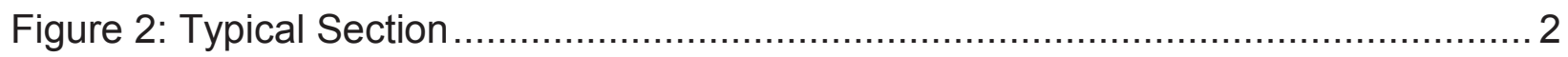

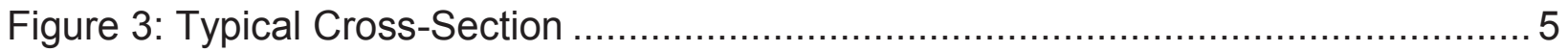

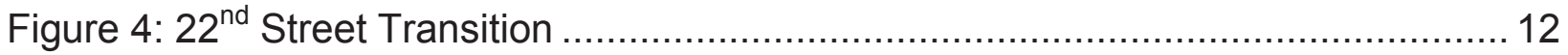

\section{LIST OF TABLES}

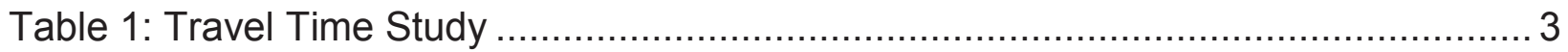

Table 2: Three-Year Crash Summary (2007-2009) .................................................... 4

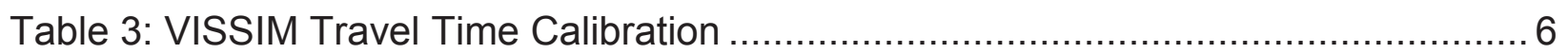

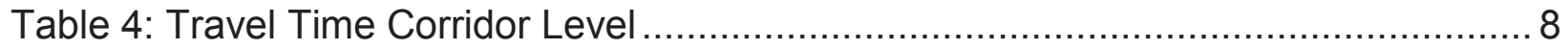

Table 5: PM Peak Hour Intersection Operations Summary ........................................... 9

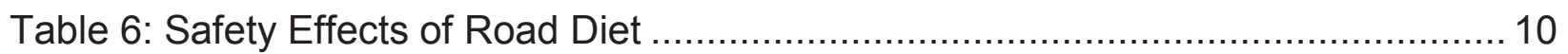

Table 7: Summary of Road Diet Installation Effects ................................................ 13 


\section{INTRODUCTION}

The purpose of this report is to evaluate and present the potential operational and safety impacts associated with the conversion of KY 74 in Middlesboro, Bell County KY from a 4-lane section to a reduced 3-lane section; a treatment referred to as a "road diet." The study area extends from the intersection of $24^{\text {st }}$ Street to the intersection of US 25E (MP 15.712 to MP 16.753); however, the road diet is being considered for the section through downtown Middlesboro from $21^{\text {st }}$ Street to $18^{\text {th }}$ Street. A general location of the study area is shown in Figure 1. This treatment is being considered to increase walkability and serve as a method of traffic calming for traffic traveling through downtown Middlesboro on KY 74.

\section{Figure 1: Study Area}

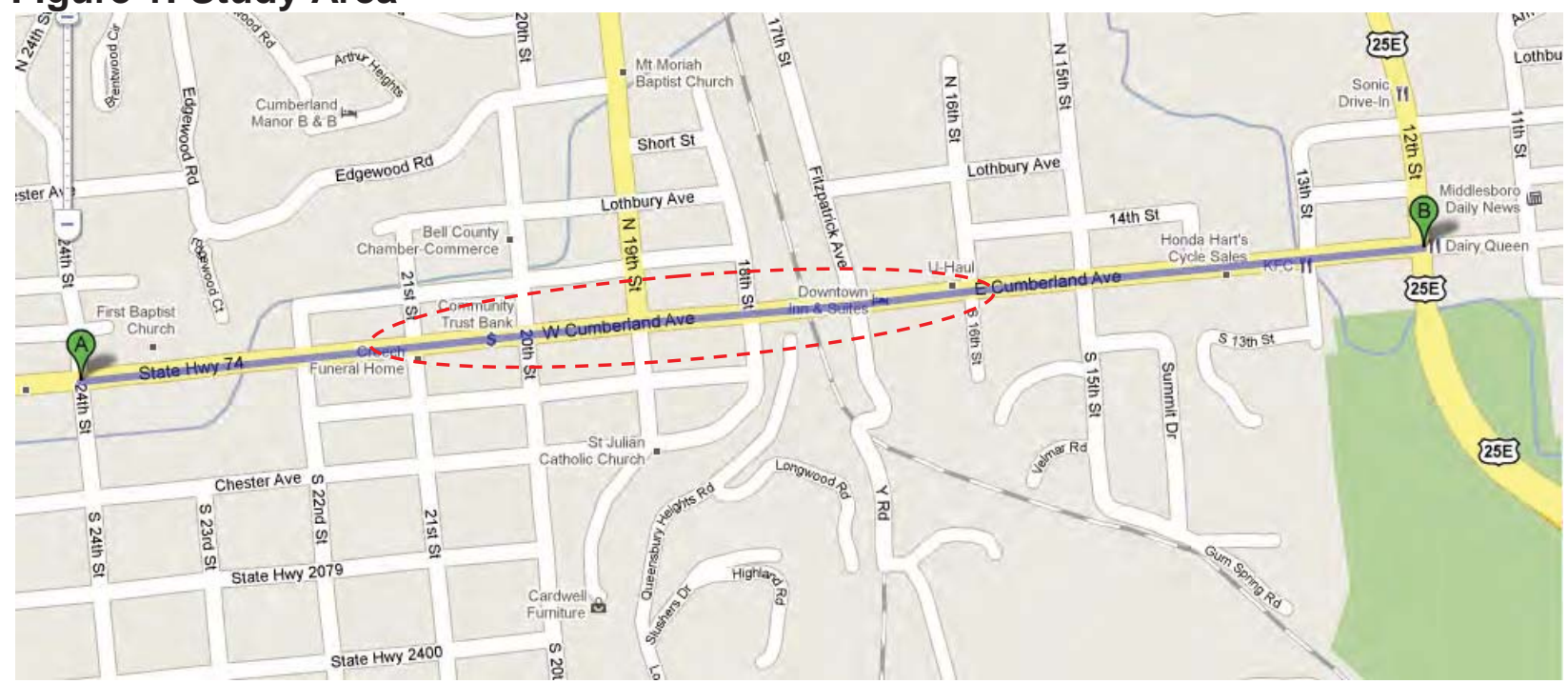

This report summarizes the methodologies used to evaluate the road diet concept and the results of the operational and safety analysis. In addition, special design considerations with the implementation of the treatment on KY 74 are presented and discussed. 


\section{EXISTING CONDITIONS}

The study section KY 74 is characterized as an urban minor arterial with an average daily traffic (ADT) of approximately 12,500 vehicles. The study section has four signalized intersections at $21^{\text {st }}$ Street, $20^{\text {th }}$ Street, $19^{\text {th }}$ Street and $18^{\text {th }}$ Street. The posted speed limit on KY 74 is $35 \mathrm{mph}$.

Through the majority of the study section, KY 74 maintains a 4-lane section with two travel lanes in each direction. Left-turn lanes are provided on KY 74 at South $24^{\text {th }}$ Street and $18^{\text {th }}$ Street. West of $24^{\text {th }}$ Street and east of $18^{\text {th }}$ Street, KY 74 has a 5 -lane section with a full two-way left-turn lane or raised median. Parking is permitted on both sides of the street from $22^{\text {nd }}$ Street to $18^{\text {th }}$ Street. The cross-section is typically 64 feet wide with 12-foot lanes and 8-foot parking lanes. Figure 2 shows the typical section of the corridor.

\section{Figure 2: Typical Section}

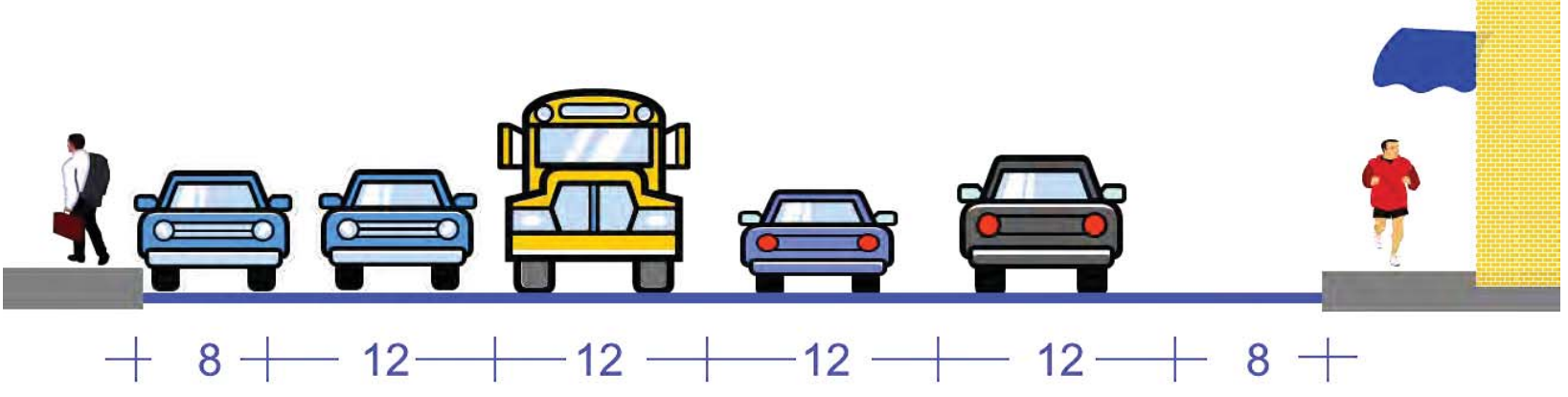

Land use around the study area is primarily commercial as this section of KY 74 represents the commercial center of Middlesboro. There are four signalized intersections and eight commercial access points in the project area. Access points are distributed evenly on the north and south sides of the road.

Appendix A shows the existing lane geometry along the corridor.

\section{Traffic Volumes}

Turn movement counts were conducted by KYTC District 11 for the PM peak period to be used in the evaluation process. This period was chosen by the district as it represents the highest travel demand on the corridor. Appendix B summarizes the peak hour turn movement counts at the signalized intersections.

The ITE Trip Generation Manual was used to estimate turn counts at the unsignalized access points. This process was considered acceptable in estimating the entering to and exiting from the commercial access points, since traffic counts at each point were not available. Trip generation land use codes and facility sizes are summarized in Appendix B. 


\section{Travel Times}

Travel times were collected along the corridor to assist in the calibration of the microsimulation modeling efforts. Average travel times for the corridor are shown in Table 1. The travel times were based on eight runs per direction. The PM peak travel times were 52 seconds for the eastbound and 43 seconds for the westbound direction.

Table 1: Travel Time Study

\begin{tabular}{|c|c|c|c|}
\hline \multirow{2}{*}{$\begin{array}{c}\text { Travel Segment / Direction } \\
\text { (KY 74) }\end{array}$} & \multicolumn{3}{|c|}{ Travel Time (sec) } \\
\hline & AM & Noon & PM \\
\hline \multicolumn{4}{|c|}{ Westbound } \\
\hline 18th St. To 19th St. & 19 & 19 & 15 \\
\hline 19th St. to 20th St. & 17 & 13 & 13 \\
\hline 20th St. to 21st St & 13 & 15 & 15 \\
\hline Total & 48 & 47 & 43 \\
\hline \multicolumn{4}{|c|}{ Eastbound } \\
\hline 21st St. To 20th St. & 15 & 18 & 19 \\
\hline 20th St. To 19th St. & 21 & 14 & 18 \\
\hline 19th St. To 18th St. & 15 & 17 & 15 \\
\hline Total & 51 & 49 & 52 \\
\hline
\end{tabular}

\section{Crash History}

A three-year crash history was collected and analyzed along the corridor. The period evaluated was between January 1, 2007 and December 31, 2009 and there were 135 crashes recorded in this period. The section has a crash rate of 819.9 crashes per 100 million vehicle-miles of travel (MVMT) while the average sate rate for similar roads (fourlane urban arterials) is 475.5 crashes per $100 \mathrm{MVMT}$. The critical crash rate factor for these roads is 617.5 crashes per 100 MVMT and this indicates that the section has a Crash Rate Factor of 1.33 . Table 2 summarizes the crashes by type and location. Crashes are disaggregated by signalized and midblock crashes to assist in identifying crash patterns that may be affected by the implementation of the road diet. Road diets typically can reduce midblock crashes, such as sideswipes and rear ends, as well as intersection crashes, such as angle. However, there is a likelihood of increased number of rear end crashes at intersections. 
Table 2: Three-Year Crash Summary (2007-2009)

\begin{tabular}{|l|c|c|c|}
\hline \multicolumn{1}{|c|}{ Crash Type } & Total & Signalized & Midblock \\
\hline Angle & 48 & 44 & 4 \\
\hline Backing & 5 & 5 & 0 \\
\hline Head on & 2 & 0 & 2 \\
\hline Opposing left turn & 5 & 5 & 0 \\
\hline Rear end & 34 & 24 & 10 \\
\hline Sideswipe-Opposite direction & 3 & 3 & 0 \\
\hline Sideswipe- Same direction & 25 & 11 & 14 \\
\hline Single vehicle & 9 & 5 & 4 \\
\hline Other & 4 & 3 & 1 \\
\hline Total & $\mathbf{1 3 5}$ & $\mathbf{1 0 0}$ & $\mathbf{3 5}$ \\
\hline
\end{tabular}

The data in Table 2 shows that the majority of the crashes on the corridor occur at the signalized intersections. The primary crash type at the signalized intersections is an angle crash, which may be due to the absence of left-turn lanes at the intersections, which will allow for vehicles travelling through seeing left-turning vehicles. This scenario restricts sight distance which can lead to the occurrence of angle crashes.

Crash diagrams showing the location and type of each crash on the corridor are provided in Appendix C. 


\section{ROAD DIET ALTERNATIVE}

The road diet alternative evaluated would reduce the number of lanes from four to three. The new cross section would consist of a single travel lane in each direction and a center two-way left-turn lane. At major intersections the two-way left-turn lane would be operated as a dedicated left-turn lane of the primary intersection. Parallel parking would remain on one side of the road and reverse angle parking will be added on the other side of the roadway. This potential cross-section is shown in Figure 3 . The final configuration should be determined by KYTC in conjunction with the city of Middlesboro to provide the best and safest use of the additional space created by the removal of one lane of travel.

\section{Figure 3: Typical Cross-Section}

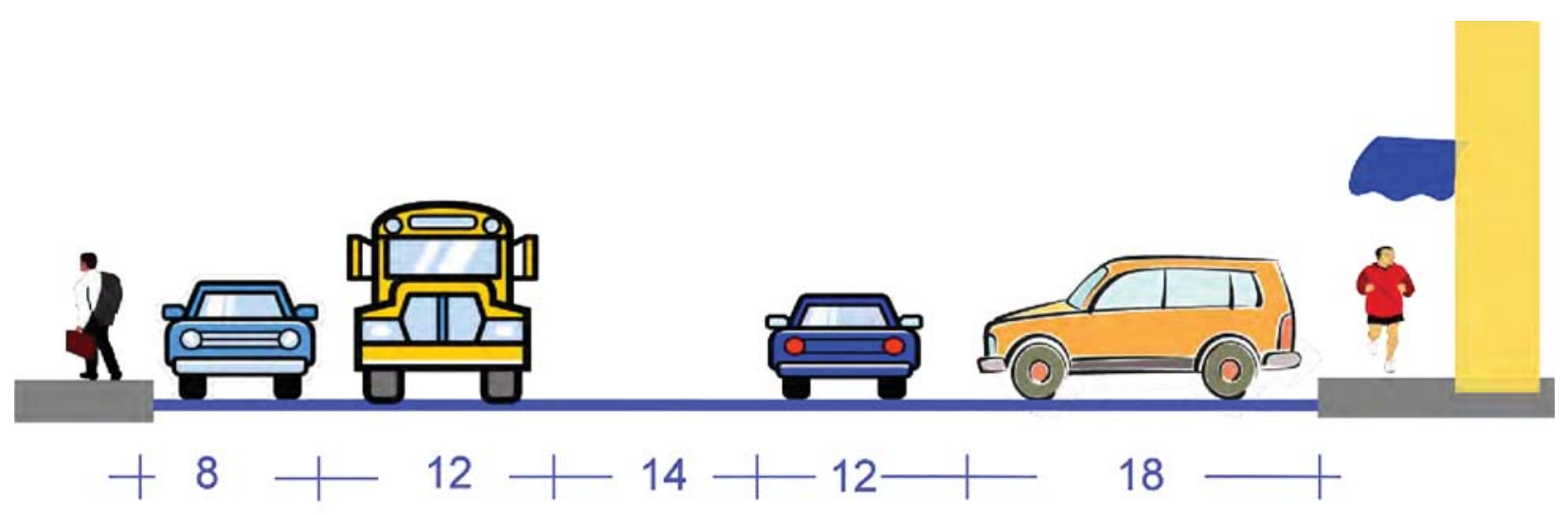




\section{EVALUATION METHODOLOGY}

The road diet on KY 74 was evaluated using the VISSIM 5.1 micro-simulation software. VISSIM is a microscopic simulation model and a component of the PTV Vision $®$ suite. Its flexible network structure provides the ability to model any type of geometric configuration and as such was the preferred software by KYTC to evaluate the operation of a two-way left-turn lane as part of the road diet.

Two models were developed for the corridor. The first was designed to reflect the existing 4-lane cross-section of the corridor. This model was calibrated based on the travel time studies completed by the project team and it represents the existing conditions. The calibration assured that the user characteristics of the model were reflective of drivers and the geometric conditions of the corridor. Based on the calibrated model, an alternative model was developed which assumed implementation of the road diet. Each of the models was evaluated for the AM, Noon and PM peak hours of operations.

The sections below summarize the calibration and measures of effectiveness used to evaluate the VISSIM models.

\section{Calibration}

The existing model was calibrated using the travel time studies (Table 1) in the existing conditions. A threshold of 10 percent above or below the existing travel times was used to determine the effectiveness of the calibrated model. The VISSIM model was calibrated by adjusting turning volumes, turning speeds and travel speeds through the corridor. Table 3 summarizes the travel time calibration for the KY 74 corridor.

Table 3: VISSIM Travel Time Calibration

\begin{tabular}{|l|c|c|c|c|c|c|}
\hline \multirow{2}{*}{$\begin{array}{c}\text { Travel Segment } \\
\text { (KY 74) }\end{array}$} & \multicolumn{2}{|c|}{ AM } & \multicolumn{2}{c|}{ Noon } & \multicolumn{2}{c|}{ PM } \\
\cline { 2 - 7 } & Field & VISSIM & Field & VISSIM & Field & VISSIM \\
\hline \multicolumn{7}{|c|}{ Westbound } \\
\hline 18th St. To 19th St. & 19 & 17 & 19 & 18 & 15 & 18 \\
\hline 19th St. to 20th St. & 17 & 14 & 13 & 16 & 13 & 18 \\
\hline 20th St. to 21st St & 13 & 16 & 15 & 14 & 15 & 18 \\
\hline Total & $\mathbf{4 8}$ & $\mathbf{4 7}$ & $\mathbf{4 7}$ & $\mathbf{4 9}$ & $\mathbf{4 3}$ & $\mathbf{5 4}$ \\
\hline \multicolumn{7}{|c|}{ Eastbound } \\
\hline 21st St. To 20th St. & 15 & 14 & 18 & 15 & 19 & 15 \\
\hline 20th St. To 19th St. & 21 & 17 & 14 & 18 & 18 & 22 \\
\hline 19th St. To 18th St. & 15 & 19 & 17 & 18 & 15 & 22 \\
\hline Total & $\mathbf{5 1}$ & $\mathbf{5 0}$ & $\mathbf{4 9}$ & $\mathbf{5 1}$ & $\mathbf{5 2}$ & $\mathbf{5 9}$ \\
\hline
\end{tabular}

The calibration is within the acceptable 10 percent variation. Moreover, this calibration and recordings of the VISSIM model were provided to District 11 and approved as accurately representing the existing conditions on the corridor. 


\section{Measures of Effectiveness}

Operational performance of the road diet alternative was evaluated for both corridor operations as well as individual signalized intersections on the corridor. Corridor operations were evaluated using travel times through the entire section. Intersection operations were evaluated using the following four measures of effectiveness, which are for the entire intersection.

- Delay (Seconds)

- Stopped Delay (Seconds)

- Number of Stops

- Average Queue Length (feet) 


\section{RESULTS}

\section{Operational Analysis}

Table 4 summarizes the results of corridor level analysis for the implementation of a road diet on KY 74. The data in the table indicates that there is a slight decrease in travel times after the implementation of the road diet. This decrease is between 1 to 9 seconds during the peak periods, representing approximately a 2 to 18 percent decrease in travel times.

Table 4: Travel Time Corridor Level

\begin{tabular}{|l|c|c|c|c|c|c|}
\hline \multirow{2}{*}{$\begin{array}{c}\text { Travel Segment } \\
\text { (KY 74) }\end{array}$} & \multicolumn{7}{|c|}{ AM } & \multicolumn{2}{c|}{ Noon } & \multicolumn{2}{c|}{ PM } \\
\cline { 2 - 7 } & 4-Lane & 3-Lane & 4-Lane & 3-Lane & 4-Lane & 3-Lane \\
\hline \multicolumn{7}{|c|}{ Westbound } \\
\hline 18th St. To 19th St. & 17 & 11 & 18 & 11 & 18 & 14 \\
\hline 19th St. to 20th St. & 14 & 15 & 16 & 14 & 18 & 16 \\
\hline 20th St. to 21st St & 16 & 15 & 14 & 15 & 18 & 19 \\
\hline Total & $\mathbf{4 7}$ & $\mathbf{4 1}$ & $\mathbf{4 9}$ & $\mathbf{4 0}$ & $\mathbf{5 4}$ & $\mathbf{4 9}$ \\
\hline \multicolumn{7}{|c|}{ Eastbound } \\
\hline 21st St. To 20th St. & 14 & 18 & 15 & 18 & 15 & 20 \\
\hline 20th St. To 19th St. & 17 & 15 & 18 & 15 & 22 & 19 \\
\hline 19th St. To 18th St. & 19 & 16 & 18 & 16 & 22 & 15 \\
\hline Total & $\mathbf{5 0}$ & $\mathbf{5 0}$ & $\mathbf{5 1}$ & $\mathbf{5 0}$ & $\mathbf{5 9}$ & $\mathbf{5 4}$ \\
\hline
\end{tabular}

Table 5 summarizes the intersection delay and queue estimates for the PM peak hour. The overall pattern in the data presented in Table 5 indicates no significant changes in delays for the PM peak period after the installation of the road diet. Intersection delays will increase by 1 to $2 \mathrm{sec} / \mathrm{veh}$ at the various intersections representing an increase between 3 to 19 percent increase. Queue lengths at all intersections are reduced significantly-for three of the four at about one-third of the original length. This could be attributed to the presence of the left-turn auxiliary lane at the intersections separating left-turning and through movements. An issue that should be noted here is the lack of significant changes in delays and significant improvements in queue lengths indicating that the road diet application will not impact negatively operations. 
Table 5: PM Peak Hour Intersection Operations Summary

\begin{tabular}{|l|c|c|c|c|}
\hline \multirow{2}{*}{ Intersection } & \multicolumn{2}{|c|}{ Delay (sec) } & \multicolumn{2}{c|}{ Average Queue (ft) } \\
\cline { 2 - 5 } & Existing & Road Diet & Existing & Road Diet \\
\hline KY 74 at 21st Street & 10.09 & 12.06 & 12.98 & 5.64 \\
\hline KY 74 at 20th Street & 9.95 & 10.89 & 17.74 & 5.14 \\
\hline KY 74 at 19th Street & 13.09 & 14.42 & 24.63 & 7.89 \\
\hline KY 74 at 18th Street & 29.38 & 30.56 & 43.55 & 14.39 \\
\hline
\end{tabular}

\section{Safety Analysis}

The primary crash type on the corridor is an angle crash at an intersection (Table 2). This crash type is likely caused by the absence of left-turn lanes that could provide unrestricted sight distance to oncoming traffic. The implementation of a left-turn lane at these intersections through the road diet will correct this problem and significantly improve sight distance for left-turning vehicles. This should result in a reduction of angle crashes.

The second most frequent crash type on the corridor is rear end crashes and most of they are occurring at intersections. This crash type is typically attributed to a combination of the lack of left-turn lanes (causing rear end crashes when a vehicle stops to turn left) and congestion on the corridor. The operational analysis presented in Tables 4 and 5 indicate that there will be no changes in the level of congestion and therefore no changes are anticipated in crashes resulting from congestion. However, the introduction of left-turn lanes and the separation of through and left-turning vehicles will likely have a positive effect of reducing some of the rear end crashes at the signalized intersections. It should be pointed though that this effect will not apply at those intersections which already have left-turn lanes.

Approximately 30 percent of the crashes are at midblock locations and the road diet can potentially address several of these crashes. Rear end and sideswipes are the two primary crash types that road diets typically reduce by eliminating the friction created by stopped left-turning vehicles in traffic. The removal of left-turning vehicles from the main stream of traffic eliminates unexpected stopped vehicles that could lead to rear ends or the need to swerve to avoid the stopped vehicles resulting in sideswipes. Table 6 summarizes the potential effects of road diet implementation on crashes for KY 74 . 
Table 6: Safety Effects of Road Diet

\begin{tabular}{|l|c|c|c|c|}
\hline \multirow{2}{*}{\multicolumn{1}{|c|}{ Crash Type }} & \multicolumn{2}{c|}{ Signalized } & \multicolumn{2}{c|}{ Midblock } \\
\cline { 2 - 5 } & Number & Effect & Number & Effect \\
\hline Angle & 12 & - & 1 & - \\
\hline Backing & 1 & & 0 & \\
\hline Head on & 0 & & 1 & - \\
\hline Opposing left turn & 1 & - & 0 & \\
\hline Rear end & 7 & $+/-$ & 3 & - \\
\hline Rear to rear & 1 & & 0 & - \\
\hline Sideswipe-Opposite direction & 2 & & 0 & \\
\hline Sideswipe- Same direction & 4 & - & 5 & - \\
\hline Single vehicle & 2 & & 2 & \\
\hline Total & $\mathbf{2 7}$ & - & 11 & - \\
\hline Legend: (-) indicates reduction in crashes; (+/-) indicates mixed impact based on causal factors of the crash. \\
\hline
\end{tabular}




\section{DESIGN CONSIDERATIONS}

Two primary considerations need to be addressed with respect to road diet implementation. The first is to identify the preferred utilization of the additional space created by the reduction in travel lanes. The proposed cross sections shown in Figure 3 provide a preliminary recommendation for the utilization of the additional space. For most sections of the project, the extra space can be used to provide additional parking opportunity in both sides of the corridor. In this case, 12-foot lanes will be implemented with a 14-foot two-way left-turn lane and an 8-foot parking strip in one side and an 18foot reverse angle parking in the other side.

The second consideration is to properly locate and design the transition from the existing 4- and 5-lane sections to the reduced 3-lane section. For the east end of the corridor, the transition should occur at the $22^{\text {nd }}$ and $23^{\text {rd }}$ Streets. For the west end of the corridor, the transition should occur at the $18^{\text {th }}$ Street. The additional inbound lane to the study corridor is recommended to be dropped as a right-turn lane at these intersections. A conceptual plan for the transition at $22^{\text {nd }}$ and $23^{\text {rd }}$ Streets is shown in Figure 4. 


\section{EVALUATION SUMMARY}

The analysis presented here evaluates the feasibility of converting the 4-lane section of KY 74 in Middlesboro to a 3-lane cross-section with one lane in each direction and a two-way left-turn lane. The simulation models developed in VISSIM were calibrated to reflect the field conditions. The analysis completed evaluated the operational and safety effects of this potential change. A summary of the overall potential effects of the installation is shown in Table 7.

Table 7: Summary of Road Diet Installation Effects

\begin{tabular}{|l|l|}
\hline \multicolumn{1}{|c|}{ Aspect } & \multicolumn{1}{c|}{ Effect } \\
\hline Travel times & None or negligent \\
\hline Intersection delays & None or negligent \\
\hline Overall safety & $\begin{array}{l}\text { Improved; reduced conflict points; reduced } \\
\text { angle and sideswipe crashes; potential to } \\
\text { increase rear end crashes at intersections }\end{array}$ \\
\hline Travel speeds & Improved uniformity; traffic calming potential \\
\hline Pedestrian safety & Improved \\
\hline Multimodal operations & Improved for bicycles and transit \\
\hline Access management & Improved potential \\
\hline
\end{tabular}

The operational analysis showed that travel times will remain virtually unchanged with the conversion. The comparison of travel times from Table 4 shows a decrease of approximately 10 percent for both directions in the PM peak hours (along with lower reductions in the other peak periods). All signalized intersections along the corridor will experience a slight increase in overall delays indicating that the conversion will have no negative effects on the operation of the corridor. Queue lengths at the signalized intersections will be significantly reduced to approximately one-third of current lengths. It should be noted that there are additional benefits that could materialize from the conversion that the simulation was not able to quantify. These benefits include the potential for more uniform speeds along the corridor, elimination of unbalanced speeds by travel lanes and potential speed reductions (road diet is acting as traffic calming). Additionally, pedestrian comfort and safety may be improved by reducing the number lanes to be crossed, providing a refuge within the two-way left-turn lane and reducing the speed differential in the travel lanes. Road diets also improve multimodal operations (bicycles and transit) through the presence of either wider lanes or exclusive bicycle lanes as well as improve access management potential.

The safety analysis showed that there is the potential for improvements both at the signalized intersections and midblock areas along the corridor. The road diet installation is expected to reduce angle and sideswipe crashes due to the removal of the left-turning vehicles for the travel lanes. Rear end crashes at midblock sections are also expected to reduce while rear end crashes at intersections could be affected as well but there is also the potential of increasing them with the placement of the left-turn lanes at the intersections. The road diet installation can therefore reduce the number of crashes and 
thus impact the crash rate factor if 1.33 noted for this section. Additional safety gains that could materialize from road diet installations include reduced number of conflict points, improved pedestrian safety (through provision of median refuge), and potential for lower severity crashes due to reduced travel speeds.

One potential disadvantage of the road diet would be the ability to handle disruptions in the traffic flow resulting from incidents. The reduced number of travel lanes could impact operations if one lane is blocked through a crash occurrence or other intermittent incidents, such as train movements. During these abbreviated times, queues are expected to be longer, potentially impacting side street operations. The longer queues would also take longer to dissipate after an incident. 


\section{APPENDIX A}

Existing Conditions

Road Diet Case Study Evaluation

KY 74, Middlesboro, KY 


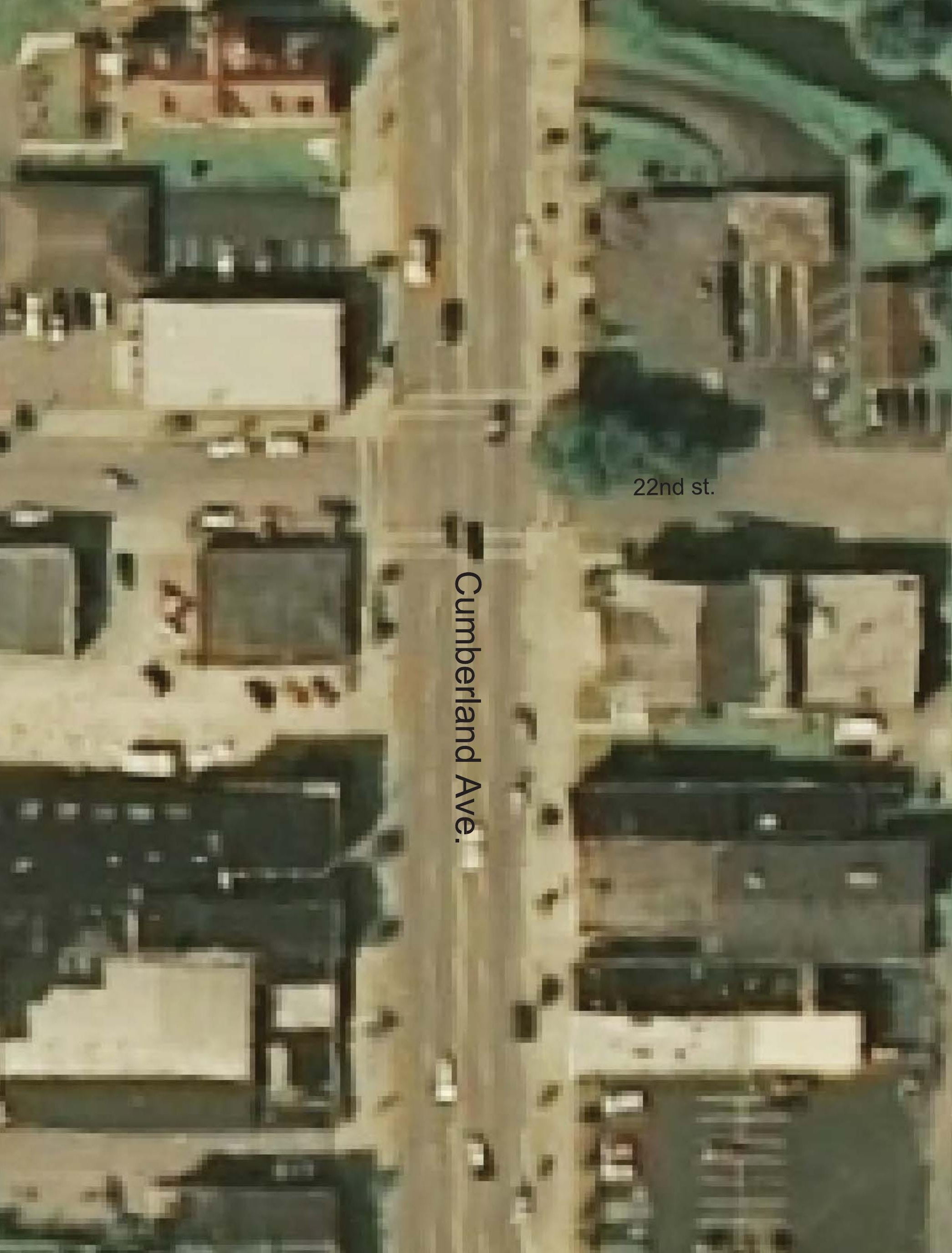




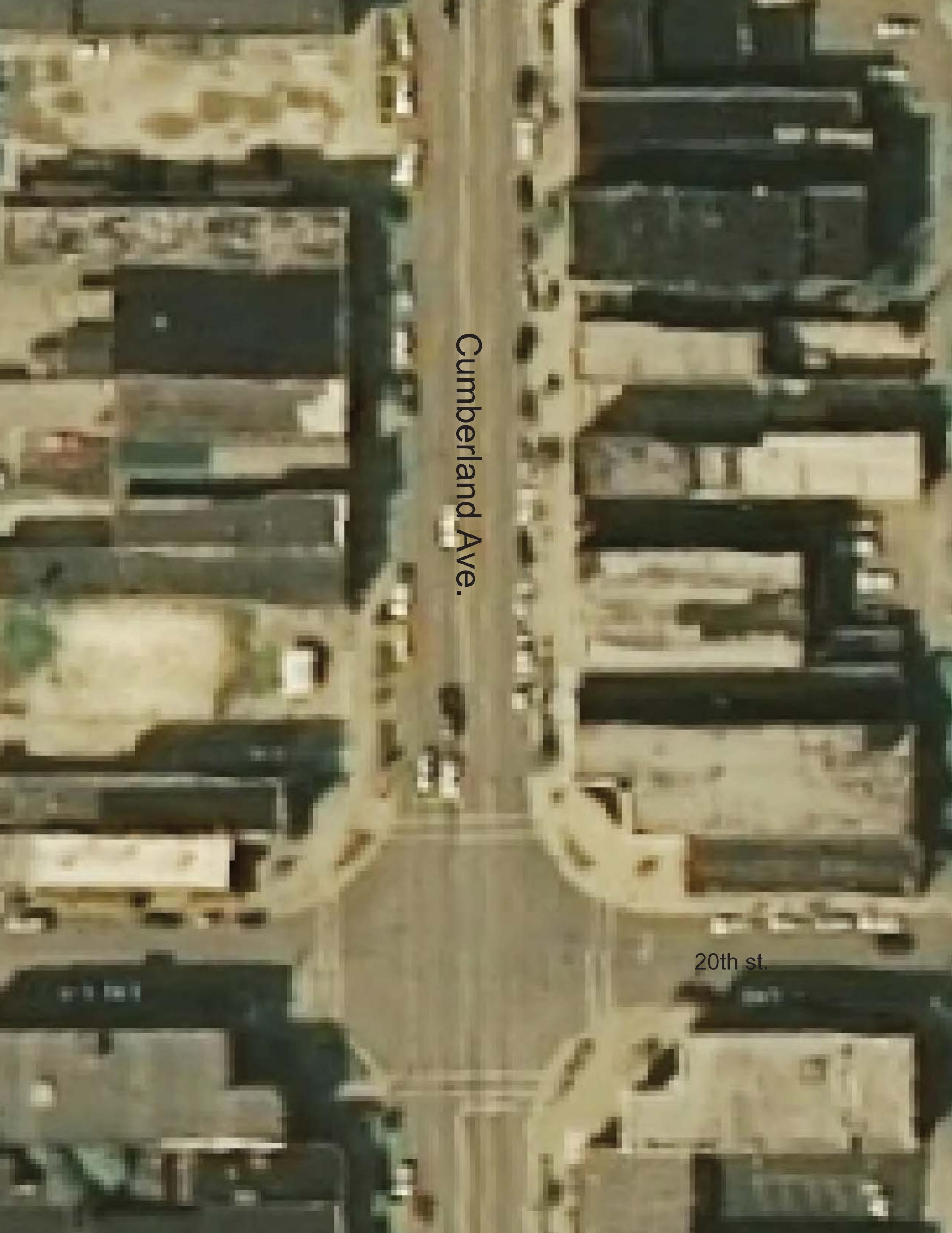




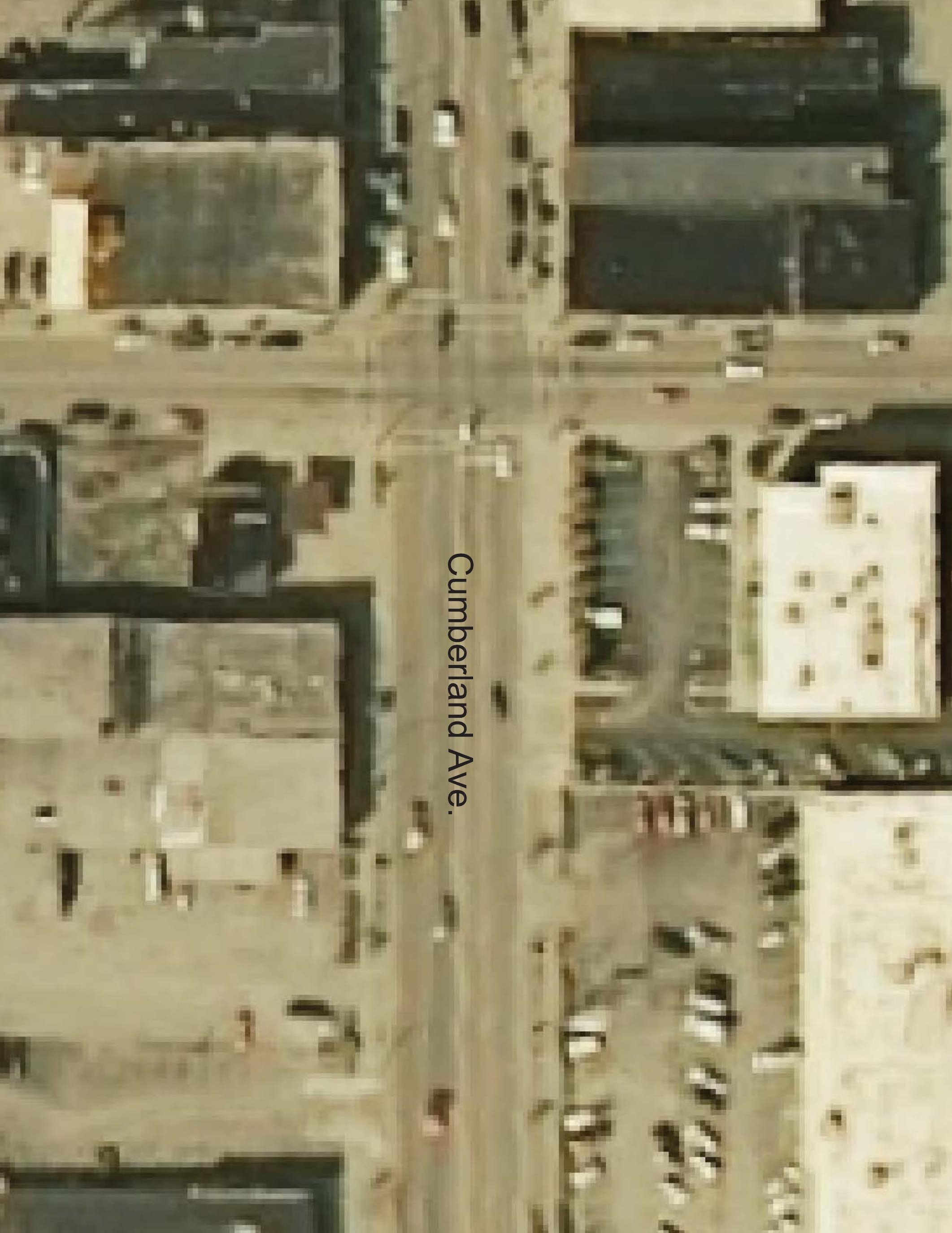




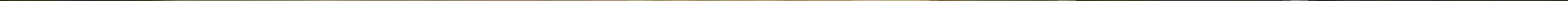




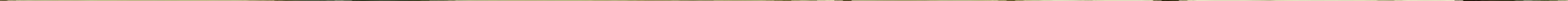




\section{APPENDIX B}

Traffic Volumes

Road Diet Case Study Evaluation

KY 74, Middlesboro, KY 

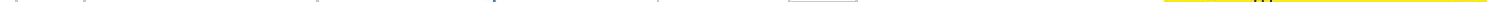


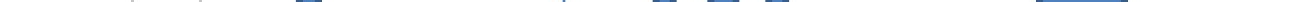




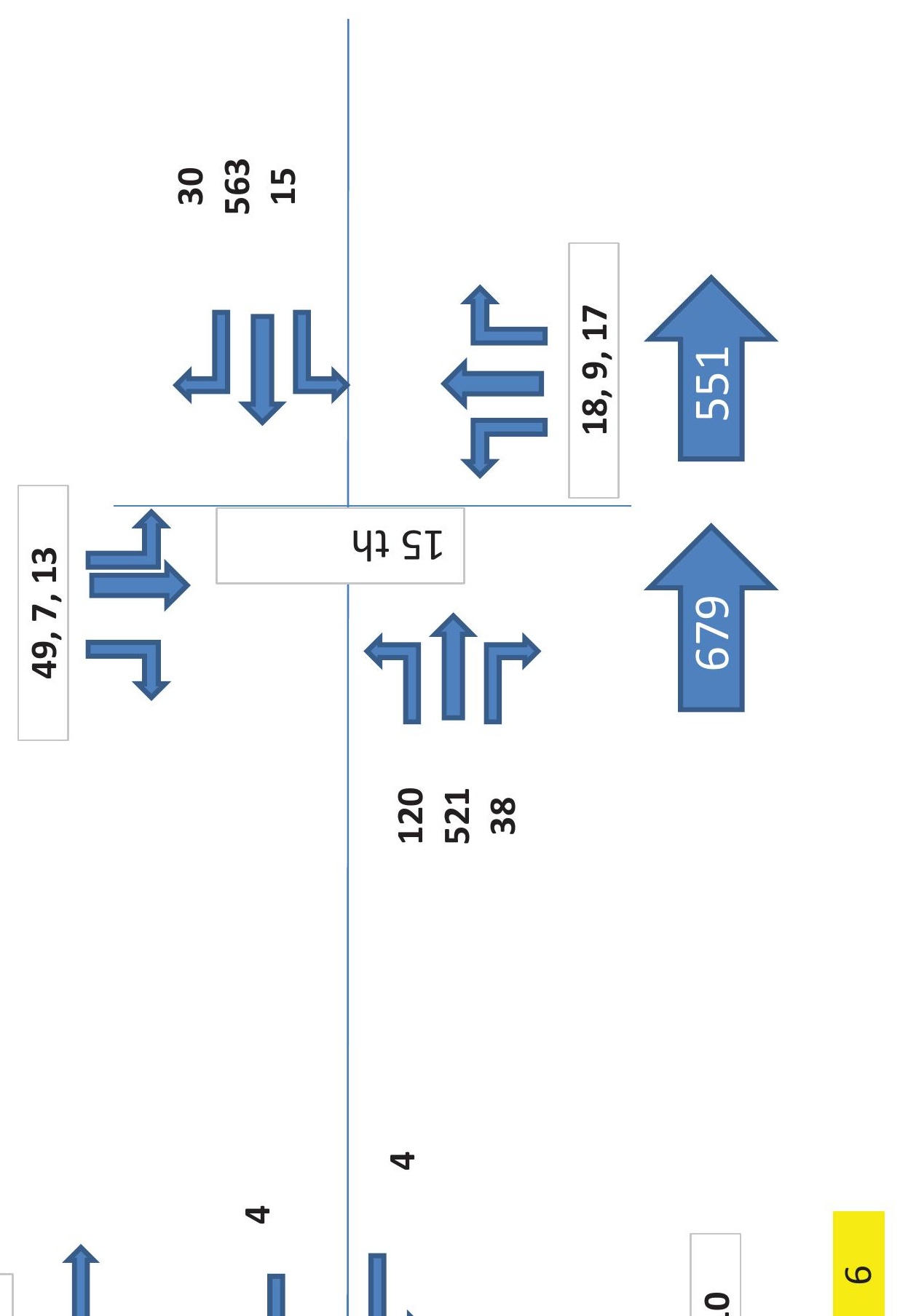




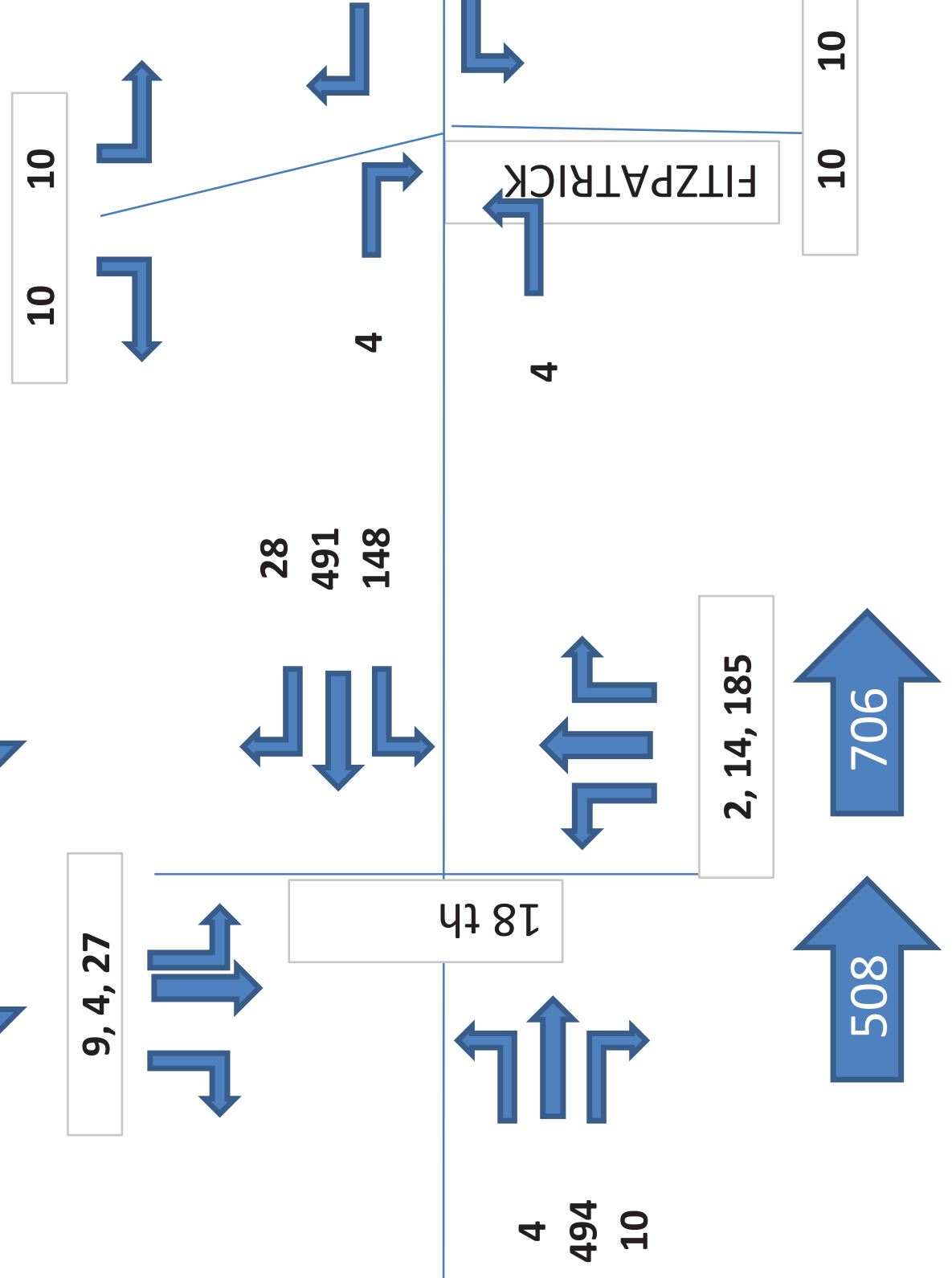

$\mathbf{V}$
$\Omega$

+
+
$\mathbf{1}$

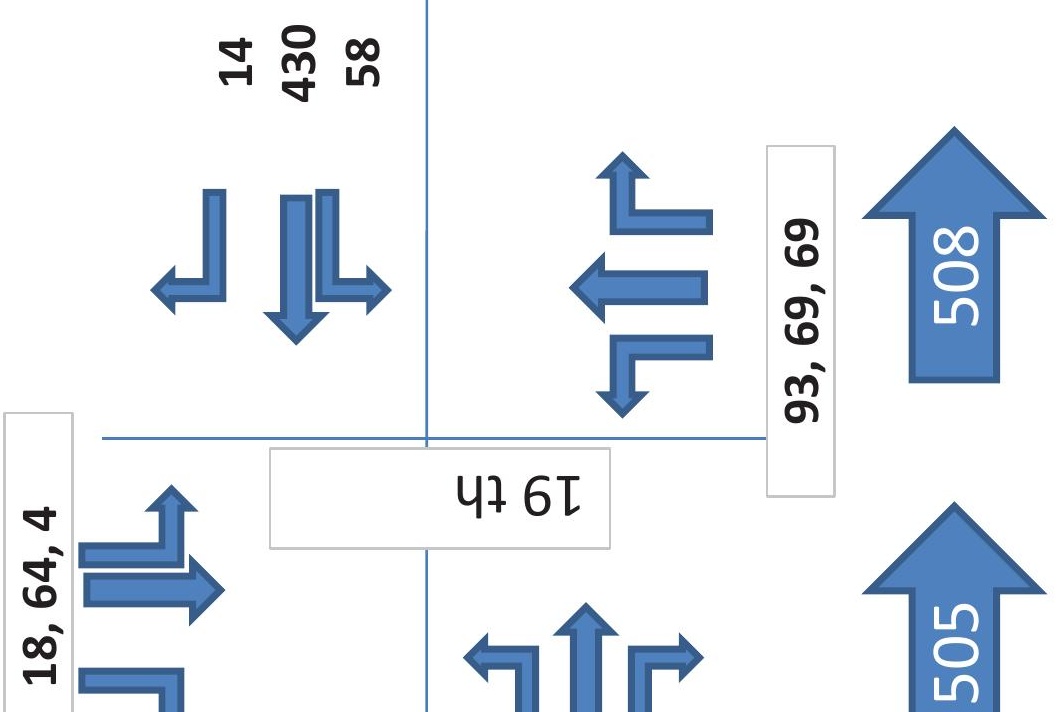




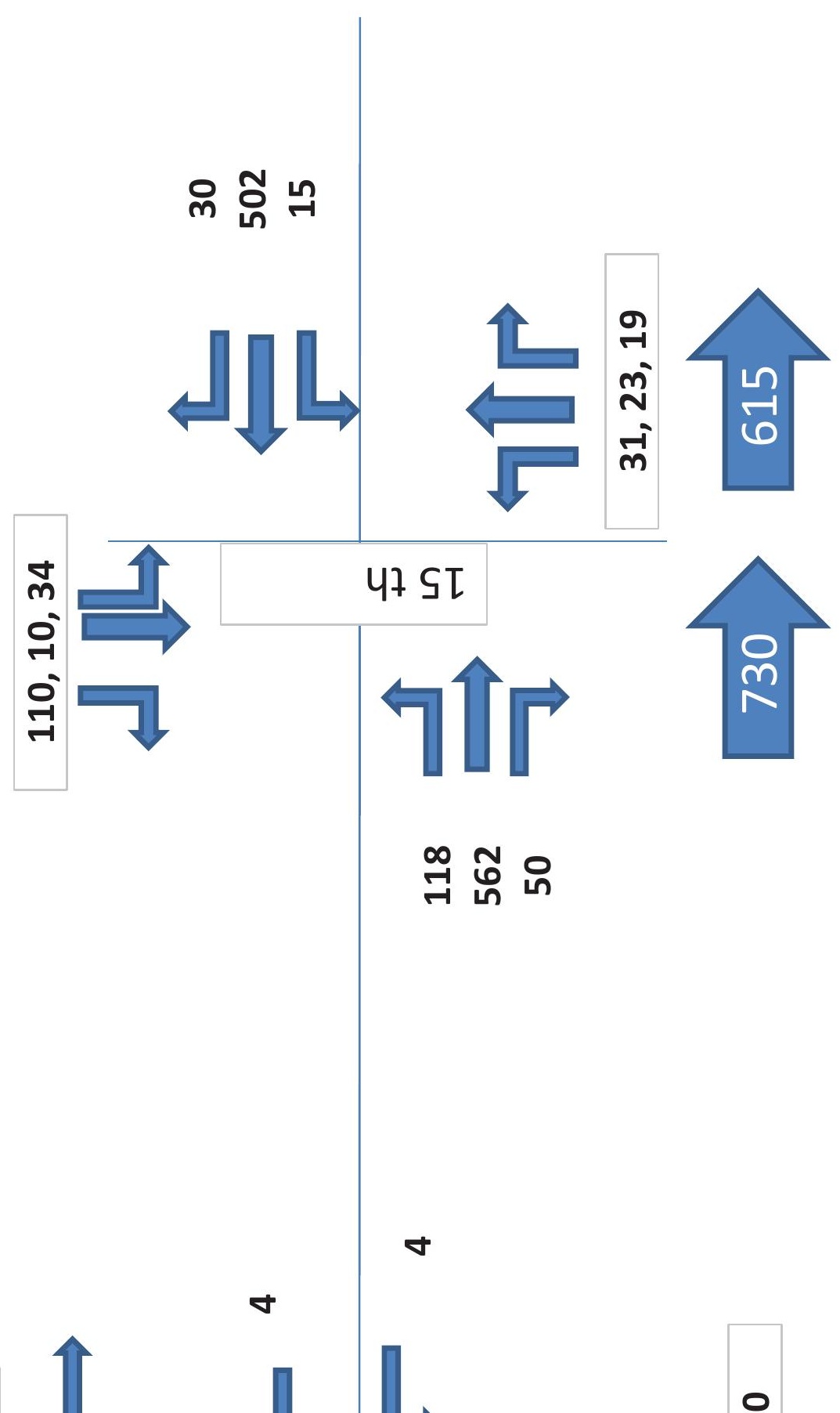



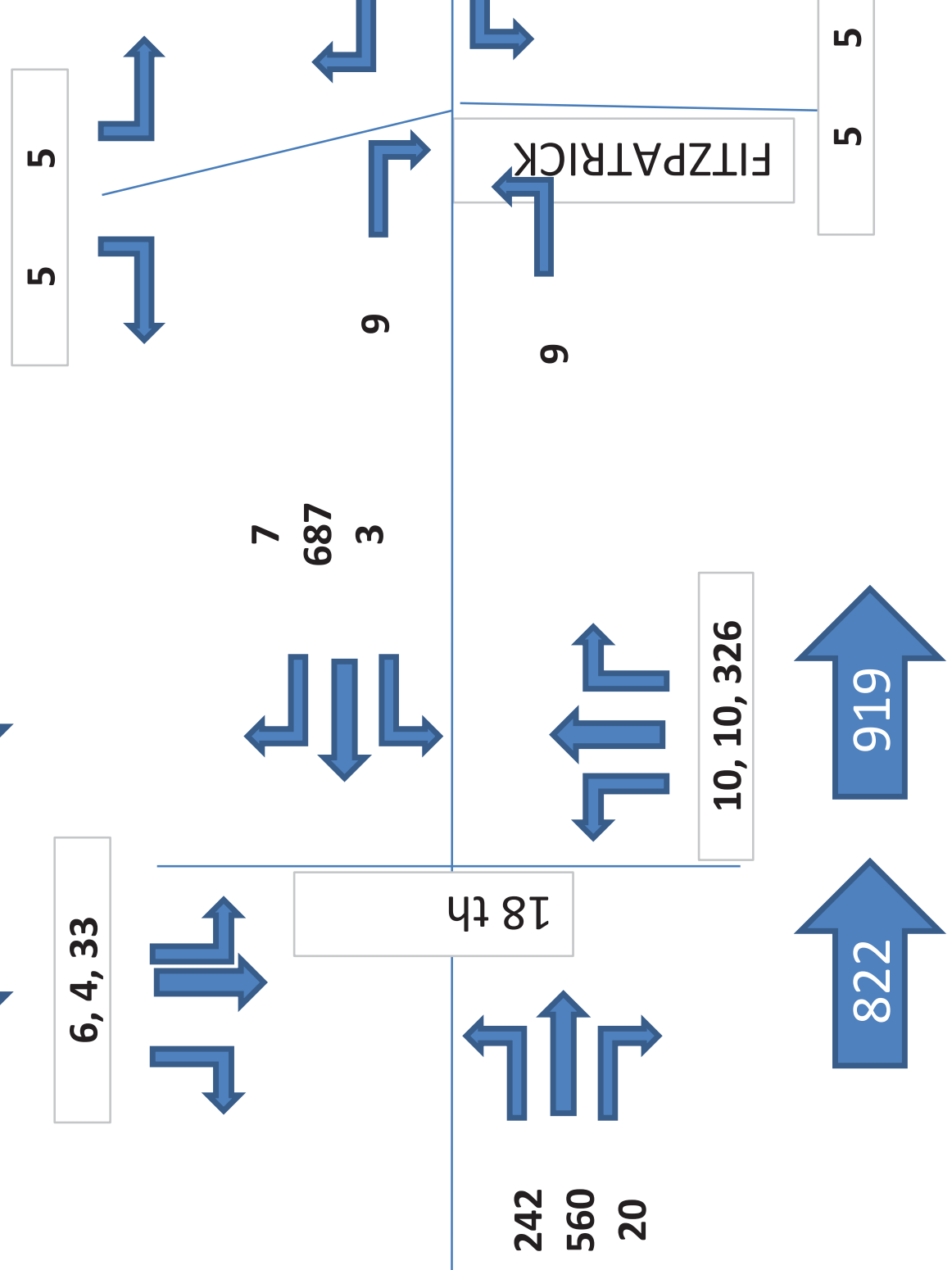

싱 ํㅜㄱ
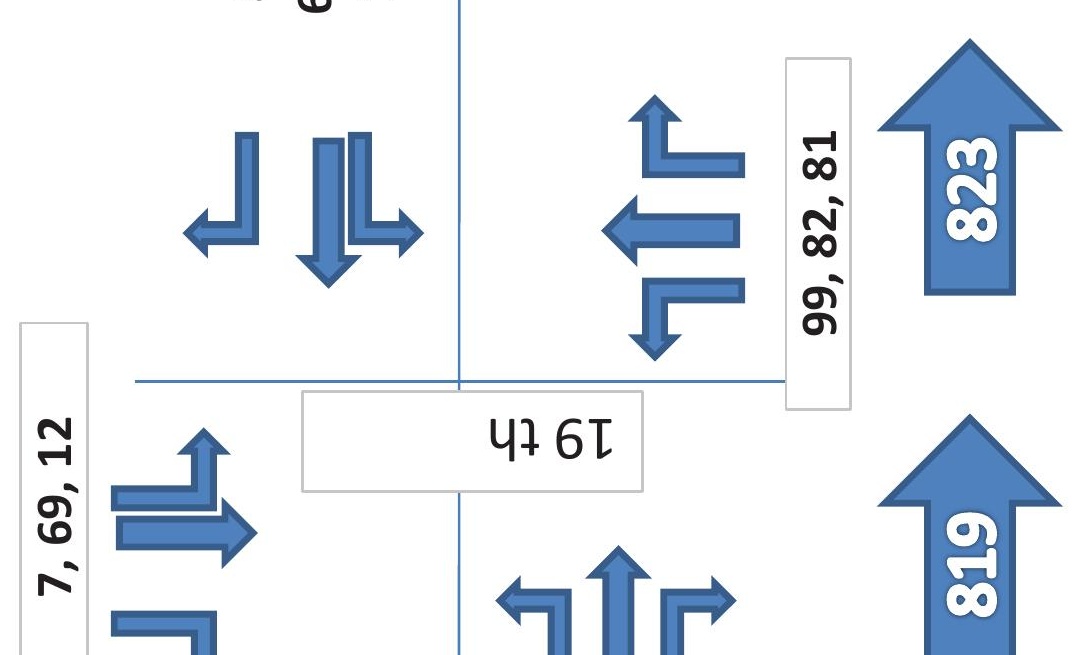


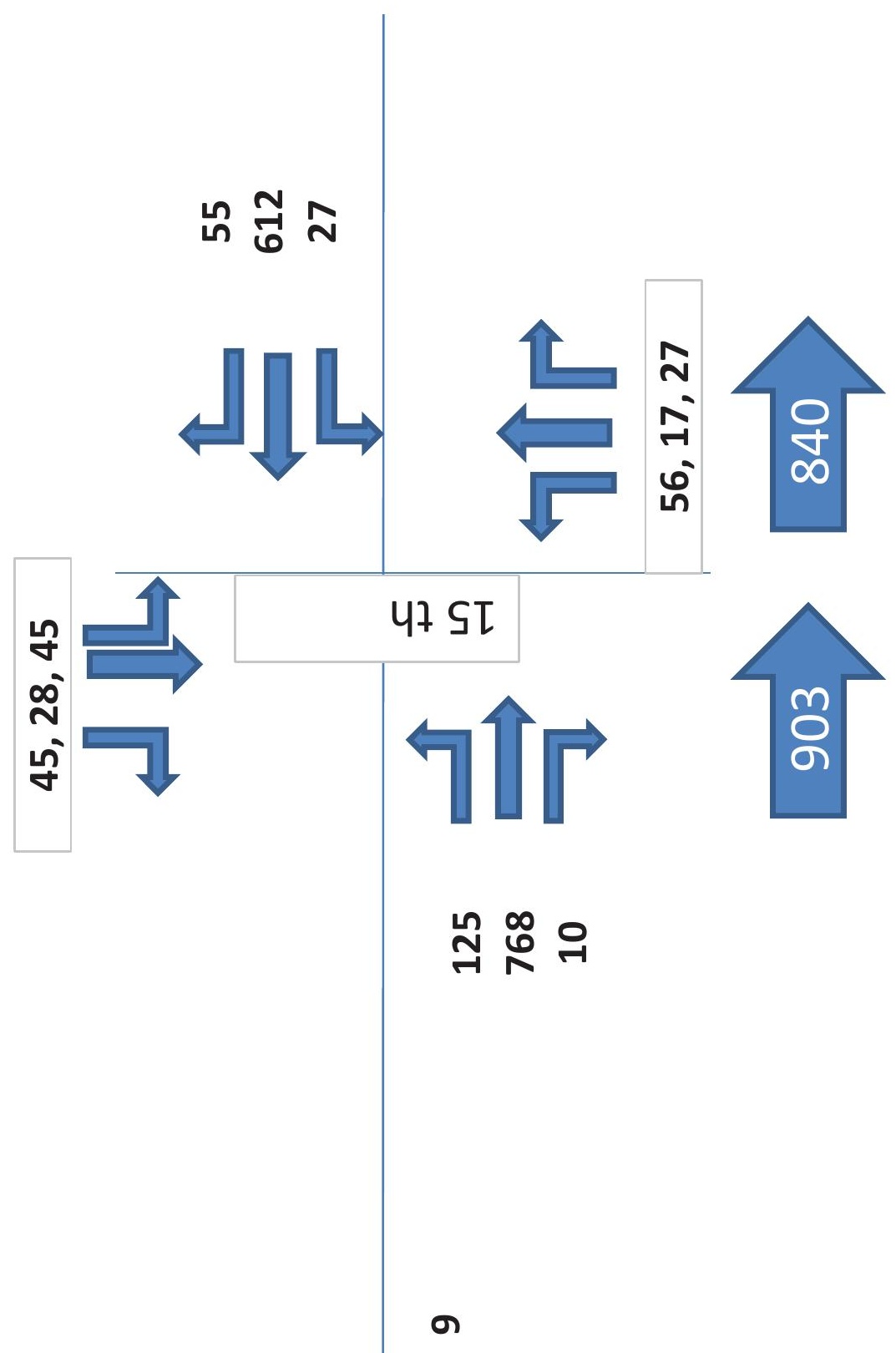




\section{APPENDIX C \\ Crash Diagrams}

Road Diet Case Study Evaluation

KY 74, Middlesboro, KY 
$\Omega$

3

D

a)

0

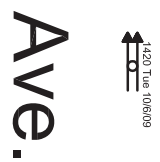




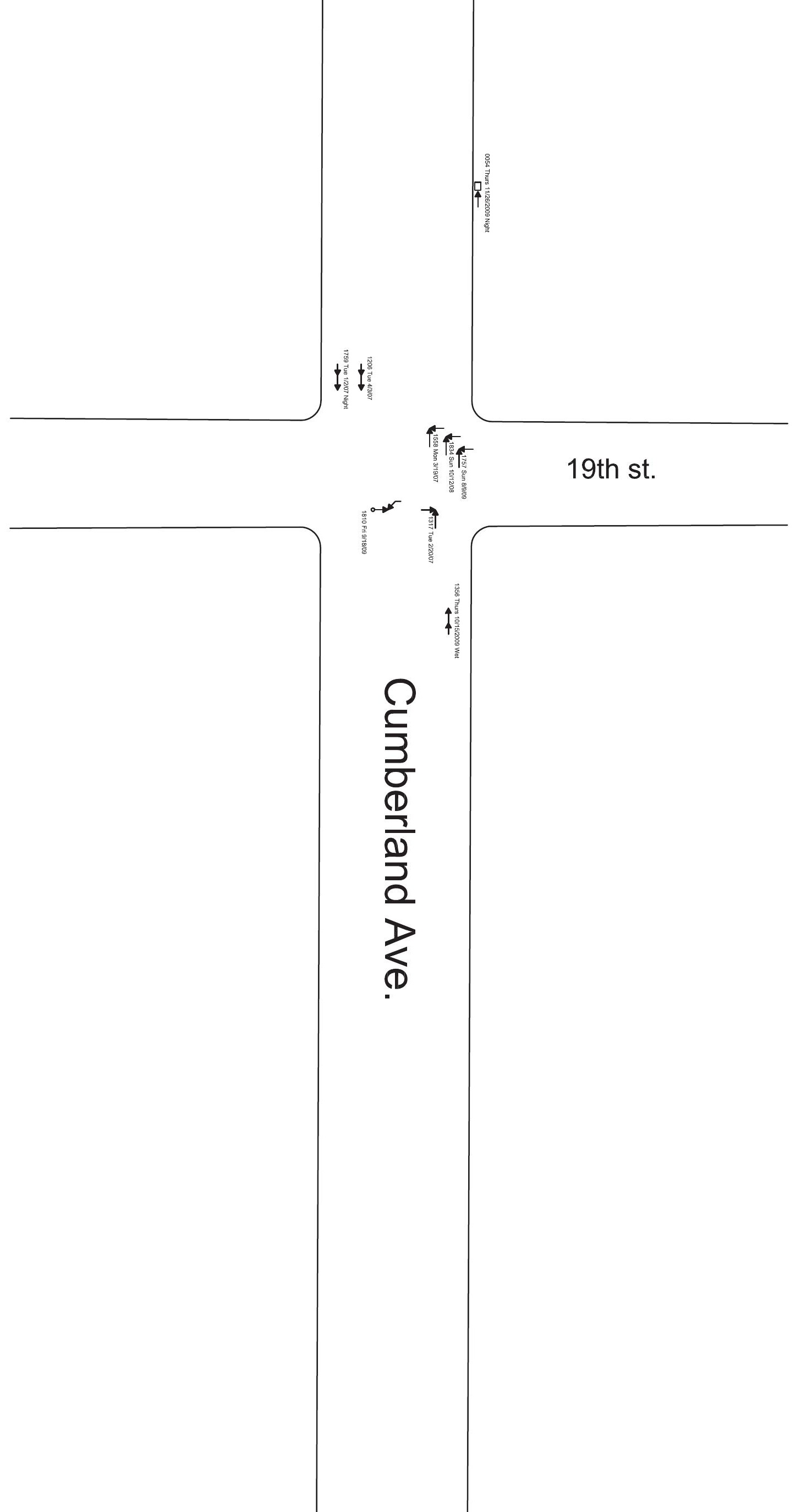


16th St.

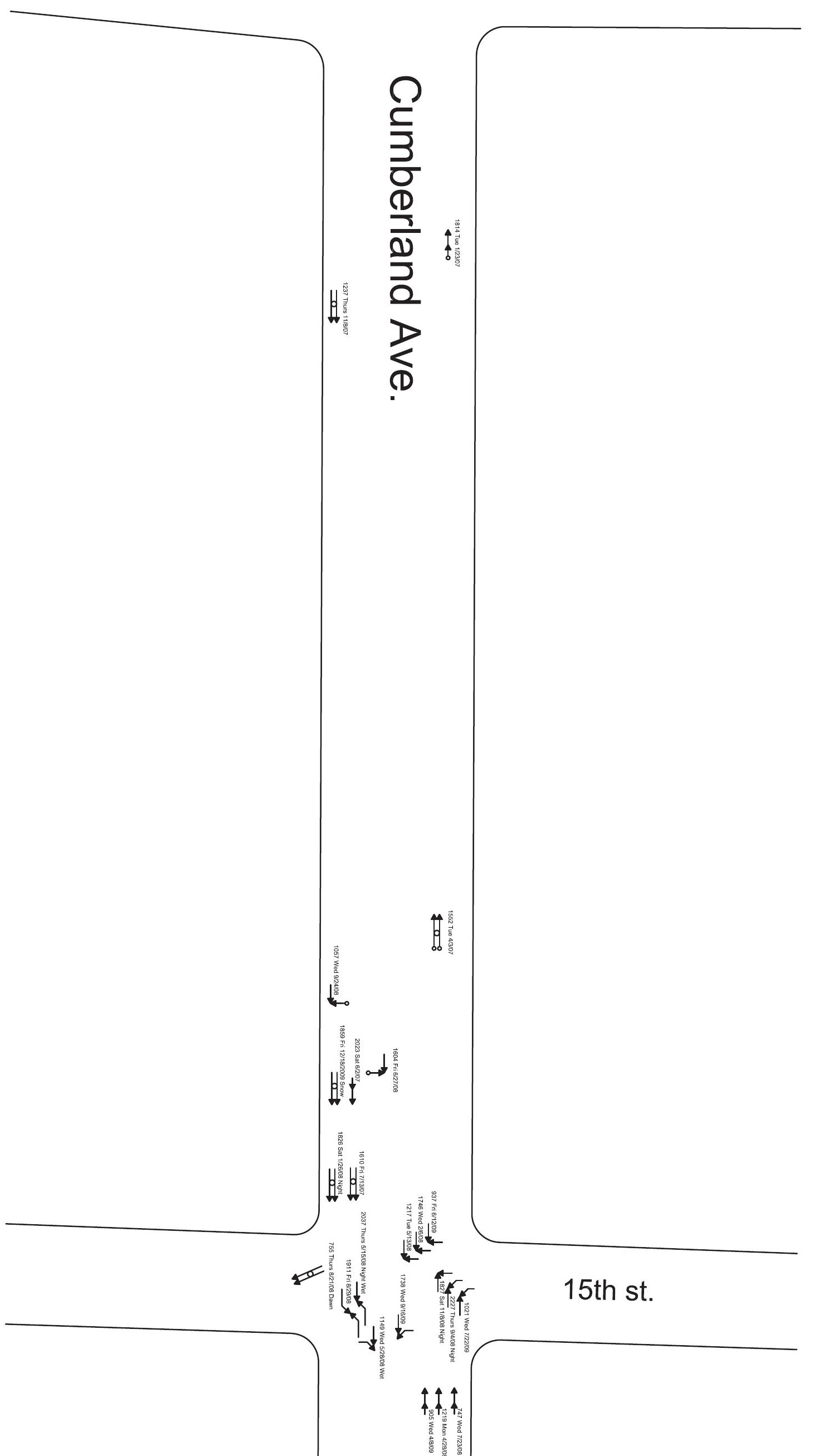


APPENDIX B

Road Diet Guidelines 


\section{INTRODUCTION}

Road diets usually involve restriping a four-lane undivided road as a three-lane road with two through lanes and a two-way left-turn lane. The extra roadway width can be used to widen existing lanes, create bicycle lanes, supply on street parking, widen sidewalks, or provide opportunities for landscaped median islands.

Typical considerations for road diet conversions include traffic volumes of the minor and main streets and main street left-turn percentages. However, compatibility of the treatment with roadway functional classification, access frequency and land use should also be considered when determining the feasibility of a road.

\section{ROAD DIET CONVERSIONS}

\section{Process}

Road diets may be proposed to address both safety and operational issues on roadways. The following flow chart provides an overview of the process to be followed to determine whether a road diet is appropriate (Figure 1).

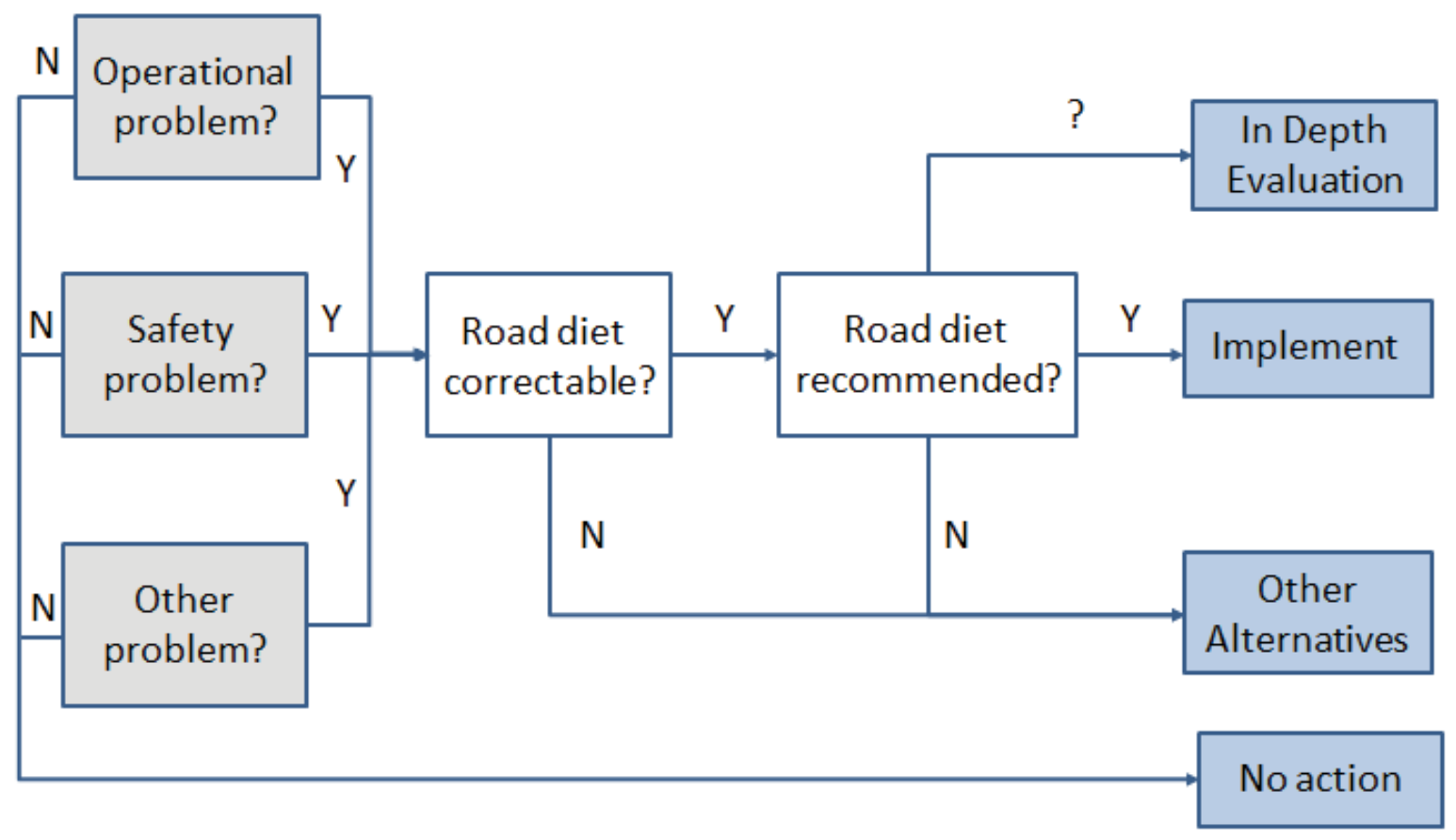

Figure 1 Decision-action flow chart for road diet evaluations

The flow chart allows the user to identify the appropriate action to be undertaken in order to determine whether the road diet will improve the operations, safety or other performance issues associated with the road. To address this, a list of possible problems correctable by a road diet implementation is presented below (Table 1). In addition, the required items for conducting an 
in-depth evaluation are also identified in order to allow for a complete evaluation of the implications from a road diet implementation (Table 2).

Table 1 Road diet correctable problems

\begin{tabular}{|c|c|c|}
\hline Category & Problem & Rationale \\
\hline \multirow{3}{*}{ Operational } & $\begin{array}{l}\text { Delays associated with left- } \\
\text { turning traffic }\end{array}$ & $\begin{array}{l}\text { Separation of left-turning traffic have shown to } \\
\text { improve delays at signalized intersections }\end{array}$ \\
\hline & $\begin{array}{l}\text { High side street delays at } \\
\text { unsignalized intersections }\end{array}$ & $\begin{array}{l}\text { Side street traffic requires shorter gaps to } \\
\text { complete movements due to the consolidation } \\
\text { of left-turns into one lane }\end{array}$ \\
\hline & Bicycle delays & $\begin{array}{l}\text { Potential for including a bike lane eliminates } \\
\text { such delays }\end{array}$ \\
\hline \multirow{4}{*}{ Safety } & $\begin{array}{l}\text { Rear end crashes with left- } \\
\text { turning traffic }\end{array}$ & $\begin{array}{l}\text { Removal of stopped turning vehicles from the } \\
\text { through lane could reduce rear end crashes }\end{array}$ \\
\hline & Sideswipe crashes & $\begin{array}{l}\text { Elimination of need to change lanes to avoid } \\
\text { delays behind a left-turning vehicle in the } \\
\text { inside through lane reduces sideswipe crashes }\end{array}$ \\
\hline & $\begin{array}{l}\text { Left-turn crashes due to offset } \\
\text { left turns }\end{array}$ & $\begin{array}{l}\text { Elimination of the negative offset between } \\
\text { opposing left-turn vehicles and increase of } \\
\text { available sight distance reduces left-turn } \\
\text { crashes }\end{array}$ \\
\hline & Bicycle and pedestrian crashes & $\begin{array}{l}\text { Bicycle lane separates bicycles from traffic; } \\
\text { pedestrians have shorter distance to cross and } \\
\text { can use a refuge area (if one provided) }\end{array}$ \\
\hline \multirow{3}{*}{ Other } & $\begin{array}{l}\text { Bicycle and pedestrian } \\
\text { accommodation due to lack of } \\
\text { facilities }\end{array}$ & $\begin{array}{l}\text { Opportunity to provide appropriate or required } \\
\text { facilities increasing use by such users }\end{array}$ \\
\hline & Aesthetics & $\begin{array}{l}\text { Provision for landscaped medians and other } \\
\text { treatments as see fit }\end{array}$ \\
\hline & Traffic calming & $\begin{array}{l}\text { Potential for uniform speeds and consistency; } \\
\text { opportunity to encourage pedestrian activity }\end{array}$ \\
\hline
\end{tabular}

Table 2 In-depth evaluation items

\begin{tabular}{|l|}
\hline Operational evaluation \\
\hline Travel times \\
\hline Intersection delays \\
\hline Travel speeds \\
\hline Minor street operations \\
\hline Railroad crossings \\
\hline Safety evaluation \\
\hline Angle crashes \\
\hline Left turn crashes \\
\hline Rear end crashes \\
\hline Pedestrian safety \\
\hline Bicyclists safety \\
\hline
\end{tabular}




\section{Operational Performance}

Road diets may be a feasible alternative on roadways with ADTs as high as 23,000 vehicles per day (vpd) but may also fail on roadways with low ADT (e.g., 5000, depending upon side street volume at major intersections. Figure 2 summarizes the range of operating parameters that would support a road diet project.

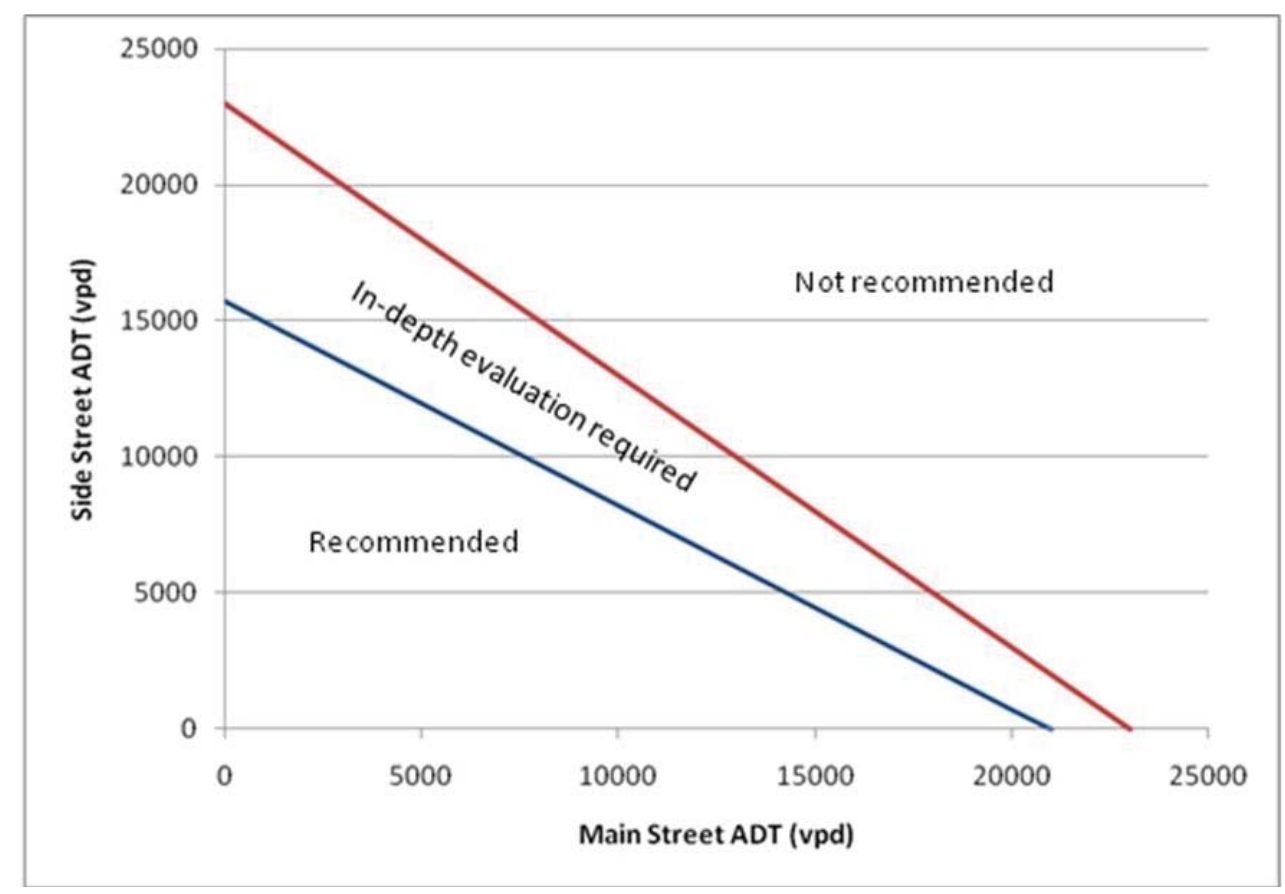

Figure 2 Operational performance guideline for road diet implementation

The red line in the figure identifies the volume combinations where the roadway will operate under capacity with a road diet. The blue line identifies the point where the operational performance, i.e. delays, is equal between a four-lane and three-lane cross-section. The combinations below the blue line indicate lower delays associated with the implementation of a road diet. Utilizing these concepts, Figure 2 defines three areas with respect to road diet installations. The lower area called "Recommended" identifies the volume combinations where the road-diet will perform below capacity and result in operational gains. The upper area "Notrecommended" identifies the volume combinations where the roadway will operate above capacity, and will have higher delays than the four-lane alternative. A road diet operating in this range will likely result in undesired congestion. The area between the two lines is considered as the area requiring additional investigation to determine the feasibility of a road diet conversion. A road diet in this area will operate under capacity, but may have higher delays than the fourlane section. Thus analysis should be completed to determine the impacts that would result from the road diet.

\section{Safety Performance}

Based on before and after analysis of case studies in Kentucky, road diets have demonstrated an average 36 percent reduction in total crashes compared to four-lane cross sections. National research has developed a crash modification factor of 0.71 based due to the introduction of a continuous left-turn lane on a roadway indicating a 29 percent reduction in crashes. While road diets can be effective in addressing crash problems, a safety analysis should be completed to 
determine the type of crashes on the corridor and ensure that they are correctable by the implementation of a road diet. The following crash types are common types that may be addressed by a road diet.

- Sideswipe Crashes. Sideswipe crashes can result from vehicles changing lanes to avoid delays behind a left-turning vehicle in the inside through lane. These types of crashes can occur at midblock access points and major intersections. Road diets eliminate these types of crashes by removing the turning vehicle from the through lane. Sideswipes can also occur between vehicles traveling on the two-way left-turn lane and those attempting to enter it but these crashes are not a frequent occurrence as prior research and case studies indicate. Figure 3 shows the region of decreased sideswipe conflicts, as a combination of major and minor street volumes and left-turn percentage.

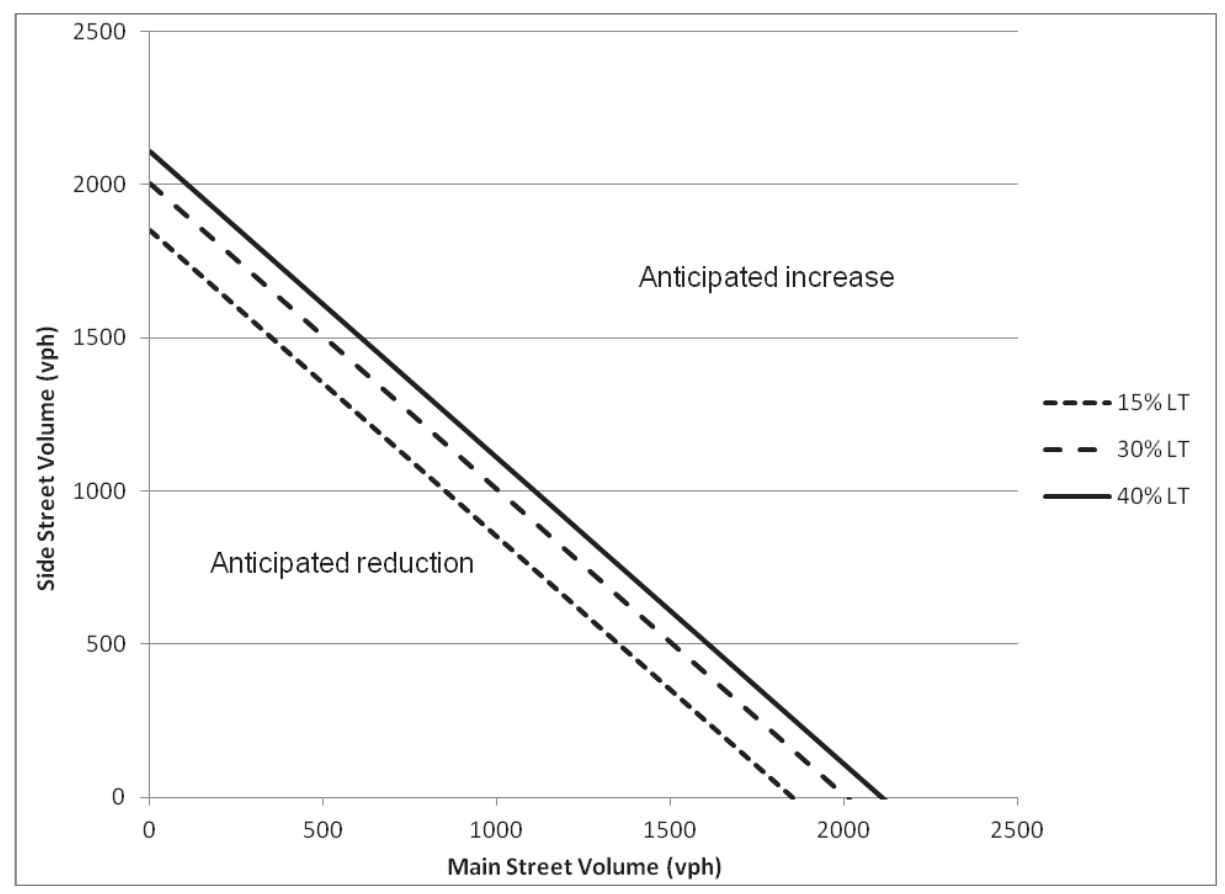

Figure 3 Sideswipe conflict guidance by major/minor street volume and left-turn percent

- Rear End Crashes. Rear end crashes can result from vehicles traveling in the inside through lane behind a stopping or stopped left-turning vehicle. A road diet reduces these types of crashes by removing the stopped turning vehicle from the through lane. Road diets are anticipated to reduce rear end crashes on roadways with high volumes of leftturn traffic; however, increased congestion resulting from the lane reduction may increase rear end crashes on the main street under other conditions. Figure 4 shows the region of decreased rear end conflicts, as a combination of major and minor street volumes and left-turn percentage. 


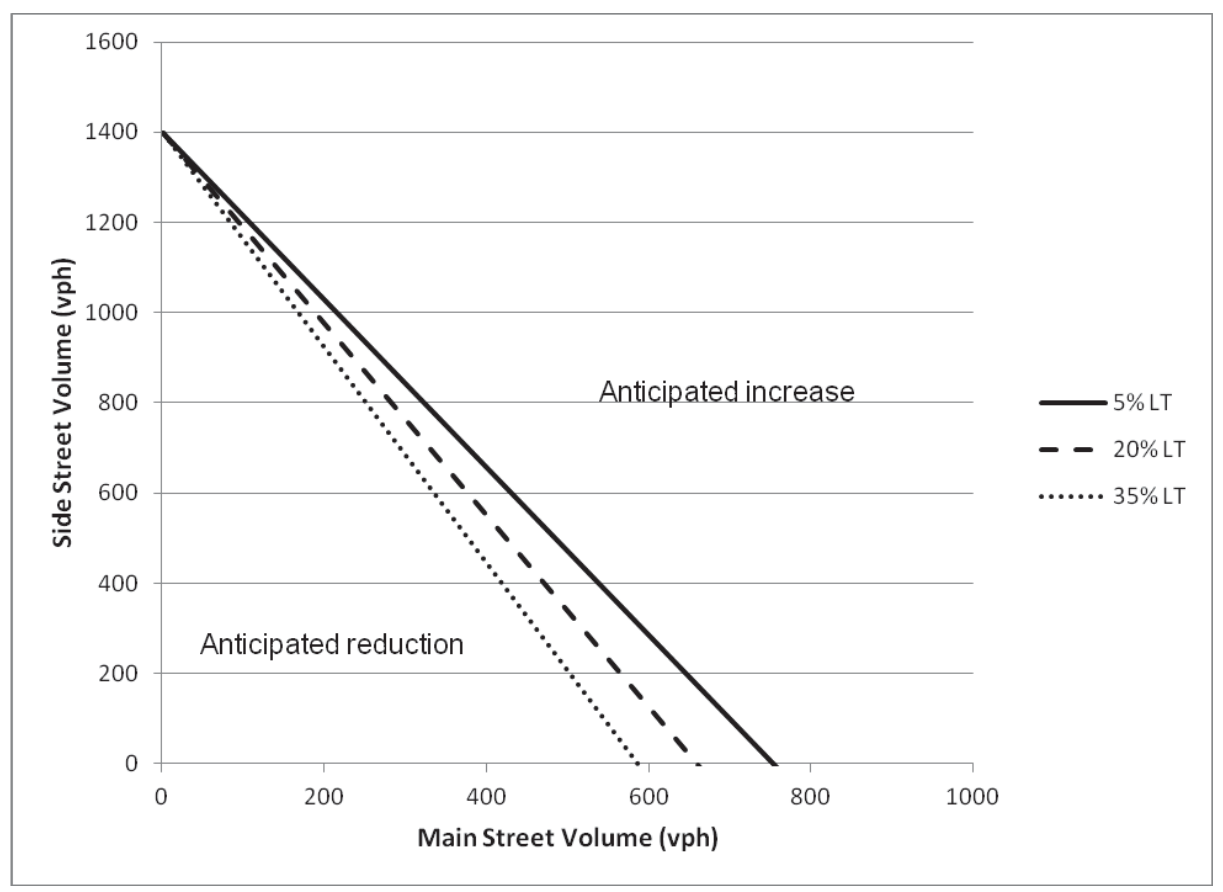

Figure 4 Rear end conflict guidance by major/minor street volume and left-turn percent

- Left-turn angle crashes. When moderate to high volumes of left-turn traffic exist on a roadway without dedicated left-turn lanes, a significant increase in crashes between major street left-turns and opposing through volumes can occur. This is due to the restricted sight distance that can occur when an opposing left-turn vehicle restricts sight distance to approaching through vehicles in the outside lane. Road diets address this type of crash by correcting the negative offset between opposing left-turn vehicles and significantly increasing available sight distance.

\section{Other Considerations}

There are additional elements that should be considered when road diets are evaluated. The following list identifies these elements and provides a brief overview of their potential implications.

- Multimodal operations: Road diets improve multimodal operations (bicycles and transit) through the presence of either wider lanes or exclusive bicycle lanes.

- Pedestrian safety: Road diets can increase pedestrian comfort and safety by reducing the number of lanes to be crossed, providing a refuge within the two-way left-turn lane and reducing the speed differential in the travel lanes

- Operational consistency: This aspect addresses the potential for more uniform speeds along the corridor, elimination of unbalanced speeds by travel lanes and potential speed reductions (road diet is acting as traffic calming) after the road diet conversion.

- Livability: Road diets have the potential to improve corridor livability and provide opportunities for residential and commercial growth. Reduced speeds encourage pedestrian and bicycle traffic and create a pedestrian friendly environment. 


\section{Design Considerations}

\section{Transition}

One of the primary considerations in the design of a road diet is the transition from a four-lane cross section to a three-lane cross section. This transition can serve as a capacity and safety concern if not designed properly. Ideally, a road diet would replace the entire four-lane section of roadway eliminating the need for a transition. However, if necessary the transition can be placed at either a major intersection or midblock.

If transitioning at a major intersection, the entering through lane should be dropped as a turn lane serving a significant volume of traffic, such as a heavy right turn movement. Dropping the lane as a right or left-turn lane for a minimal volume may be counterproductive as the weaving and merging operations associated with the lane drop occur simultaneously with the increased demands of the intersection. Figure 5 shows a lane drop as a right turn lane at an intersection.

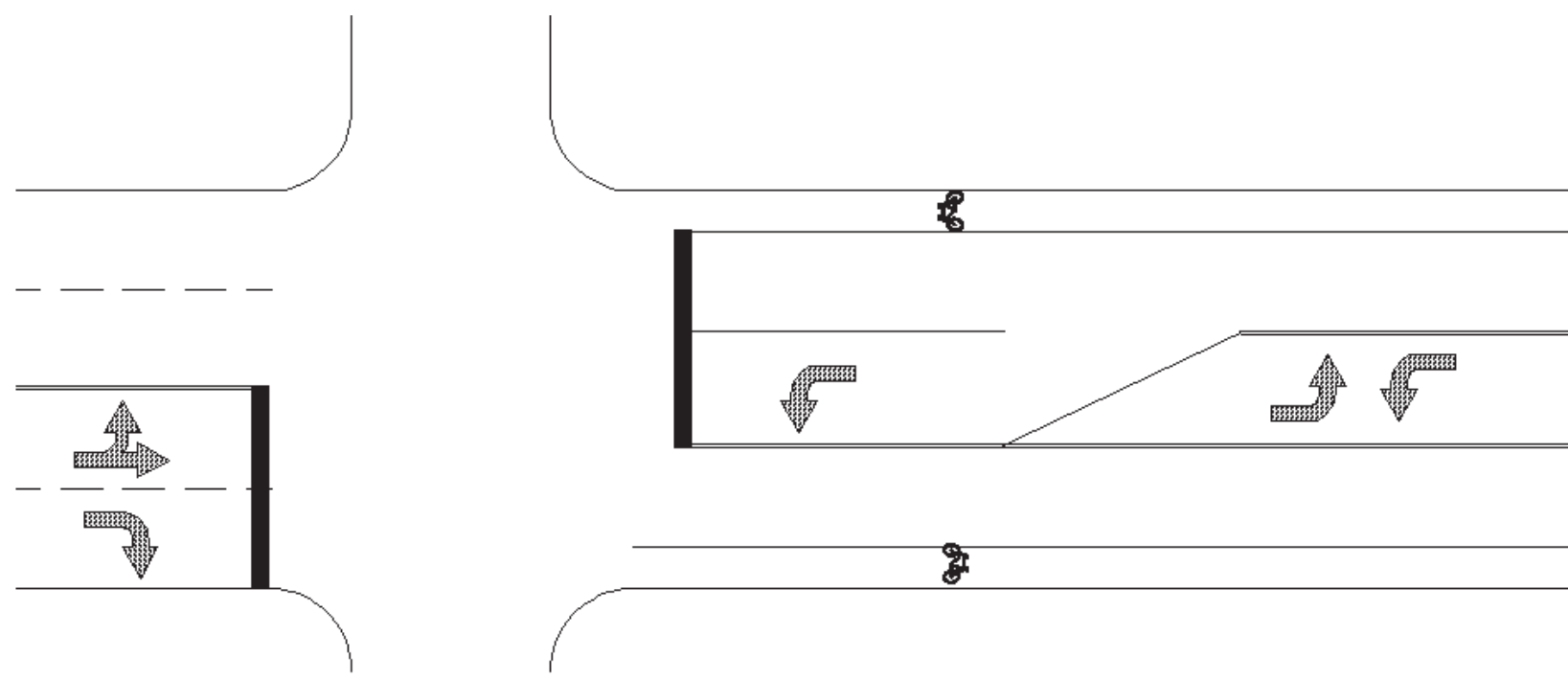

Figure 5 Right turn lane drop as road diet transition

A proper transition may also take place midblock on the roadway. When placing the transition midblock, it should occur away from intersections and/or high volume access points that would place stopped turning traffic in the through lanes of the merging traffic. Figure 6 shows a midblock transition.

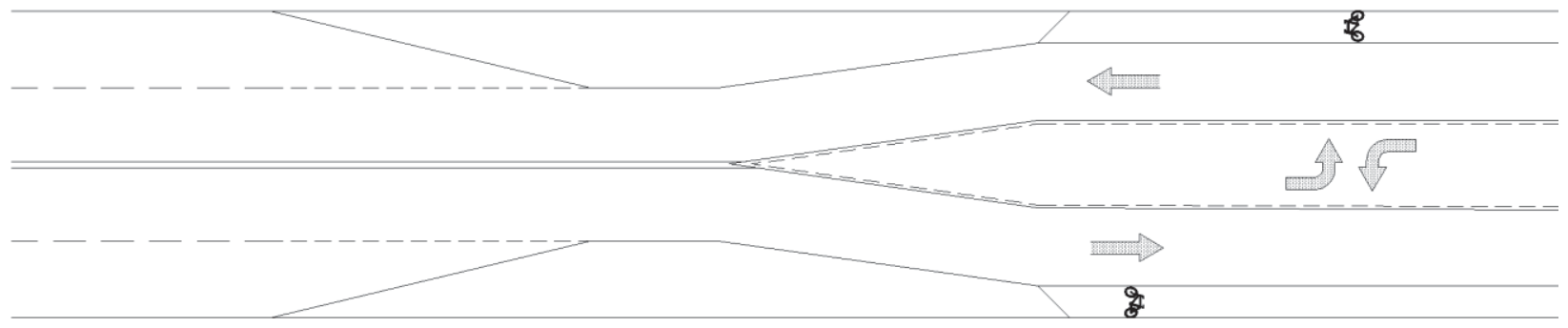

Figure 6 Midblock lane drop as road diet transition 
If not designed properly, these transitions can significantly increase crashes removing any safety benefit of the road diet. All transitions should follow AASHTO"s Policy on Geometric Design for Highways and Streets and MUTCD guidance for the reduction of through lanes.

\section{Cross Section}

The cross section of a four-lane roadway can vary widely from a 40 foot section with narrow lanes to a wide 60 foot section. The proposed cross section for the road diet should utilize the available space in the most efficient manner while properly channelizing intended movements. The following figures show possible configurations for road diet sections.

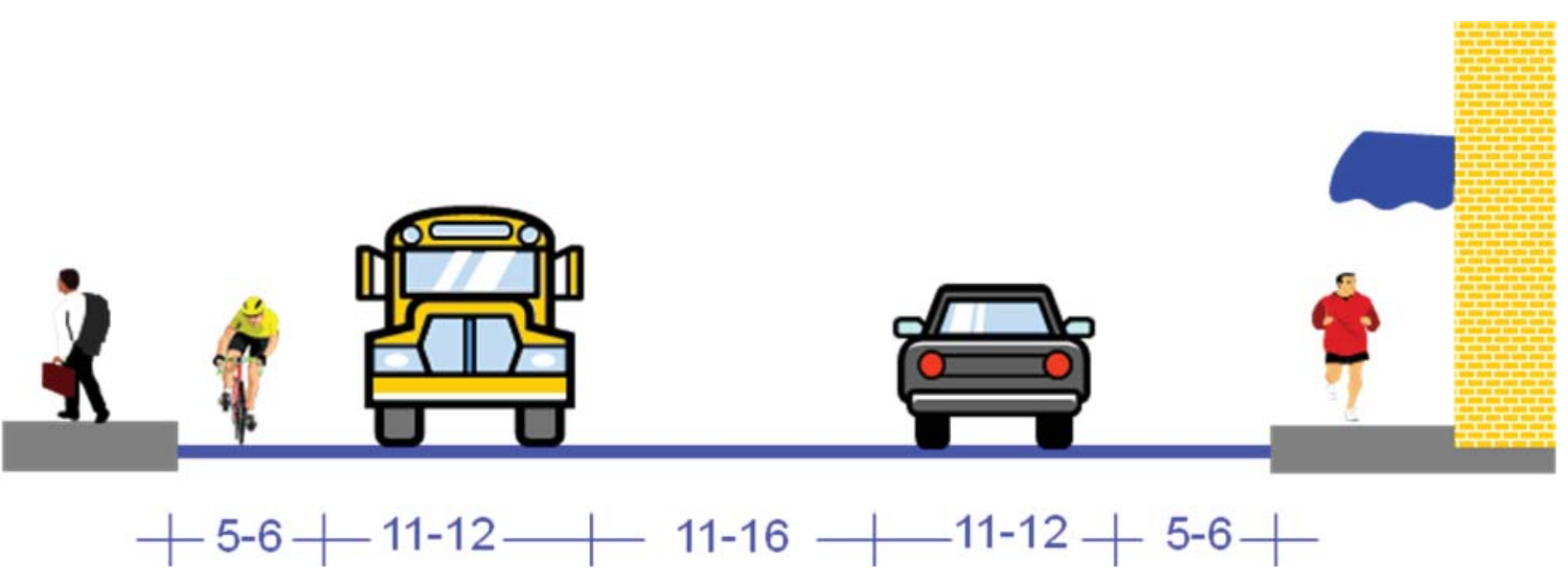

Figure 7 Example cross section with a two-way left-turn lane and bicycle lanes

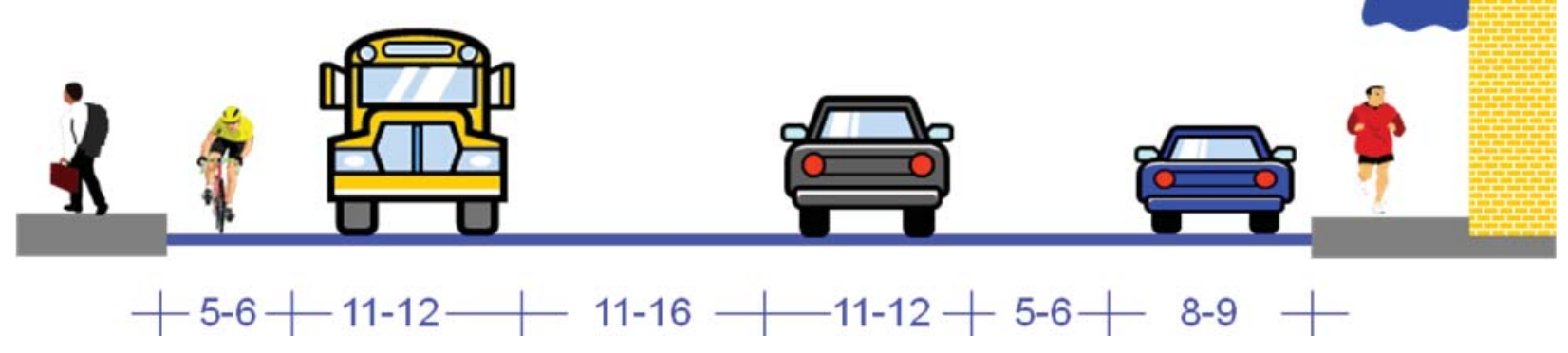

Figure 8 Example cross section with two-way left-turn lane, bicycle lanes and parking 


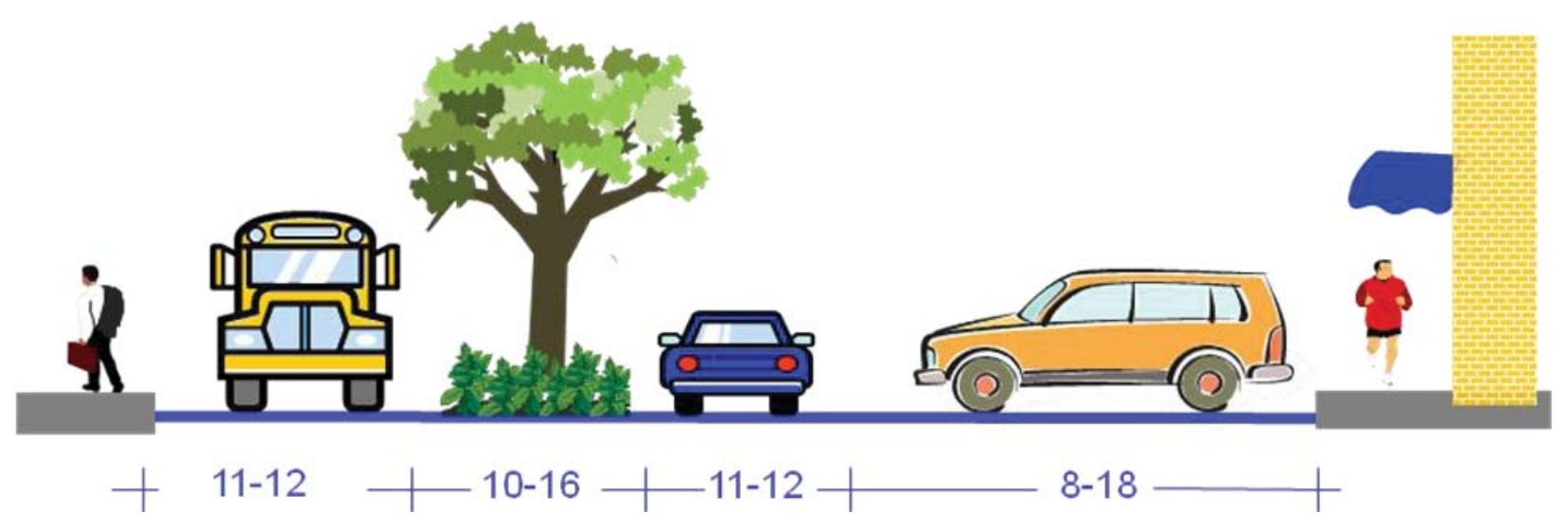

Figure 9 Example cross section with landscaped median and parking (back into shown)

\section{Access Density}

While access density can have a significant impact on angle and rear end crashes as vehicles slow to enter and exit an access point, no case studies have shown that this impact is greater on road diets than a four-lane cross-section. One area of concern for the operation of a twoway left turn lane is the potential for overlapping left-turn paths between the main street and adjacent access points. According to KTC Auxiliary Turn Lane Policy (2), a non-traversable median and turn restrictions are recommended when a corridor exceeds 85 access points per mile (based on number of access points on both sides of the street). Depending on the ingress and egress volumes of major access points, the four-lane section may outperform the three-lane section in some instances.

\section{Performance Evaluation}

Prior to the implementation of a road diet, it is recommended that a capacity analysis be completed for the major signalized intersections on the corridor to ensure that they would operate acceptably with a revised lane configuration. Analysis based on Highway Capacity Manual (HCM) methodologies is sufficient to check intersection capacities. For special cases, such as closely spaced intersections, coordinated signal systems, or corridors with at-grade rail crossings, micro-simulation is recommended to adequately evaluate arrival patterns and queue formation and dissipation.

\section{When Not to Convert}

There are cases where a road diet may not be the appropriate solution. These cases include scenarios where the road diet will not improve the operational efficiency of the corridor or corridors where at-grade rail crossing or other conditions, such as a high frequency of bus stops may create queues that require a long time to dissipate.

Road diets only address the specific crash types identified in the safety performance section. These crashes include rear end and sideswipes that result from an inside through lane that is blocked by left-turning traffic and left-turn angle crashes that result from offset left-turn lanes. If these crash types do not exist, a safety benefit would not be realized.

If an adequate transition cannot be designed for the road diet, it should be reconsidered, as this may cause an increase in crashes, and outweigh any derived benefit along the corridor. 
If left-turn lanes already exist on the corridor, even in limited locations at major intersections, the introduction of a road diet would adversely impact capacity and safety benefits may not be realized.

\section{Analysis Checklist}

The following checklist is provided in order to allow for a systematic consideration of the various elements to be evaluated and considered prior to a road diet implementation (Table 3). The checklist identifies analysis to be completed and data to be collected that may be used to assist in evaluating the road diet conversion for a specific section of roadway. Where applicable the guidance in this document may be used to address many of these issues.

Table 3 Checklist for implementation considerations

\begin{tabular}{|l|}
\hline Operational aspects \\
\hline Travel times \\
\hline Intersection delays \\
\hline Travel speeds \\
\hline Minor street operations \\
\hline Railroad crossings \\
\hline Safety aspects \\
\hline Angle crashes \\
\hline Left turn crashes \\
\hline Rear end crashes \\
\hline Pedestrian safety \\
\hline Bicyclists safety \\
\hline Multimodal operations \\
\hline Public transportation \\
\hline Bicycle \\
\hline Pedestrian \\
\hline Access management \\
\hline Livability \\
\hline Operational consistency \\
\hline Transition design \\
\hline Cross section elements \\
\hline Number of lanes \\
\hline Bicycle lanes \\
\hline Parking \\
\hline Sidewalks \\
\hline Buffers \\
\hline \\
\hline
\end{tabular}




\section{REFERNCES}

1. Stamatiadis, N., Kirk, A., Wang, C., and Cull, A. (2011) Guidelines for Road Diet Conversions, KTC-11-20, Kentucky Transportation Center, Lexington, KY.

2. Kentucky Transportation Cabinet (2009) Design Memo 03-09Manual, Frankfort, KY 
For more information or a complete publication list, contact us at:

\section{KENTUCKY TRANSPORTATION CENTER}

176 Raymond Building

University of Kentucky

Lexington, Kentucky 40506-0281

(859) 257-4513

(859) $257-1815$ (FAX)

1-800-432-0719

www.ktc.uky.edu

ktc@engr.uky.edu

The University of Kentucky is an Equal Opportunity Organization 